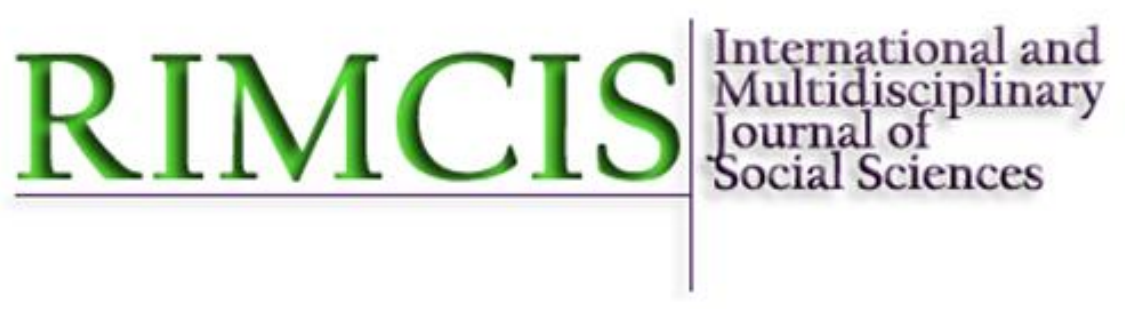

\title{
Volume 6, Number 2
}

\section{Hipatia Press}

www.hipatiapress.com

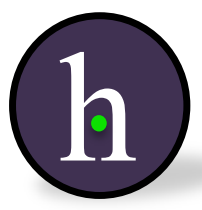

El Perfil del Votante del Cambio en España: De las Elecciones Europeas a las Generales del 20D - Irene Valero-Oteo.

Soluciones TIC para Personas Mayores: Preferencias Manifestadas en el Medio Rural Español - María Ángeles Rubio Pastor, Inmaculada Plaza García, Víctor Orive Serrano.

The Growth of E-Marketing in Business-to-Business Industry and its Effect on the Performance of Businesses in Pakistan: Marketing Success - Adnan Ahmed Sheikh, Arfan Shahzad, Awanis Ku Ishak...178

How Solidarity Influences Political Actors to Manage the Refugee Crisis: The Case of Proactiva Open Arms - Gemma Álvarez-Jiménez Maria Padrós-Cuxart

Reviews

Book Review: Cuerpos aliados y lucha política: Hacia una teoría performativa de la asamblea by Judth Butler - Teresa Plaja Viñas. 


\section{RIMCIS}

\section{Hipatia Press}

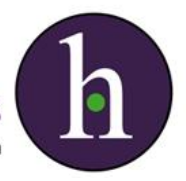

Instructions for authors, subscriptions and further details:

http://rimcis.hipatiapress.com

\section{El Perfil del Votante del Cambio en España: De las Elecciones Europeas a las Generales del 20D}

Irene Valero-Oteo ${ }^{1}$

1) Universidad del País Vasco - UPV/EHU, España

Date of publication: July $30^{\text {th }}, 2017$

Edition period: July 2017 - November 2017

To cite this article: Valero-Oteo, I. (2017). El Perfil del Votante del Cambio en España: De las Elecciones Europeas a las Generales del 20D. International and Multidisciplinary Journal of Social Sciences, 6(2), 107-136. doi: 10.17583/rimcis.2017.2395

To link this article: http://doi.org/10.17583/rimcis.2017.2395

\section{PLEASE SCROLL DOWN FOR ARTICLE}

The terms and conditions of use are related to the Open Journal System and to Creative Commons Attribution License (CC-BY). 


\section{Voters for the Change Profile in Spain: From European Elections to 20D General Elections}

Irene Valero-Oteo

Universidad del País Vasco (UPV/EHU)

\section{Abstract}

Global financial crisis, which became apparent on 2008, has had a devastating impact on social welfare of developed economies, including Spain. This state, combined with a sequence of another crisis, one of them institutional, that had remained dormant on Spanish society for decades, has raised doubts as to the ability of traditional politics to give solutions to population issues. In this context, Spain has witnessed deep changes in its political scene with the advent of two new political groups, which have made an strong entrance into the political territory and with the goal being to regenerate the politics of the country. This paper aims to analyze the profile of the voters who have chosen the new political groups, compared with the voters of traditional political groups, in order to observe differences and similarities between voters of new and old political groups.

Keywords: traditional political groups, new political groups, elections, change 


\section{Perfil del Votante del Cambio en España: De las Elecciones Europeas a las Generales del 20D}

Irene Valero-Oteo

Universidad del País Vasco (UPV/EHU)

\section{Resumen}

La crisis financiera mundial que se hizo visible en 2008 ha tenido consecuencias devastadoras para el bienestar social de las economías desarrolladas, entre ellas España. Esta situación sumada a otra serie de crisis, entre ellas institucional, que llevaban latentes en la sociedad española desde hace décadas, ha puesto en tela de juicio la capacidad de la política tradicional para dar solución a los problemas de la población. En este contexto, España ha visto transformado su escenario político con la irrupción en él de dos nuevas formaciones políticas, quienes han entrado con fuerza en el territorio político y cuyo objetivo es regenerar la política del país. En este trabajo lo que se intenta es analizar cuál ha sido el perfil de votante que ha apostado por las nuevas formaciones en comparación con los partidos tradicionales, con objeto de observar las diferencias y similitudes que existen entre los votantes de nuevos y viejos partidos políticos.

Palabras clave: partidos políticos tradicionales, nuevos partidos políticos, elecciones, cambio 


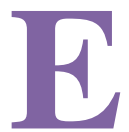

spaña lleva sumida en la inestabilidad desde el inicio efectivo de la crisis en 2008. Una inestabilidad, traducida en forma de crisis, que se ha manifestado en varias esferas de la vida del país: económica, política, institucional y social. Es verdad, que las instituciones españolas han estado anquilosadas desde su creación en la Transición, sin embargo, ha sido cuando ha estallado la burbuja inmobiliaria cuando se han destapado todas las ineficiencias institucionales de la política española que, además, se han visto deterioradas por los casos de corrupción (Sánchez-Cuenca, 2014). Es decir, los problemas que existen en el esqueleto institucional y político del país no han surgido de repente con la crisis, existen desde hace décadas, pero se han hecho más visibles y reconocibles para la población a partir de 2008, "pues una burbuja financiera no solo es un desastre económico, sino que también tiene un coste político considerable" (Galindo, Llaneras, Medina, San Miguel, Simón y Senserrich, 2015, 120). Todo ello ha generado que la sociedad española se haya convertido en una amalgama de ciudadanos que desconfían plenamente de sus instituciones. Las únicas instituciones que aprobaron la valoración de la sociedad española en 2015 fueron aquellas relacionadas con las fuerzas y cuerpos de seguridad del Estado (Guardia Civil, Fuerzas Armadas, policía) ${ }^{1}$. El resto de instituciones no superó la calificación de cinco (en una escala de 0 a 10), siendo los partidos políticos $(2,33)$, los sindicatos $(2,61)$ y el gobierno $(2,77)$, las instituciones peor valoradas $^{2}$. Esta desconfianza, como se señalaba antes, se ha disparado con los casos de corrupción y la mediatización de los mismos, pues la mayoría de la población en España cree que la gente que desconfía de los políticos lo hace debido a la corrupción (CIS, 2012; estudio 2930). Sin embargo, a esta corrupción se añade la respuesta política de los gobiernos en España ante la crisis, basada en medidas de austeridad, que no han conseguido mejorar la situación de la población en España, sino que han favorecido el aumento de las desigualdades sociales en el país, lo que ha dado lugar a la percepción, por parte de la ciudadanía, de la incapacidad de los gobiernos para dar respuesta a sus problemas cotidianos (Estefanía et al., 2015). De esta manera, la crisis, la desconfianza política y la incapacidad de los partidos políticos percibida por los ciudadanos, han generado un descontento social de la ciudadanía hacia los partidos políticos tradicionales (Laparra y Pérez Eransus, 2012), incluso, podría halarse de crisis de los partidos tradicionales. 


\section{Valero-Oteo - El Perfil del Votante del Cambio en España}

Todo lo anterior ha facilitado la aparición de nuevas formaciones políticas que abogan por un cambio en la forma de hacer política en España. Desde las elecciones al Parlamento Europeo de 2014 dos nuevos partidos políticos han irrumpido en la escena política española: Podemos y Ciudadanos (C's), si bien este último se hizo visible de forma más tardía, a partir de 2015. Estas formaciones han ido ganando progresivamente terreno a PP y PSOE, de manera que se puede hablar de la ruptura del bipartidismo tradicional en la historia de la democracia en España. Si en 2011 PP y PSOE se repartían el 84,5\% de los escaños del congreso (186 escaños el PP y 110 el PSOE) y el $73,39 \%$ de los votos (44,63\% el PP y $28,76 \%$ el PSOE) en 2015 la suma de los dos partidos ha descendido a un 60,8\% de los escaños y un $50,73 \%$ de los votos, pues las dos nuevas formaciones han absorbido un $31,1 \%$ de los escaños y un $34,59 \%$ de los votos ${ }^{3}$.

Este trabajo se centrará en analizar las características de los votantes de las nuevas formaciones políticas, es decir, de las personas que han escogido el cambio, en contra posición a los votantes de los partidos establecidos, los partidarios del inmovilismo. En este sentido, el trabajo quiere dar cuenta de las diferencias que existen entre los votantes de los nuevos y viejos partidos y las diferencias entre los votantes de las nuevas formaciones políticas.

\section{Metodología}

El estudio del comportamiento electoral es una rama consolidada de estudio que se mueve a caballo entre la Ciencia Política y la Sociología y que ha cobrado en los tiempos actuales de transformación una relevancia especial. El procedimiento más habitual para su estudio es el análisis de series bien de la intención, bien del recuerdo de voto ejercido en las distintas convocatorias electorales, tal como son recogidas en las encuestas pre- y post-electorales. Generalmente, esta intención o recuerdo de voto expresado por los entrevistados en la encuesta es objeto de reelaboración para mitigar el efecto distorsionador que la "opinión pública" ejerce sobre las respuestas demoscópicas, a través de diversos efectos como la "subida al carro ganador", que sobrestima a los ganadores de las elecciones (Cabrera, 2014) o el ocultamiento de las posiciones a priori minoritarias ("espiral del silencio" en la terminología de Noelle-Neumann, 2010) que generalmente se 
convierte en abstención ficticia. Esto es lo que se conoce como "cocina", de la que se ocupan los institutos de opinión con mayor o menor éxito predictivo, no exento en ocasiones de polémica. En cualquier caso, a pesar de algunos de los sesgos existentes, cabe destacar la utilidad que presentan los datos de este tipo de encuestas a la hora de predecir y explicar los cambios políticos que ocurren antes y después de los comicios electorales. Los mencionados datos de "cocina" son los que suelen llegar a la opinión pública y que pretenden reflejar de una manera lo más realista posible la distribución efectiva del voto en los comicios. Sin embargo, este "voto estimado", no es el objeto del presente trabajo, sino lo que en el argot técnico se conoce como "voto directo", esto es, las respuestas directamente obtenidas a las preguntas del cuestionario sin proceso estadístico posterior. De esta manera, a partir de los microdatos de las diferentes encuestas, se pueden explorar en profundidad los flujos entre las distintas opciones de voto tal cual son expresados por los entrevistados.

El objetivo de este trabajo es analizar el perfil ciudadano que ha decidido apostar por las nuevas fuerzas políticas, quienes han irrumpido en el panorama político dominante de los últimos dos años, esto es, Podemos y Ciudadanos. Para llevar a cabo esta tarea analítica se tomarán las series de datos de recuerdo "directo" de voto, construidas a partir de las encuestas post- electorales del Centro de Investigaciones Sociológicas (CIS), el más prestigioso centro de estudio de opinión pública del país. Estas encuestas suelen contar con unos requerimientos técnicos de alta calidad, lo que las sitúa como la fuente más reputada entre los estudiosos del comportamiento electoral.

Este trabajo se trata de una investigación empírico-descriptiva, en la que se compara el perfil de los votantes de los nuevos partidos (Podemos y C's) con el de los partidos hegemónicos españoles, PP y PSOE, a la vez que se comparan los perfiles de cada uno de los nuevos partidos. De esta manera, se extrae la información necesaria para evidenciar que el electorado de Podemos y C's se diferencia de los votantes de los partidos tradicionales y que, a su vez, existen diferencias entre los votantes de ambos partidos. Es decir, lo que se pretende es averiguar si existen (y si es así, cuáles son) características específicas entre los votantes del cambio, que dan lugar a la creación de un perfil específico, cuando se las compara con las de los 


\section{Valero-Oteo - El Perfil del Votante del Cambio en España}

partidos tradicionales que han protagonizado el escenario político en las últimas décadas. Además, se trata de averiguar si dentro de su igualdad, en contraposición a PP y PSOE, existen diferencias entre los votantes de C's y Podemos.

Lo que se pretende investigar es la incidencia que tienen ciertos factores sociales e individuales sobre el voto a Podemos y a Ciudadanos. Para ello, se van a calcular porcentajes de columna ${ }^{4}$ mediante tablas de contingencia, obtenidas a través de cruces entre una variable dependiente, relacionada con el recuerdo de voto con variables de tipo sociodemográfico, sociopolítico y socioeconómico.

- Recuerdo de voto: “¿Podría decirme a qué partido o coalición votó en las pasadas elecciones?

Asimismo, como variables independientes se han escogido varias de las preguntas de los cuestionarios que aportan una valiosa información a la hora de votar a los nuevos y viejos partidos:

1. Variables sociodemográficas: nivel de estudios, edad, tamaño de municipio y estatus socioeconómico.

2. Variables políticas: autoubicación ideológica, valoración sobre la situación política de España, recuerdo de voto en las elecciones de 2011, participación política e interés en la campaña electoral.

3. Variables económicas: valoración de la situación económica personal, ingresos económicos mensuales y situación laboral.

El método de análisis predominante en el estudio del perfil del votante del cambio va a ser la tabla de contingencia. Las tablas de contingencia sirven para estudiar, inicialmente, la asociación entre variables y, por extensión, el efecto (mediante la dependencia de valores) de una o varias variables sobre otra y son, posiblemente, el método estadístico más ampliamente utilizado, al menos en estudios de corte sociológico (Escobar Mercado, Fernández Macías y Bernardi, 2010, 231-232).

En definitiva a través de la elaboración de tablas de contingencia se pretende obtener resultados que permitan establecer cuáles son las características (variables) que marcan la diferencia entre los electores de los 
nuevos y los viejos partidos, así como las características (si existieran) que diferencian a los votantes de Podemos y C's.

\section{El Perfil del Votante del Cambio}

\section{Perfil Sociodemográfico}

En este apartado el objetivo es averiguar cuáles son las características sociodemográficas de la población que diferencian a unos votantes de otros, además de comparar la evolución del perfil sociodemográfico de los votantes desde las elecciones al PE, concretamente, el de los votantes de las nuevas fuerzas políticas.

De acuerdo con el tamaño de municipio en el que residen los votantes (Tabla 1, anexos) en las zonas rurales (menores o iguales a 10.000 habitantes) la presencia de los partidos tradicionales es mucho más acusada, el $38,6 \%$ y el $35,9 \%$ de los votantes que viven en zonas rurales manifestaron su voto a PP y PSOE, respectivamente, en las elecciones del 20 de diciembre de 2015 , frente a un $12,4 \%$ y un $13,1 \%$, a C's y Podemos. De la misma manera, los mayores porcentajes de voto entre los electores de estos dos últimos partidos, se encuentran entre la población de las zonas más pobladas, es decir, municipios superiores a los 100.000 habitantes. Esto refleja el carácter más urbanita de los votantes tanto de Podemos, como de C's. Esto no quiere decir que en las zonas rurales no se vote a los nuevos partidos y en las zonas urbanas no se vote a los tradicionales, los votos se reparten por toda la geografía de España; pero sí quiere decir que en el mundo rural se tiende a votar más a las dos grandes coaliciones tradicionales con respecto a las zonas más pobladas. Si tenemos en cuenta los intensos desequilibrios representativos de la ley electoral (d'Hondt), que favorece a los territorios menos poblados y más rurales (Lago, 1999), potenciando el voto a los partidos tradicionales; destaca, aún más, el éxito en la consecución de escaños por parte de C's y Podemos, quienes han logrado lo que otros partidos minoritarios no habían conseguido hasta ahora: lograr un amplio número de escaños partiendo de una clara situación de desventaja electoral.

En comparación con los datos de las elecciones europeas de 2014, el resultado es el mismo. Los votantes de las dos fuerzas mayoritarias fueron 


\section{Valero-Oteo - El Perfil del Votante del Cambio en España}

más numerosos en el mundo rural, mientras que la mayoría de los votantes de los nuevos partidos se encontraban en las grades zonas urbanas. Cabe destacar los bajos porcentajes de voto a C's que aparecen en esta gráfica (fenómeno que se repetirá en las posteriores tablas referidas a las pasadas elecciones europeas), lo que se debe al menor conocimiento de dicho partido en ese período, pues, como se señalaba anteriormente, su presencia mediática ha sido más acusada a partir del año 2015.

La edad suele ser un factor decisivo a la hora de votar a un partido u otro, puesto que los jóvenes son actores con mayor propensión al cambio electoral y los mayores son los que presentan mayor aversión al cambio y a los riesgos que dicho cambio pueda entrañar (González y Caínzos, 2012). En este sentido, cuanto mayor sea el cambio que se pueda dar en el juego electoral, mayor es el papel que se adjudica a los jóvenes en el mismo (Salido y Martín, 2007). Así pues, en este análisis se aprecia cómo la edad influye en la tendencia de voto a cada uno de los partidos. En la Figura 1 se observa cómo va aumentando el voto a PP y PSOE conforme va aumentando la edad de los encuestados. Si bien la tabla muestra cómo el PP es un partido más envejecido que el PSOE, pues más de la mitad de los votantes (un $54,3 \%$ ) mayores de 65 recuerdan haber votado al PP en las elecciones del 20D, mientras que el PSOE reparte más sus votos entre los mayores de 65 $(32,2 \%)$ y las personas de entre 50 y 64 años $(37,8 \%)$. Entre las dos nuevas fuerzas políticas ocurre justamente lo contrario: a medida que disminuye la edad de los votantes aumenta el porcentaje de recuerdo de voto a estos dos partidos. De esta manera, el 59,4\% de los votantes que se encontraban en la franja de edad entre los 18 y los 36 años, manifestaron haber votado a Podemos o C's en las generales de 2015. Bien es verdad, que Podemos se muestra como el partido preferido de los más jóvenes, pues el porcentaje más alto del voto de los jóvenes fue a Podemos con un 32,7\%. Por su parte, C's es, también, un partido marcadamente joven pero reparte sus votos, de manera más equitativa que Podemos, entre los votantes de 18 a 36 años $(26,7 \%)$ y de 37 a 49 años $(25,5 \%)$. 


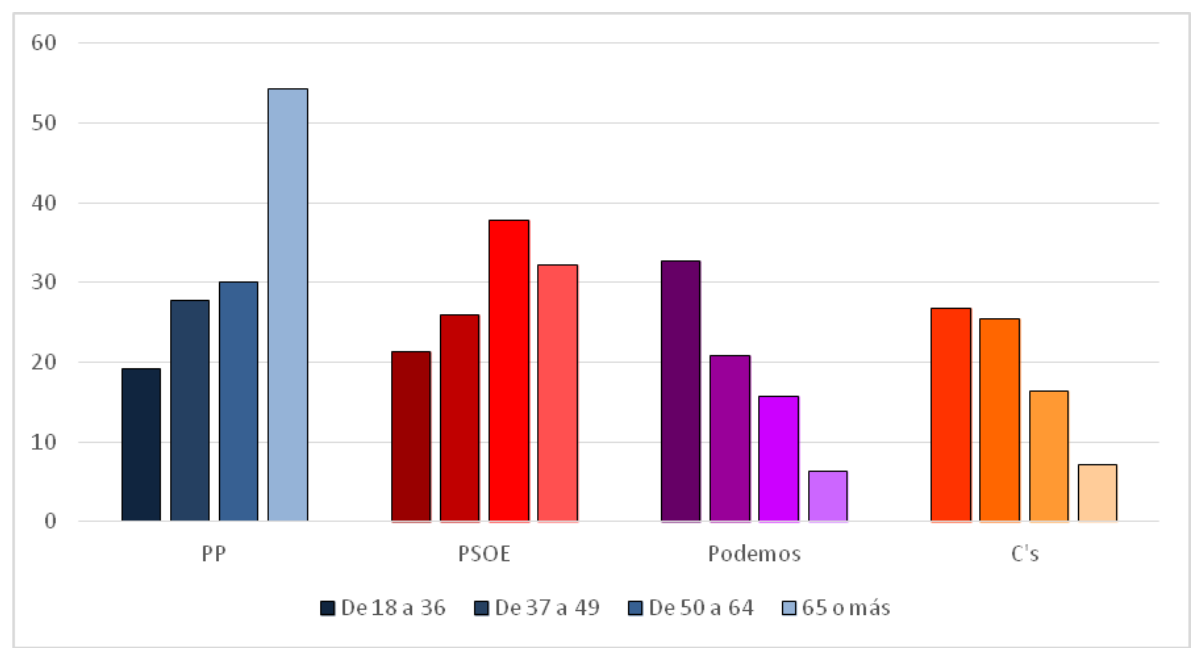

Figura 1. Intención de voto*Edad (2016)

Fuente: Elaboración propia a partir de los datos del CIS. Estudio no 3126

En cuanto a las elecciones al PE de 2014, los datos son similares. Los partidos tradicionales eran, después de las elecciones europeas, los partidos más envejecidos, igual que ahora. Sin embargo, en cuanto a los dos nuevos partidos, Podemos se mostraba en 2014 más joven que a finales de 2015, al revés que C's, que en 2014 tenía un porcentaje de votos en la franja de edad de 37 a 49 años mucho mayor que en el resto de intervalos de edad.

Si se compara la edad media del voto a los partidos en los dos períodos las diferencias se observan con mayor claridad (Figura 2). Lo más destacable es la disminución en la edad media de voto a C's, de 47,74 años en 2014 a 43,66 años en 2015, lo que quiere decir que en año y medio este partido ha sido capaz de atraer el voto de muchos jóvenes a su partido. Por el contrario Podemos, ha envejecido desde las europeas, aunque de forma menos acusada con un año de variación en la media, lo que significa que ha ampliado su abanico de votantes a gente más mayor. 


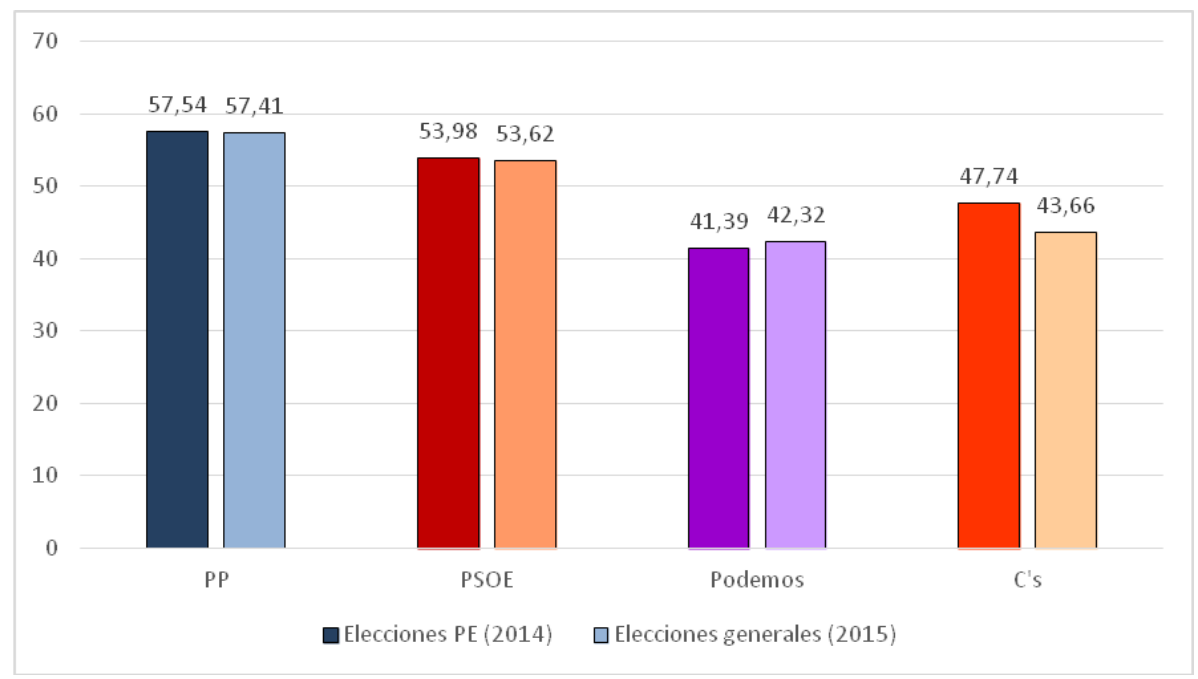

Figura 2. Evolución de la edad media de voto a los partidos.

Fuente: Elaboración propia a partir de los datos del CIS. Estudios 3028, 3126.

El nivel de estudios de la población, también influye marcadamente en el voto a un partido u otro. Los partidos tradicionales presentan una mayoría de votantes con niveles de estudios primarios o inferiores, tanto es así que el $85 \%$ de los votantes con estos niveles de estudios manifestaron haber votado al PP $(43,1 \%)$ o al PSOE $(41,9 \%)$ en las pasadas elecciones de diciembre (Tabla 2, anexos). Por el contrario Podemos y C's presentan un perfil de votante mayoritario con estudios superiores. Los votantes de los nuevos partidos se caracterizan, pues, por poseer un perfil con un nivel de estudios superior al de los votantes de los partidos tradicionales. Sin embargo, cabe mencionar que dentro del perfil de los nuevos partidos, C's es el que presenta más votantes con estudios universitarios, un $28,7 \%$, frente a Podemos con un $25,7 \%$.

En comparación con las elecciones al PE, el panorama ha cambiado ligeramente. En las elecciones europeas de 2014 los partidos que tenían mayor porcentaje de votantes con estudios universitarios eran PP y C's, frente al PSOE, donde predominaban los votantes con estudios primarios o menos y Podemos, con un perfil mayoritario de votante con niveles de 
estudios secundarios, aunque el número de universitarios también era considerable.

Para finalizar el perfil sociodemográfico del votante del cambio, hay que destacar la importancia de la variable estatus socioeconómico ${ }^{5}$ a la hora de elegir la papeleta del voto. En este caso concreto, los perfiles de los votantes de PP y PSOE se separan y dejan de converger como lo hacían respecto a las variables anteriores. El PSOE encuentra a sus mayores votantes entre los obreros (cualificados y no cualificados), mientras que el PP lo hace entre las viejas clases medias, pues casi la mitad de las personas que pertenecen a dicho grupo (49,3\%), manifestó haber votado al PP (Figura 3). Por su parte, Podemos presenta un perfil mayoritario de votantes de clase alta/media alta, al igual que C's quien también presenta altas cotas de voto en este grupo, aunque su perfil mayoritario es de nuevas clases medias.

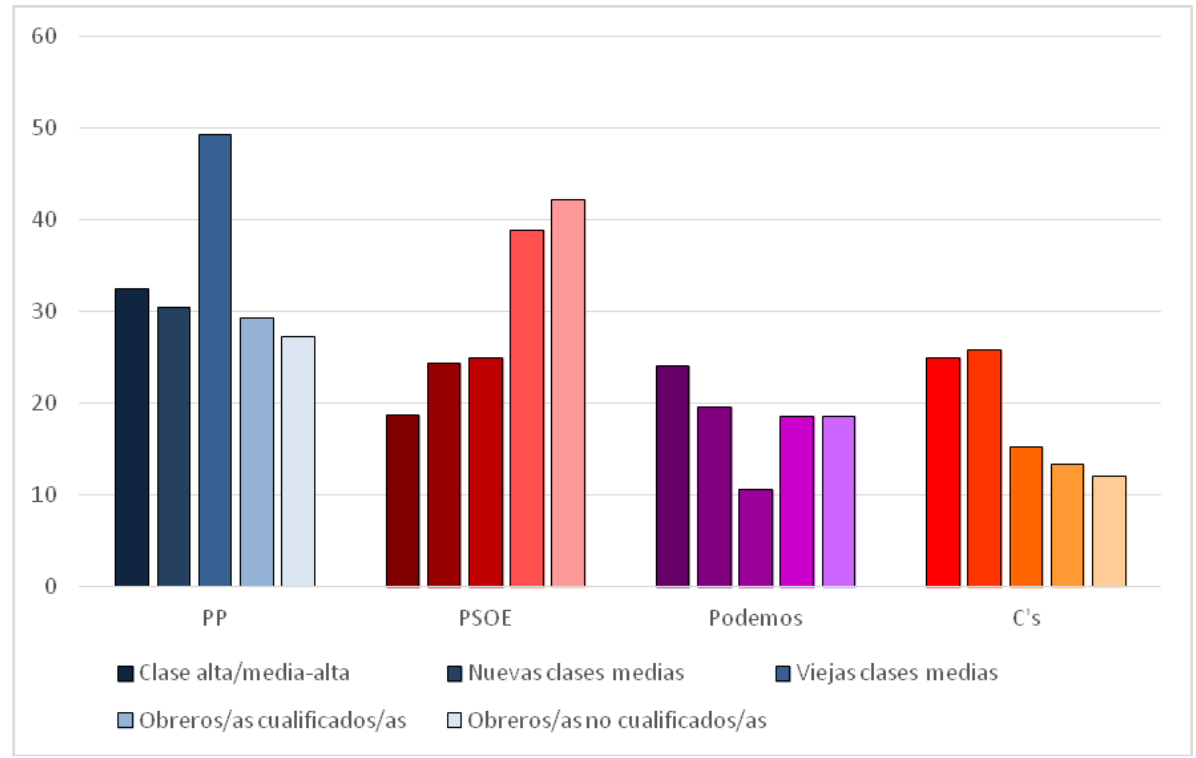

Figura 3. Recuerdo de voto (2015)*Estatus socioeconómico (2016)

Fuente: Elaboración propia a partir de los datos del CIS. Estudio n ${ }^{\circ} 3126$

En relación a las elecciones europeas de 2014, los resultados de los perfiles de voto son similares. Los datos más destacables son el mayor 


\section{Valero-Oteo - El Perfil del Votante del Cambio en España}

número de votantes de las nuevas clases medias en Podemos, que superaban a la clase alta y media alta en 2014, al contrario que en C's, donde para 2014 predominaba esta última clase, por encima de las nuevas clases medias.

Hasta ahora se han podido comprobar numerosas diferencias entre los votantes de los denominados viejos y nuevos partidos políticos. Sin embargo las diferencias entre los votantes de estos dos nuevos partidos son mínimas, por lo tanto, el perfil sociodemográfico del cambio está bastante unificado. En las siguientes páginas comprobaremos si las similitudes entre los votantes de estos nuevos partidos también existen en el plano político y económico.

\section{Perfil Sociopolítico}

En primer lugar es interesante analizar cuál es el perfil ideológico de los votantes de cada uno de los partidos y es en este punto donde quizá se encuentren las mayores diferencias entre los dos nuevos partidos. La autoubicación ideológica de los electores, marca un claro sesgo a la hora de votar a cada uno de los cuatro partidos mayoritarios (Tabla 3, anexos). El PP es el partido político por el que se decanta la mayor parte de los votantes con un perfil de ideología de extrema derecha, derecha y, en menor medida, de centro. C's, por su parte, ha sido elegido como el partido favorito de los electores del centro del espectro ideológico, ganándole terreno en este sector al Partido Popular, aunque una pequeña parte $(13,2 \%)$ de los electores que se autodefinen de derechas, manifestó también haber votado a Ciudadanos. Al otro lado del eje, en la izquierda política, se ubican los electores de Podemos y PSOE. El primero, es el partido elegido, sobre todo, por las personas que se definen como partidarias de la extrema izquierda $(58,1 \%)$, mientras que el PSOE estaría más concentrado en una izquierda más moderada $(59,2 \%)$.

En relación a las elecciones europeas se puede señalar que los resultados son parecidos. Podemos mantiene su carácter ideológico desde 2014, aunque en 2015 se ha radicalizado ligeramente. Por su parte, C's ha ido ganando terreno tanto en el centro del espectro ideológico, como se señalaba anteriormente, como a la izquierda y, sobre todo, a la derecha del mismo, pero siempre en el sector moderado. Comparando la media ideológica de los partidos ${ }^{6}$, para cada período, se ve de manera más resumida lo expuesto anteriormente (Tabla 1). 
Tabla 1

Comparación de la media ideológica de los partidos

\begin{tabular}{lll}
\hline & 2014 & 2015 \\
\hline PP & 7,04 & 6,95 \\
PSOE & 3,72 & 3,68 \\
Podemos & 3,29 & 3,15 \\
C's & 5,07 & 5,47 \\
\hline
\end{tabular}

Fuente: Elaboración propia a partir de los datos del CIS. Estudios n $3028, n^{\circ} 3126$.

Una vez visto el perfil ideológico de los votantes de los nuevos y viejos partidos, es interesante analizar a qué partidos votaron con anterioridad (en las elecciones de 2011) los nuevos votantes de Podemos y C's, es decir, a qué partidos han "robado" los votos las nuevas formaciones políticas. De esta manera, la Figura 4 muestra cómo casi el $60 \%$ de los votos de Podemos en 2015 proceden de los partidos PSOE e IU y en el caso de C's, de PP y PSOE. De esta manera se corrobora, con los análisis anteriores, que Podemos ha arrastrado a sus filas a los votantes de izquierda y extrema izquierda y C's a los de centro derecha y, en menor medida, centro izquierda. Sin embargo, ambos partidos presentan un alto porcentaje de personas que no votaron, votaron nulo, en blanco, no contestaron o no recuerdan a quien votaron en 2011 (28\% en Podemos y 26,7\% en C's).

La situación en 2014 era algo diferente. En el caso de Podemos éste partido era más variopinto, recogía mayor porcentaje de voto a partidos como PP o UPyD o a otros partidos, aunque predominaba el voto a PSOE e IU (64,4\%), en mayor medida que en 2015 (Figura 5). De la misma manera, el porcentaje de voto nulo, en blanco, abstención, no sabe o no recuerda, era mucho menor en 2014 que en 2015 (13,1\% frente a 28\%). C's, por su parte, ha cambiado su estructura de voto. Desde las europeas, C's ha absorbido a un amplio número de votantes de PP y PSOE: en 2014 los porcentajes eran de $34,4 \%$ y $9,4 \%$, respectivamente; mientras que en 2015 éstos se han convertido en un $41,4 \%$ y un $19,1 \%$. Esto corrobora que C's se ha hecho hueco tanto en el centro del espectro político, como a la derecha y a la izquierda del mismo, como se señalaba anteriormente. Por otro lado, en 2014 el 53,8\% de los votantes de C's, votaron en 2011 a "otros partidos" o había 
votado blanco, nulo, no se acordaban o no contestaron. Lo que se puede extraer de esta comparación del trasvase de votos a Podemos y C's entre los años 2014 y 2015 , en relación al voto en las elecciones de 2011, es que los nuevos partidos, han atraído a un gran número de votantes de los partidos más relevantes y que en las elecciones de diciembre, atrajeron el voto de personas que anteriormente habían decido votar en blanco o nulo, ya fuera por una actitud de duda frente al voto, desinterés, indiferencia o descontento con el abanico de partidos que se ofrecía en el año 2011.

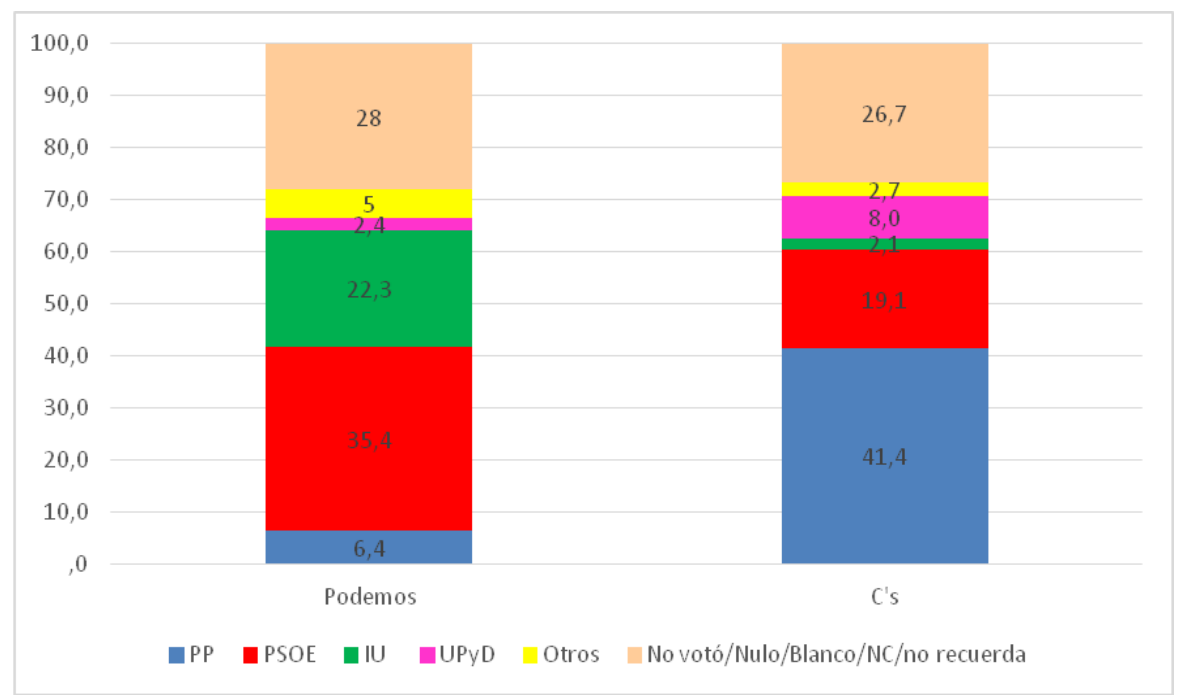

Figura 4: Recuerdo de voto en las elecciones de 2011 (2015)

Fuente: Elaboración propia a partir de los datos del CIS. Estudio 3126 


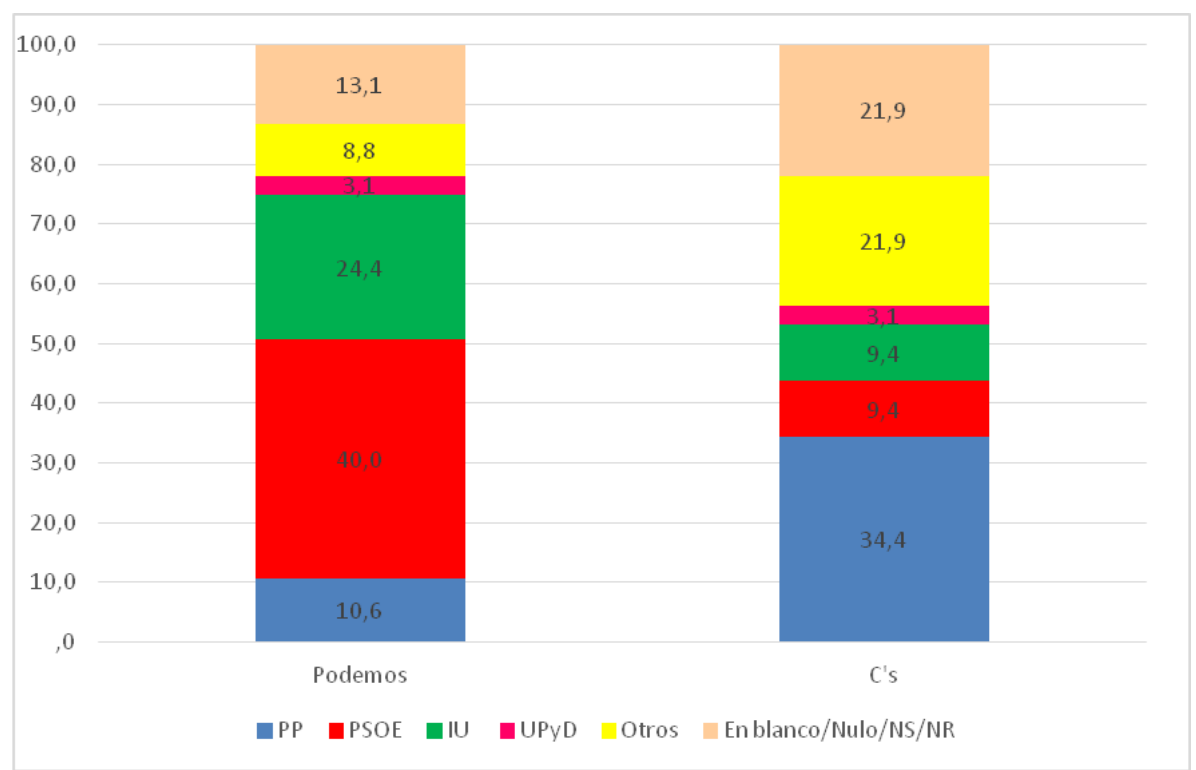

Figura 5: Recuerdo de voto en las elecciones de 2011 (2014)

Fuente: Elaboración propia a partir de los datos del CIS. Estudio no 3028.

Las siguientes variables a analizar en este apartado están relacionadas con las actitudes de los votantes hacia la política, concretamente, se van a analizar variables como el grado de interés con el que los votantes han seguido la campaña electoral, la valoración que hacen de la situación política del país y el tipo de participación política en la que más se implican los votantes de los diferentes partidos. El primer análisis consiste en observar si el interés con el que los votantes siguieron la campaña electoral del 20D está relacionado con el voto a los partidos. Los nuevos partidos presentan un perfil mayoritario de votantes que han seguido la campaña electoral con mucho interés, en especial Podemos. Por el contrario, tanto PP como PSOE, se caracterizan por tener un perfil de votante muy poco interesado en el transcurso de la campaña electoral, sobre todo, dentro del partido del PSOE, pues el 75,1\% de los/as encuestados/as que siguieron la campaña con ningún interés, manifestó haber votado a los dos partidos tradicionales (Tabla 4, anexos). 
En cuanto a la valoración acerca de la situación política por la que pasa el país, a excepción del PP, quien presenta una mayoría de votantes que califican la situación de manera positiva, el resto de partidos presenta un perfil de voto crítico con la situación política, valorándola como mala o muy mala.

Tabla 2

Participación política de los votantes de los diferentes partidos

\begin{tabular}{|c|c|c|c|c|c|}
\hline Frecuencia & $\begin{array}{l}\text { Asistir a una } \\
\text { manifestación }\end{array}$ & $\begin{array}{l}\text { Boicot a } \\
\text { productos }\end{array}$ & $\begin{array}{l}\text { Participar en } \\
\text { una huelga }\end{array}$ & $\begin{array}{c}\text { Ocupar/ } \\
\text { encierros/ } \\
\text { bloqueo tráfico }\end{array}$ & $\begin{array}{c}\text { Participar en sitios } \\
\text { de discusión } \\
\text { política }\end{array}$ \\
\hline \multicolumn{6}{|l|}{ PP } \\
\hline Bastante & 3,9 & 6,9 & 2,4 & 0 & 0,7 \\
\hline Alguna & 28,3 & 23,3 & 20 & 1,7 & 4 \\
\hline \multicolumn{6}{|l|}{ vez } \\
\hline Nunca & 67,8 & 69,8 & 77,6 & 98,3 & 95,4 \\
\hline \multicolumn{6}{|l|}{ PSOE } \\
\hline Bastante & 9,4 & 15 & 5,8 & 0,6 & 1,5 \\
\hline Alguna & 37,1 & 25,9 & 35,2 & 3,6 & 4,7 \\
\hline \multicolumn{6}{|l|}{ vez } \\
\hline Nunca & 53,4 & 69,1 & 59 & 95,8 & 93,8 \\
\hline \multicolumn{6}{|l|}{ Podemos } \\
\hline Bastante & 23,8 & 18,9 & 18,1 & 1,1 & 9,2 \\
\hline Alguna & 46,6 & 39,1 & 45,5 & 12,5 & 15 \\
\hline \multicolumn{6}{|l|}{ vez } \\
\hline Nunca & 29,5 & 42 & 36,4 & 86,4 & 75,8 \\
\hline \multicolumn{6}{|l|}{ C's } \\
\hline Bastante & 8,3 & 12,7 & 4,7 & 0,1 & 2,4 \\
\hline Alguna & 44 & 34,1 & 39,4 & 3,4 & 7,3 \\
\hline \multicolumn{6}{|l|}{ vez } \\
\hline Nunca & 47,4 & 53,2 & 55,8 & 96,4 & 90,3 \\
\hline
\end{tabular}

Fuente: Elaboración propia a partir de los datos del CIS. Estudio nº3126 
La última variable del perfil sociodemográfico es la participación política en diferentes actividades más allá de la participación electoral. La Tabla 2 muestra la frecuencia con la que los votantes de los diferentes partidos se implican en actividades como asistir a una huelga autorizada, hacer boicot a productos por diversas razones (económicas, políticas, ecológicas...), participar en una huelga, ocupar edificios, participar en encierros o bloquear el tráfico y, por último, participar en sitios de discusión política por internet. Los datos de la tabla arrojan que las actividades en las que más participan los votantes (de todos los partidos) son la asistencia a manifestaciones y la participación en huelgas, mientras que las actividades en las que menos se implican son la ocupación, los encierros y el bloqueo del tráfico y la discusión política a través de internet. Amén de dichos datos, los votantes que más participan en cualquiera de estas actividades son los de Podemos, seguidos por el PSOE en lo que a manifestaciones y huelgas se refiere y de C's en actividades como el boicot a productos y el debate político en internet. Por su parte, el PP es el partido con menores cotas de participación entre sus votantes.

\section{Perfil Socioeconómico}

El último de los perfiles a analizar es el socioeconómico. La esfera económica siempre ha estado muy relacionada con la esfera política. En general, se suele pensar que las personas con mayores ingresos y puestos de trabajo más profesionales o mejor valorados suelen acercarse más a la derecha política, mientras que las personas con menores ingresos y puestos de trabajo menos cualificados se decantan por la izquierda. El primer análisis de este apartado da cuenta de la relación que existe entre los ingresos mensuales de los votantes y su propensión a votar a cada una de las cuatro fuerzas políticas.

La Figura 6 refleja, precisamente, cómo el nivel de ingresos de los electores influye en su tendencia de voto. Entre los partidos tradicionales, el $\mathrm{PP}$ es un partido que recoge, en su mayoría, votantes con un nivel mensual de ingresos de entre $600 €$ y $1200 €$ y el PSOE recoge una mayoría de ciudadanos cuyos ingresos se encuentran en el umbral de 600-900€. Podemos y C's, por otro lado, poseen un perfil de voto con un alto nivel de 


\section{Valero-Oteo - El Perfil del Votante del Cambio en España}

ingresos. C's reparte sus votos, sobre todo, entre personas con ingresos entre $1200 €$ y $1800 € \mathrm{y}$, en menor medida, de personas con ingresos superiores a $1800 €$. Podemos, por su parte, presenta una mayoría de votantes con un nivel de ingresos superior a los $1800 €$, aunque cabe destacar, que a este perfil de ingresos le sigue el de votantes con ingresos menores a $600 €$, por lo que tiene un perfil bastante polarizado. Esta variable se relaciona directamente con la de estatus socioeconómico. De esta manera, si el perfil socioeconómico mayoritario, por ejemplo, de Podemos es de personas de clase alta/media alta, es lógico que el nivel de ingresos predominante sea alto, al igual que si en el PSOE predominan los votantes de clase obrera, su perfil de ingresos mayoritario será menor.

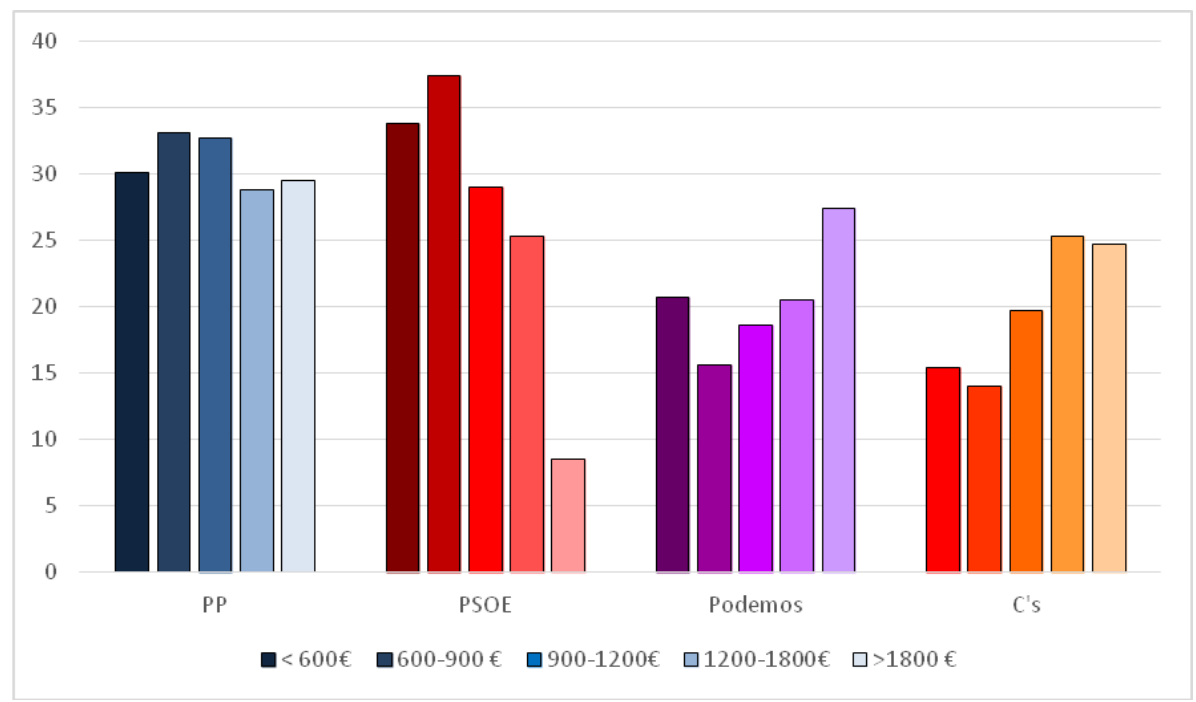

Figura 6: Recuerdo de voto (2015)*Ingresos

Fuente: Elaboración propia a partir de los datos del CIS. Estudio no 3124

En el caso de las elecciones europeas (Tabla 5, anexos), en 2014 el voto socialista estaba más precarizado; PP y C's mantenían un perfil similar al actual y Podemos presentaba un perfil de votante con menores ingresos (1200-1800€). Con esto se puede decir que la única variación importante, 
entre los nuevos partidos, ha sido el aumento en el nivel de ingresos del perfil del electorado de Podemos.

El segundo factor socioeconómico que se ha seleccionado en este trabajo es la valoración sobre la situación económica personal de los encuestados. Los partidos que albergan los votantes más conformes con su situación económica personal son el PP y C's, mientras que en las filas del PSOE y Podemos los votantes se caracterizan por calificar su situación como mala o muy mala. Con respecto a 2014 y a las elecciones europeas (Tabla 6, anexos) lo más destacable es que en 2014 el 50\% de las personas que se definían con una buena situación económica eran votantes de Podemos y, a su vez, éstas conformaban el grueso mayoritario de votantes del partido. C's, por su parte, se ha mantenido similar, con votantes con una buena situación económica personal.

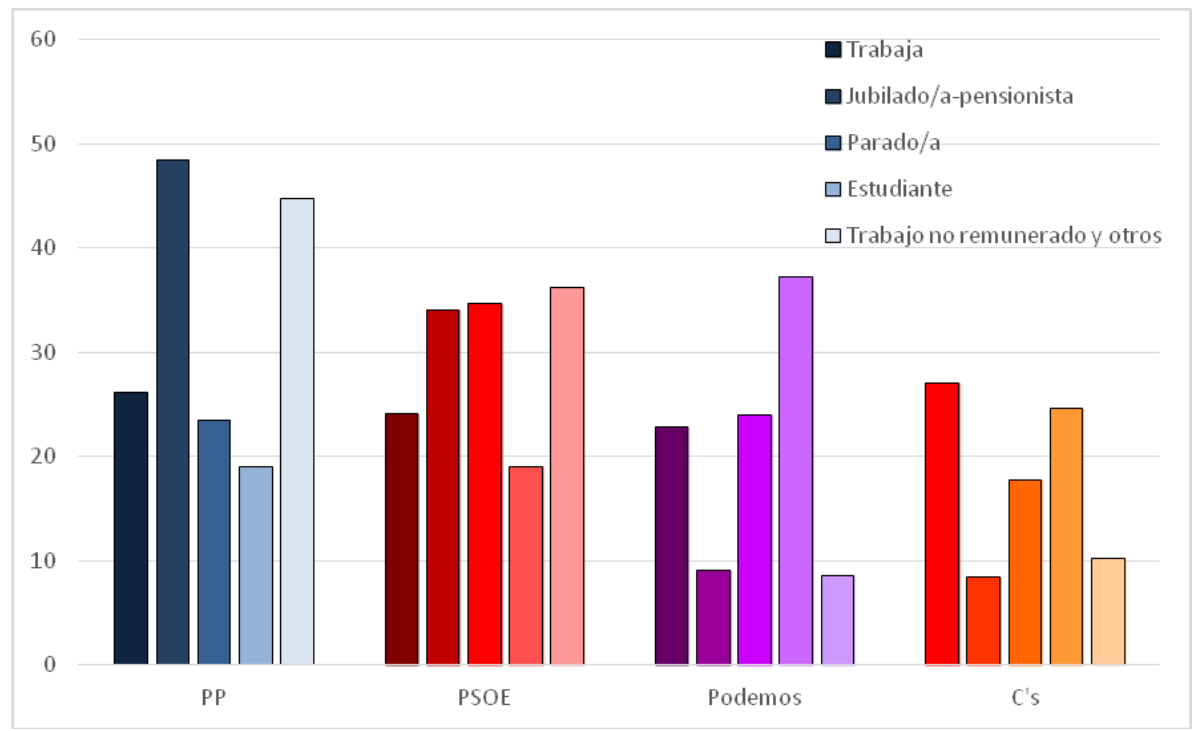

Figura 7: Recuerdo de voto (2015)*Situación laboral

Fuente: Elaboración propia a partir de los datos del CIS. Estudio n 3126.

La última de las variables analizadas es la situación laboral. En este caso lo que se busca es comprobar si existe una relación directa, entre la situación laboral y el voto, que permita conformar un tipo de perfil específico para 


\section{Valero-Oteo - El Perfil del Votante del Cambio en España}

cada partido. La Figura 7 lo corrobora. El PP en la actualidad es un partido formado sobre todo por pensionistas y jubilados, al igual que el PSOE, aunque este último incorpora también al grupo de los parados/as. Podemos es un partido compuesto en su mayoría por estudiantes (el 37,3\% de los encuestados estudiantes, manifestó haber votado a este partido en 2015) seguido por el grupo de los/as parados/as. Por último, C's posee un perfil mayoritario de trabajadores. Esta división de perfiles está muy relacionada con la variable edad. Si PP y PSOE son partidos muy envejecidos, lógicamente en sus filas tendrán un perfil mayoritario de jubilado/a, o de personas que se han quedado en paro a una edad adulta avanzada (como puede ser el caso del PSOE), mientras que los partidos más jóvenes estarán compuestos por personas en edad de trabajar y por estudiantes. Si se comparan estos resultados con los resultados de la encuesta postelectoral de las elecciones europeas, se observa cómo los perfiles de voto de los nuevos partidos apenas han variado.

\section{Últimas Consideraciones}

Se han elaborado ya diversos análisis relacionados con los resultados de las elecciones del 20D, como es el caso del CIS, o de las características de los votantes de los distintos partidos, como lo hizo Javier Elzo ${ }^{7}$ antes de las elecciones del 26J, en este trabajo se ha pretendido, además de eso, comparar los resultados del voto a las cuatro formaciones mayoritarias (haciendo hincapié en las neófitas) desde las elecciones al Parlamento Europeo. El principal problema de esta comparación es la diferencia entre la "importancia" unas elecciones europeas ("segundo orden") y unas generales ("primer orden") para la ciudadanía, pues en las europeas se considera que hay menos aspectos en juego que en los comicios de carácter nacional (Reif y Schmitt, 1980). Por otro lado, existe una gran diferencia de participación electoral entre unos comicios y otros, pues cuanto más complejo es el entramado político de un sistema electoral, más coste le supone al votante acudir a las urnas (Gallego, 2011). Finalmente, en unas elecciones europeas la intención con la que se emite el voto es distinta a la de unas elecciones generales. En este sentido, en unos comicios de segundo orden, el carácter del voto es más expresivo que instrumental, pues al existir menos aspectos 
en juego los electores pueden manifestar su voto de manera diferente, desde votar a partidos con mayor cercanía ideológica a la suya, quienes no gozarían de tantas posibilidades en una competición nacional (Belluci et al, 2012); hasta utilizar el voto europeo como voto de castigo hacia el gobierno nacional vigente en el momento de los comicios, como pudo ocurrir en las Elecciones al Parlamento Europeo de 2014. Por todo ello, es más complejo realizar una comparación entre los resultados electorales de comicios de distinto orden, sin embargo, para comparar los inicios de los partidos era necesario utilizar los resultados europeos. Quizá podrían haberse comparado los resultados de las elecciones del 20D y el 26J, sin embargo, los datos postelectorales de las elecciones de junio aún no han sido publicados $y$ después de los errores de medición de la encuesta preelectoral, no hubiera resultado un análisis "real" del voto a los partidos.

Dejando a un lado lo anterior, este trabajo es interesante para analizar cómo eran los votantes de las distintas fuerzas en 2014 y cómo han ido transformándose hasta el año 2015, pues no hay que olvidar que Podemos nació escasos meses antes de los comicios europeos y C's era un partido, hasta entonces, desconocido en un nivel nacional y fue después de dichas elecciones cuando comenzaron a hacerse visibles y conocidos por toda la ciudadanía.

\section{Conclusiones}

Con todos los análisis anteriores se confirma que existen características que definen a los nuevos partidos en contraposición a los partidos tradicionales. Las características que marcan esa barrera entre el voto de nuevos y viejos partidos son, sobre todo, de carácter sociodemográfico. Los primeros tienen más presencia entre los jóvenes urbanitas, con altos niveles de estudios y perteneciente a las nuevas clases medias o la clase media y media/alta; además de estar más presentes entre los votantes más interesados en política, críticos con la situación política del país y con mayor participación política más allá de las urnas, sobre todo en el caso de Podemos que es el partido con los votantes más participativos. Los partidos tradicionales, por el contrario, tienen una influencia más acusada entre los votantes del mundo rural, de edades más avanzadas (mayores de 50 años), con un nivel de estudios 


\section{Valero-Oteo - El Perfil del Votante del Cambio en España}

bastante inferior y con mucho menos interés político. En cuanto al factor clase social, PP y PSOE se distancian, el PP tiene más peso, sobre todo, entre las viejas clases medias y el PSOE entre la clase obrera; lo mismo ocurre con la valoración política del país, los que mejor la valoran tienden a votar al PP, mientras que el PSOE tiene un perfil de votante más crítico con la situación política.

En relación a la ideología y los factores socioeconómicos los perfiles de Podemos y C's dejan de converger. Quizá sea el perfil ideológico el que más distancia a los votantes de estos dos partidos. Podemos se ha convertido en un partido cuyo votante más representativo es de extrema izquierda e izquierda, mientras que C's es un partido compuesto por votantes del centro y la derecha del espectro político. Es decir, en términos ideológicos Podemos está más cerca del PSOE y C's del PP, tanto es así que se puede hablar de nueva izquierda y nueva derecha en España, nuevas ideologías que han absorbido a un importante número de votantes de los partidos tradicionales. Lo mismo ocurre a la hora de valorar la situación económica personal, los votantes de Podemos valoran su situación de manera muy negativa mientras que en C's ocurre lo opuesto. Bien es verdad que, respecto al nivel de ingresos, los dos partidos presentan un perfil de votante que supera los $1200 €$, aunque Podemos posee un perfil mayoritario con ingresos mensuales superiores al perfil de C's. Por último, Podemos tiene un perfil mayoritario de estudiantes y C's de personas que trabajan. Es este sentido, C's representaría a la población joven pero, sobre todo, de mediana edad, con alto nivel de estudios y una situación laboral y económica buena o muy buena, mientras que Podemos representaría a los jóvenes con alto nivel de estudios y con un poder adquisitivo bastante alto. Por lo tanto, sí que existen diferencias entre el perfil del voto del cambio y el perfil de voto tradicional, cuyo elemento de divergencia es la ideología.

Para terminar, cabe destacar que, en relación a las elecciones al Parlamento Europeo, los perfiles de voto de los dos nuevos partidos apenas han sufrido cambios. En lo que a edad se refiere dentro de Podemos se ha producido un aumento en la edad media de su votantes (de un año), al contrario que en C's, en el que se ha producido una disminución de la misma (de cuatro años) desde 2014 hasta 2015. Otro cambio que puede destacarse se relaciona con la autoubicación ideológica de los votantes. En el caso de 
Podemos, sus votantes han radicalizado su ideología desde 2014, aunque de forma muy leve, por otro lado, los de C's se han acercado más a la derecha ideológica, cambio más acusado que en el caso de Podemos. A pesar de ello las diferencias, como ya se ha dicho, son muy leves entre estos dos años para ambos partidos, es decir, sus perfiles de voto apenas han variado desde el debut de las formaciones en las elecciones europeas.

\section{Notas}

${ }^{1}$ Datos del CIS. Barómetro de abril 2015. Estudio n 3080

${ }^{2}$ Datos del CIS. Barómetro de abril 2015. Estudio n 3080

${ }^{3}$ Datos del Ministerio del Interior, http://resultadosgenerales2015.interior.es/congreso/\#/ES201512-CON-ES/ES

${ }^{4}$ En este trabajo se ha utilizado únicamente el porcentaje de columna, debido a la "regla de Zeisel" que se aplica "siempre que uno de los dos factores del cuadro dimensional pueda considerarse como causa de la distribución del otro factor. La regla es que los porcentajes deben computarse en el sentido del factor causal (Zeisel, 1962, 37).

${ }^{5}$ El CIS clasifica la categoría de estatus socioeconómico en los siguientes valores: clase alta/media alta (profesionales y técnicos/as, directivos/as y cuadros medios), nuevas clases medias (asalariados no manuales), viejas clases medias (empresarios/as, autónomos/as y agricultores/as), obreros/as cualificados/as (manuales cualificados/as, semicualificados/as, capataces y artesanos/as) y obreros/as no cualificados/as (obreros/as de la industria y los servicios y jornaleros/as del campo).

${ }^{6}$ La media ideológica se calcula en base a un eje ideológico, dispuesto en una escala del 1 al 10, donde 1 significa extrema izquierda y 10 , extrema derecha.

7 "Perfil sociológico de los votantes a las cinco formaciones mayores el 26J", disponible en http://javierelzo.blogspot.com.es/2016/05/perfil-sociologico-de-los-votantes-las.html, consultado 04/09/2016.

\section{Referencias}

Cabrera, P. (2014, 30 de junio). ¿Qué supone la irrupción de Podemos en la izquierda? Politikon. Recuperado de http://politikon.es/

Bellucci, P., Garzia, D., y Rubal, M. (2012). ¿Importa Europa en las elecciones europeas? Un modelo explicativo de las elecciones del 2009 al Parlamento Europeo. Revista Española de Investigaciones Sociológicas, 137, 25-41. doi:10.5477/cis/reis.137.25 
Escobar, M., Fernández, E., y Bernardi, F. (2010). Análisis de datos con Stata. Madrid: Centro de Investigaciones Sociológicas.

Estefanía, J. et al. (Dir.) (2015). Informe sobre la democracia 2015. Reformular la política. Madrid: Fundación Alternativas.

Galindo, J., Llaneras, K., Medina, O., San Miguel, J., Simón, P. y Senserrich, R. (2015). La urna rota. La crisis política e institucional del modelo español. Barcelona: Debate.

Gallego, A. (2011). Are more choices in the ballot better? Cross-Nacional and experimental evidence. APSA Annual Meeting Papers.

González, J., y Caínzos, M. (2012). Ciclos políticos y comportamiento electoral de jóvenes y mayores en España, 1979-2011. Panorama Social, 15, 165-179.

Hirschmann, A.O. (1977). Salida, Voz y Lealtad: respuestas al deterioro de empresas, organizaciones y estados. México: Fondo de Cultura Económica.

Lago, I. (1999). Sobrerrepresentación de las zonas rurales y voto conservador en el sistema electoral español. Dereito, 1(8), 81-101.

Laparra, M., y Pérez Eransus, B. (2012). Crisis y fractura social en Europa: causas y efectos en España. Barcelona: Obra Social La Caixa.

Noelle-Neumann, E. (2010). La espiral del silencio: opinión pública: nuestra piel social. Barcelona: Paidós.

Reif, K., y Schmitt, H. (1980). Nine second-order national elections: A conceptual framework for the analysis of European election results. European Journal of Political Research, 8(1), 3-44. doi: 10.1111/j.1475-6765.1980.tb00737.x

Salido, O., y Martín, A. (2007). Las urnas de la precariedad. El anclaje sociolaboral del voto juvenil en el 14-M. Sociedad y Utopía: Revista de Ciencias Sociales, 29, 463-488.

Sánchez-Cuenca, I. (2014). La impotencia democrática. Madrid: Catarata.

Zeisel, H. (1962). Dígalo con números. Madrid: Fondo de Cultura Económica. 


\section{Anexos}

Tabla 1

Recuerdo de voto*hábitat

\begin{tabular}{|c|c|c|c|c|c|c|}
\hline & & & \multicolumn{3}{|c|}{ Hábitat } & \multirow[b]{3}{*}{ Total } \\
\hline & & & $</=$ & Entre 10.000 & $>$ & \\
\hline & & & 10.000 & y 100.000 & 100.000 & \\
\hline \multirow{12}{*}{$\begin{array}{l}\text { Recuerdo de voto } \\
\text { (2015) }\end{array}$} & \multirow{3}{*}{ PP } & Recuento & 333 & 425 & 113 & 1203 \\
\hline & & $\%$ dentro de Hábitat & $38,6 \%$ & $31,6 \%$ & $34,8 \%$ & $33,2 \%$ \\
\hline & & Residuos corregidos & 3,9 & $-1,6$ & $-1,8$ & \\
\hline & \multirow{3}{*}{ PSOE } & Recuento & 310 & 426 & 336 & 1072 \\
\hline & & \% dentro de Hábitat & $35,9 \%$ & $31,6 \%$ & $23,7 \%$ & $29,6 \%$ \\
\hline & & Residuos corregidos & 4,7 & 2,1 & $-6,2$ & \\
\hline & \multirow{3}{*}{ Podemos } & Recuento & 113 & 221 & 339 & 673 \\
\hline & & \% dentro de Hábitat & $13,1 \%$ & $16,4 \%$ & $23,9 \%$ & $18,6 \%$ \\
\hline & & Residuos corregidos & $-4,7$ & $-2,5$ & 6,7 & \\
\hline & \multirow{3}{*}{ C's } & Recuento & 107 & 274 & 297 & 678 \\
\hline & & \% dentro de Hábitat & $12,4 \%$ & $20,4 \%$ & $21,0 \%$ & $18,2 \%$ \\
\hline & & Residuos corregidos & $-5,4$ & 2 & 2,8 & \\
\hline \multirow{2}{*}{ Total } & & Recuento & 863 & 1346 & 1417 & 3626 \\
\hline & & $\%$ dentro de Hábitat & $100,0 \%$ & $100,0 \%$ & $100,0 \%$ & $100,0 \%$ \\
\hline
\end{tabular}

Fuente: Elaboración propia a partir de los datos del CIS 


\section{Valero-Oteo - El Perfil del Votante del Cambio en España}

Tabla 2

Recuerdo de voto (2015)*Nivel de estudios

\begin{tabular}{|c|c|c|c|c|c|c|}
\hline & & & $\begin{array}{c}\text { Estudios } \\
\text { Primarios }\end{array}$ & $\begin{array}{c}\text { Estudios } \\
\text { secundarios }\end{array}$ & $\begin{array}{c}\text { Estudios } \\
\text { superiores }\end{array}$ & Total \\
\hline \multirow{12}{*}{$\begin{array}{l}\text { Recuerdo } \\
\text { de voto } \\
\text { (2015) }\end{array}$} & \multirow{3}{*}{ PP } & Recuento & 403 & 566 & 228 & 1197 \\
\hline & & $\begin{array}{l}\text { \%dentro de } \\
\text { Estudios }\end{array}$ & $43,1 \%$ & $29,9 \%$ & $29,5 \%$ & \multirow[t]{2}{*}{$33,2 \%$} \\
\hline & & $\begin{array}{l}\text { Residuos } \\
\text { tipificados }\end{array}$ & 7,4 & $-4,5$ & $-2,5$ & \\
\hline & \multirow{3}{*}{ PSOE } & Recuento & 292 & 551 & 124 & 1067 \\
\hline & & $\begin{array}{l}\text { \% dentro de } \\
\text { Estudios }\end{array}$ & $40,9 \%$ & $29,1 \%$ & $16,0 \%$ & \multirow[t]{2}{*}{$29,6 \%$} \\
\hline & & $\begin{array}{l}\text { Residuos } \\
\text { tipificados }\end{array}$ & 9,6 &,- 7 & $-9,3$ & \\
\hline & \multirow{3}{*}{ Podemos } & Recuento & 76 & 393 & 199 & 668 \\
\hline & & $\begin{array}{l}\text { \% dentro de } \\
\text { Estudios }\end{array}$ & $8,1 \%$ & $20,7 \%$ & $25,7 \%$ & \multirow[t]{2}{*}{$18,5 \%$} \\
\hline & & $\begin{array}{l}\text { Residuos } \\
\text { tipificados }\end{array}$ & $-9,5$ & 3,6 & 5,8 & \\
\hline & \multirow{3}{*}{$\mathrm{Cs}_{\mathrm{s}}$} & Recuento & 65 & 384 & 222 & 671 \\
\hline & & $\begin{array}{l}\% \text { dentro de } \\
\text { Estudios }\end{array}$ & $6,9 \%$ & $20,3 \%$ & $28,7 \%$ & \multirow[t]{2}{*}{$18,6 \%$} \\
\hline & & $\begin{array}{l}\text { Residuos } \\
\text { tipificados }\end{array}$ & $-10,7$ & 2,7 & 8,1 & \\
\hline \multirow[b]{2}{*}{ Total } & & Recuento & 677 & 1894 & 773 & 3602 \\
\hline & & $\begin{array}{l}\% \text { dentro de } \\
\text { Estudios }\end{array}$ & $100,0 \%$ & $100,0 \%$ & $100,0 \%$ & $100,0 \%$ \\
\hline
\end{tabular}

Fuente: Elaboración propia a partir de los datos del CIS. Estudio n ${ }^{\circ} 3126$ 
Tabla 3

Recuerdo de voto (2015)*Autoubicación Ideológica

\begin{tabular}{|c|c|c|c|c|c|c|c|c|}
\hline & & & \multicolumn{5}{|c|}{ Ideología } & \multirow[b]{2}{*}{ Total } \\
\hline & & & $\begin{array}{c}\text { Extrema } \\
\text { izda. }\end{array}$ & Izquierda & Centro & Derecha & $\begin{array}{c}\text { Extrema } \\
\text { dcha }\end{array}$ & \\
\hline \multirow{12}{*}{$\begin{array}{l}\text { Recuerdo } \\
\text { de voto } \\
\text { (2015) }\end{array}$} & \multirow{3}{*}{ PP } & Recuento & 2 & 18 & 409 & 512 & 150 & 1091 \\
\hline & & $\begin{array}{l}\text { \% dentro de } \\
\text { Ideología }\end{array}$ &, $6 \%$ & $1,6 \%$ & $36,2 \%$ & $84,6 \%$ & $90,4 \%$ & \multirow[t]{2}{*}{$32,7 \%$} \\
\hline & & $\begin{array}{l}\text { Residuos } \\
\text { tipificados }\end{array}$ & $-9,9$ & $-18,2$ & 2,0 & 22,3 & 13,0 & \\
\hline & \multirow{3}{*}{ PSOE } & Recuento & 124 & 665 & 189 & 11 & 1 & \multirow{3}{*}{$\begin{array}{c}990 \\
29,7 \%\end{array}$} \\
\hline & & $\begin{array}{l}\text { \% dentro de } \\
\text { Ideología }\end{array}$ & $40,0 \%$ & $59,2 \%$ & $16,7 \%$ & $1,8 \%$ &, $6 \%$ & \\
\hline & & $\begin{array}{l}\text { Residuos } \\
\text { tipificados }\end{array}$ & 3,3 & 18,1 & $-8,0$ & $-12,6$ & $-6,9$ & \\
\hline & \multirow{3}{*}{ Podemos } & Recuento & 180 & 341 & 105 & 2 & 0 & 628 \\
\hline & & $\begin{array}{l}\text { \% dentro de } \\
\text { Ideología }\end{array}$ & $58,1 \%$ & $30,3 \%$ & $9,3 \%$ &, $3 \%$ &, $0 \%$ & \multirow[t]{2}{*}{$18,8 \%$} \\
\hline & & $\begin{array}{l}\text { Residuos } \\
\text { tipificados }\end{array}$ & 15,9 & 8,9 & $-7,4$ & $-10,5$ & $-5,6$ & \\
\hline & \multirow{3}{*}{$C_{s}^{\prime}$} & Recuento & 4 & 100 & 428 & 80 & 15 & 627 \\
\hline & & $\begin{array}{l}\text { \% dentro de } \\
\text { Ideología }\end{array}$ & $1,3 \%$ & $8,9 \%$ & $37,8 \%$ & $13,2 \%$ & $9,0 \%$ & \multirow[t]{2}{*}{$18,8 \%$} \\
\hline & & $\begin{array}{l}\text { Residuos } \\
\text { tipificados }\end{array}$ & $-7,1$ & $-7,7$ & 14,8 & $-3,2$ & $-2,9$ & \\
\hline \multirow[b]{2}{*}{ Total } & & Recuento & 310 & 1124 & 1131 & 605 & 166 & 3336 \\
\hline & & $\begin{array}{l}\text { \% dentro de } \\
\text { Ideología }\end{array}$ & $100,0 \%$ & $100,0 \%$ & $100,0 \%$ & $100,0 \%$ & $100,0 \%$ & $100,0 \%$ \\
\hline
\end{tabular}

Fuente: Elaboración propia a partir de los datos del CIS. Estudio no 3126 


\section{Valero-Oteo - El Perfil del Votante del Cambio en España}

Tabla 4

Recuerdo de voto (2015)*Grado de interés en la campaña electoral Elecciones Generales 2015

\begin{tabular}{|c|c|c|c|c|c|c|c|}
\hline & & & \multicolumn{4}{|c|}{ Interés campaña electoral elecciones generales de 2015} & \multirow[b]{2}{*}{ Total } \\
\hline & & & $\begin{array}{c}\text { Con } \\
\text { mucho } \\
\text { interés }\end{array}$ & $\begin{array}{c}\text { Con } \\
\text { bastante } \\
\text { interés }\end{array}$ & $\begin{array}{l}\text { Con poco } \\
\text { interés }\end{array}$ & $\begin{array}{l}\text { Con } \\
\text { ningún } \\
\text { interés }\end{array}$ & \\
\hline \multirow{12}{*}{$\begin{array}{l}\text { Recuerdo } \\
\text { de voto } \\
(2015)\end{array}$} & \multirow{3}{*}{ PP } & Recuento & 202 & 469 & 376 & 151 & 1198 \\
\hline & & $\begin{array}{l}\text { \% dentro de Interés } \\
\text { campaña }\end{array}$ & $29,0 \%$ & $34,0 \%$ & $33,2 \%$ & $36,7 \%$ & \multirow[t]{2}{*}{$33,1 \%$} \\
\hline & & Residuos corregidos & $-2,6$ &, 9 &, 1 & 1,7 & \\
\hline & \multirow{3}{*}{ PSOE } & Recuento & 151 & 361 & 399 & 158 & 1069 \\
\hline & & $\begin{array}{l}\% \text { dentro Interés } \\
\text { campaña }\end{array}$ & $21,7 \%$ & $26,2 \%$ & $35,3 \%$ & $38,4 \%$ & \multirow[t]{2}{*}{$29,6 \%$} \\
\hline & & Residuos corregidos & $-5,1$ & $-3,5$ & 5,1 & 4,2 & \\
\hline & \multirow{3}{*}{ Podemos } & Recuento & 201 & 270 & 149 & 52 & 672 \\
\hline & & $\begin{array}{l}\text { \% dentro de Interés } \\
\text { campaña }\end{array}$ & $28,8 \%$ & $19,6 \%$ & $13,2 \%$ & $12,7 \%$ & \multirow[t]{2}{*}{$18,6 \%$} \\
\hline & & Residuos corregidos & 7,8 & 1,2 & $-5,6$ & $-3,3$ & \\
\hline & \multirow{3}{*}{$C^{\prime} s$} & Recuento & 143 & 278 & 207 & 50 & 678 \\
\hline & & $\begin{array}{l}\text { \% dentro de Interés } \\
\text { campaña }\end{array}$ & $20,5 \%$ & $20,2 \%$ & $18,3 \%$ & $12,2 \%$ & \multirow[t]{2}{*}{$18,7 \%$} \\
\hline & & Residuos corregidos & 1,3 & 1,7 & -5 & $-3,6$ & \\
\hline \multirow[b]{2}{*}{ Total } & & Recuento & 697 & 1378 & 1131 & 411 & 3617 \\
\hline & & $\begin{array}{l}\text { \% dentro de Interés } \\
\text { campaña }\end{array}$ & $100,0 \%$ & $100,0 \%$ & $100,0 \%$ & $100,0 \%$ & $\begin{array}{c}100,0 \\
\% \\
\end{array}$ \\
\hline
\end{tabular}

Fuente: Elaboración propia a partir de los datos del CIS. Estudio no 3126 
Tabla 5

Recuerdo de voto (2014)*Nivel de ingresos

\begin{tabular}{|c|c|c|c|c|c|c|c|c|}
\hline & & & \multicolumn{5}{|c|}{ Ingresos } & \multirow[b]{2}{*}{ Total } \\
\hline & & & $<600 €$ & $\begin{array}{l}600- \\
900 €\end{array}$ & $\begin{array}{c}900- \\
1200 €\end{array}$ & $\begin{array}{l}1200- \\
1800\end{array}$ & $\begin{array}{c}>1800 \\
€\end{array}$ & \\
\hline \multirow{12}{*}{$\begin{array}{l}\text { Recuerdo } \\
\text { de voto } \\
(2014)\end{array}$} & \multirow{3}{*}{ PP } & Recuento & 78 & 44 & 40 & 32 & 20 & 214 \\
\hline & & $\%$ dentro de Ingresos & $28,2 \%$ & $36,1 \%$ & $35,7 \%$ & $39,5 \%$ & $42,6 \%$ & $33,5 \%$ \\
\hline & & Residuos corregidos & $-2,5$ &, 7 &, 5 & 1,2 & 1,4 & \\
\hline & \multirow{3}{*}{ PSOE } & Recuento & 121 & 47 & 40 & 18 & 18 & 244 \\
\hline & & $\%$ dentro de Ingresos & $43,7 \%$ & $38,5 \%$ & $35,7 \%$ & $22,2 \%$ & $38,3 \%$ & $38,2 \%$ \\
\hline & & Residuos corregidos & 2,5 &, 1 &,- 6 & $-3,2$ & 0 & \\
\hline & \multirow{3}{*}{ Podemos } & Recuento & 71 & 24 & 29 & 23 & 4 & 151 \\
\hline & & $\%$ dentro de Ingresos & $25,6 \%$ & $19,7 \%$ & $25,9 \%$ & $28,4 \%$ & $8,5 \%$ & $23,6 \%$ \\
\hline & & Residuos corregidos & 1,0 & $-1,1$ & 6 & 1,1 & $-2,5$ & \\
\hline & \multirow{3}{*}{$C^{\prime} s$} & Recuento & 7 & 7 & 3 & 8 & 5 & 30 \\
\hline & & $\%$ dentro de Ingresos & $2,5 \%$ & $5,7 \%$ & $2,7 \%$ & $9,9 \%$ & $10,6 \%$ & $4,7 \%$ \\
\hline & & Residuos corregidos & $-2,3$ &, 6 & $-1,1$ & 2,4 & 2,0 & \\
\hline \multirow[b]{2}{*}{ Total } & & Recuento & 277 & 122 & 112 & 81 & 47 & 639 \\
\hline & & $\%$ dentro de Ingresos & $100,0 \%$ & $100,0 \%$ & $100,0 \%$ & $100,0 \%$ & $\begin{array}{c}100,0 \\
\%\end{array}$ & $\begin{array}{c}100,0 \\
\% \\
\end{array}$ \\
\hline
\end{tabular}

Fuente: Elaboración propia a partir de los datos del CIS. Estudio n ${ }^{\circ} 3028$ 


\section{Valero-Oteo - El Perfil del Votante del Cambio en España}

Tabla 6

Recuerdo de voto (2014)*Valoración de la situación económica personal

\begin{tabular}{|c|c|c|c|c|c|c|c|c|}
\hline & & & \multicolumn{5}{|c|}{ Valoración de la situación económica personal } & \multirow[b]{2}{*}{ Total } \\
\hline & & & $\begin{array}{c}\text { Muy } \\
\text { buena }\end{array}$ & Buena & Regular & Mala & $\begin{array}{l}\text { Muy } \\
\text { mala }\end{array}$ & \\
\hline \multirow{12}{*}{$\begin{array}{l}\text { Recuerdo } \\
\text { de voto } \\
\text { (2014) }\end{array}$} & \multirow{3}{*}{ PP } & Recuento & 4 & 115 & 131 & 29 & 7 & 286 \\
\hline & & $\begin{array}{l}\text { \% dentro situación } \\
\text { económica personal }\end{array}$ & $50,0 \%$ & $45,6 \%$ & $34,3 \%$ & $24,6 \%$ & $16,3 \%$ & \multirow[t]{2}{*}{$35,6 \%$} \\
\hline & & Residuos corregidos & 9 & 4,0 &,- 7 & $-2,7$ & $-2,7$ & \\
\hline & \multirow{3}{*}{ PSOE } & Recuento & 0 & 70 & 144 & 51 & 20 & 285 \\
\hline & & $\begin{array}{l}\text { \% dentro de situación } \\
\text { económica personal }\end{array}$ &, $0 \%$ & $27,8 \%$ & $37,7 \%$ & $43,2 \%$ & $46,5 \%$ & \multirow[t]{2}{*}{$35,5 \%$} \\
\hline & & Residuos corregidos & $-2,1$ & $-3,1$ & 1,2 & 1,9 & 1,6 & \\
\hline & \multirow{3}{*}{ Podemos } & Recuento & 4 & 55 & 91 & 33 & 14 & 197 \\
\hline & & $\begin{array}{l}\text { \% dentro de situación } \\
\text { económica personal }\end{array}$ & $50,0 \%$ & $21,8 \%$ & $23,8 \%$ & $28,0 \%$ & $32,6 \%$ & \multirow[t]{2}{*}{$24,5 \%$} \\
\hline & & Residuos corregidos & 1,7 & $-1,2$ &,- 4 &, 9 & 1,3 & \\
\hline & \multirow{3}{*}{$\mathrm{C}$ 's } & Recuento & 0 & 12 & 16 & 5 & 2 & 35 \\
\hline & & $\begin{array}{l}\text { \% dentro de situación } \\
\text { económica personal }\end{array}$ &, $0 \%$ & $4,8 \%$ & $4,2 \%$ & $4,2 \%$ & $4,7 \%$ & \multirow[t]{2}{*}{$4,4 \%$} \\
\hline & & Residuos corregidos &,- 6 &, 4 &,- 2 &,- 1 &, 1 & \\
\hline \multirow[b]{2}{*}{ Total } & & Recuento & 8 & 252 & 382 & 118 & 43 & 803 \\
\hline & & $\begin{array}{l}\% \text { dentro de situación } \\
\text { económica personal }\end{array}$ & $100,0 \%$ & $100,0 \%$ & $100,0 \%$ & $100,0 \%$ & $100,0 \%$ & $100,0 \%$ \\
\hline
\end{tabular}

Fuente: Elaboración propia a partir de los datos del CIS. Estudio no 3028

Irene Valero-Oteo es estudiante del programa de Doctorado

Sociedad, Política y Cultura de la Universidad del País Vasco, España

Dirección de contacto: Departamento de Sociología II. Facultad de

Ciencias Sociales y de la Comunicación. Universidad del País Vasco.

Barrio Sarriena, s/n -48940- Leioa (Bizkaia), España. Email:

irenevaleroteo@gmail.com 


\section{RIMCIS \\ Social Sciences}

\section{Hipatia Press}

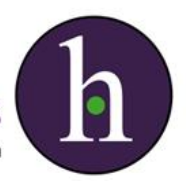

Instructions for authors, subscriptions and further details:

http://rimcis.hipatiapress.com

\section{Soluciones TIC para Personas Mayores: Preferencias Manifestadas en el Medio Rural Español}

María Ángeles Rubio Pastor ${ }^{1}$, Inmaculada Plaza García ${ }^{1}$, Víctor Orive Serrano ${ }^{1}$

1) Universidad de Zaragoza, España

Date of publication: July $30^{\text {th }}, 2017$

Edition period: July 2017 - November 2017

To cite this article: Rubio Pastor, M.A., Plaza García, I., \& Orive Serrano,

V. (2017). Soluciones TIC para Personas Mayores: Preferencias

Manifestadas en el Medio Rural Español. International and Multidisciplinary Journal of Social Sciences, 6(2), 137-177. doi: 10.17583/rimcis.2017.2221

To link this article: http://doi.org/10.17583/rimcis.2017.2221

\section{PLEASE SCROLL DOWN FOR ARTICLE}

The terms and conditions of use are related to the Open Journal System and to Creative Commons Attribution License (CC-BY). 


\section{ICT Solutions for Elder People: Manifested Preferences in Spanish Rural Areas}

María Ángeles Rubio Pastor

CUD-Universidad Zaragoza
Inmaculada Plaza García

Universidad de Zaragoza

Víctor Orive Serrano

Universidad de Zaragoza

\section{Abstract}

Aging and depopulation are serious problems in Spanish interior rural areas. As a result, it is difficult to provide older people with personalized care in these villages. At the same time, research and development of Information and Communication Technology (ICT) achieve relevance. It could play the role of family or neighbors to provide several care services. Thus, it is necessary to know the acceptance of older people about technological solutions. 122 elders participated in eleven workshops where they evaluated these solutions. Results reveal a good attitude towards devices and functionalities. This paper points out the preferences showed by older people about technological solutions. It could be a starting point for researchers to develop technology as a help for elderly in rural area.

Keywords: elderly, rural areas, technological device, ICT, technological applications 


\section{Soluciones TIC para Personas Mayores: Preferencias Manifestadas en el Medio Rural Español}

María Ángeles Rubio Pastor CUD- Universidad Zaragoza
Inmaculada Plaza García

Universidad de Zaragoza

Víctor Orive Serrano

Universidad de Zaragoza

\section{Resumen}

España sufre un problema de despoblación y envejecimiento en el medio rural del interior peninsular. Uno de sus efectos directos es la falta de personas que puedan ofrecer una atención cercana a aquellos mayores que aún permanecen en sus pueblos. Simultáneamente, se está observando un importante avance en la investigación y desarrollo de nuevos dispositivos y funcionalidades de las tecnologías de la información y la comunicación (TIC), que podrían suplir algunos cuidados que hasta ahora han venido realizando familiares o vecinos. Se considera preciso conocer el grado de aceptación de estas soluciones por parte de las personas mayores y para ello se ha reunido a ciento veintidós (122) ancianos en once (11) talleres, donde se les han mostrado mediante dinámicas de grupo y se les ha pedido que las valoraran. Los resultados han revelado una predisposición positiva de los potenciales usuarios, tanto hacia dispositivos como hacia funcionalidades, y han puesto de manifiesto sus preferencias ante este tipo de tecnologías. Estos resultados pueden servir a otros investigadores como referencia y punto de partida para el desarrollo de tecnología, en aras de mejorar la calidad de vida de los mayores en el medio rural.

Palabras clave: personas mayores, medio rural, dispositivos TIC, funcionalidades TIC

2017 Hipatia Press

ISSN: 2014-3680

DOI: $10.17583 /$ rimcis.2017.2221 


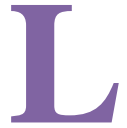

a evolución demográfica que en el medio rural del interior de España ha sufrido en las últimas décadas ha derivado en dos fenómenos preocupantes, despoblación y envejecimiento. Estos tienen un efecto directo sobre las condiciones sociales que se crean en el medio rural y sobre las que es preciso aportar nuevas soluciones, para posibilitar la permanencia de las personas mayores ${ }^{1}$ en sus hogares sin pérdida de calidad de vida.

Muchas de las necesidades de los mayores son atendidas ahora con mayor dificultad, ya que no queda en el medio rural quien pueda hacerlo: la familia, que tradicionalmente se ha hecho cargo de su cuidado, en muchos casos no vive ya en el pueblo, pues emigró al medio urbano en busca de trabajo; la familia que aún queda y concretamente las mujeres ven cada vez más difícil estar al cuidado de sus mayores, pues tienen otro tipo de responsabilidades; los vecinos, que siempre habían constituido una fuerte red social de atención mutua, van abandonando sus casas (García, 1997); y, aunque se hacen esfuerzos, los servicios públicos no logran sustituir a todos estos actores en la atención a los mayores.

Como efecto derivado, se observa que envejecer en un pequeño pueblo del interior de España conlleva diferencias significativas respecto a lo que sucede en el medio urbano. La diferencia más importante es que en el medio rural los mayores son el grupo de edad más numeroso, representando un elevado porcentaje de la población total. A esto se añade que el medio rural también cuenta con mayor tasa de masculinización. Y, en definitiva, esto se traduce en que el número de personas que necesitan cuidado es elevado, y pocas las personas jóvenes que puedan prestarlo, con lo que se incrementa la vulnerabilidad y la pérdida de autonomía de aquellos mayores que todavía tienen buena condición física y mental, y que podrían seguir disfrutando de su casa y su pueblo. En consecuencia, en el medio rural del interior de España se dan unas claves diferentes en el proceso de envejecimiento, que en muchas ocasiones llevan a que los mayores tengan que abandonar su pueblo ante la percepción de inseguridad, soledad y aislamiento (García, 1997; Triadó, 2003).

Así pues, esta realidad de las personas mayores del medio rural del interior de España obliga a implementar soluciones innovadoras que conduzcan a resultados distintos. Si la despoblación, el envejecimiento y la 
falta de servicios imposibilitan un cuidado humano y personalizado, resulta necesario pensar en otro tipo de soluciones tecnológicas que, en la medida de lo posible, puedan sustituir el trabajo de otro tipo de cuidadores.

En los últimos años se ha producido un considerable avance en la investigación y desarrollo de nuevas aplicaciones de las tecnologías de la información y la comunicación (TIC), que permite pensar en una solución factible cuando estas tecnologías estén diseñadas para responder a las necesidades de las personas mayores. Ya en otros campos se ha observado una rápida introducción de las TIC como solución innovadora. En varios estudios publicados en diferentes áreas de investigación (ergonomía, informática, psicología, economía) se han demostrado de manera convincente los beneficios que puede aportar la tecnología. Surge el concepto de "Internet de las cosas" (Internet of Things, IoT) (Atzori et al., 2010) por el que diferentes objetos cotidianos, dotados de pequeños sensores y conectados a través de redes, permiten gestionar las diferentes actividades diarias, sirviendo de apoyo en la vida cotidiana.

Hoy en día son muy importantes los avances que se están viviendo en el ámbito de las TIC, para el apoyo del día a día de las personas mayores. Esto puede contribuir a ampliar su autonomía, hacerles sentir más seguras y, en definitiva, posibilitar por más tiempo la permanencia en sus casas. Así, se acuñan nuevas áreas de investigación, tales como "Gerontecnología", centrada en la tecnología que busca responder a las necesidades de una sociedad en rápido proceso de envejecimiento. Más formalmente, Gerontecnología es definida como el estudio de la tecnología para garantizar un buen estado de salud, plena participación social y una autonomía garantizada a lo largo de la vida (Harrington \& Harrington, 2000). Algunas de las áreas específicas de investigación en Gerontecnología incluyen el lugar de trabajo y el hogar, la vida independiente atendiendo a la movilidad y la comunicación, el cuidado de la salud y la atención médica en el hogar, el ocio y la ayuda del cuidador (Comeau, 2005; Fernández-Caballero, 2017).

Sin embargo, no se puede obviar un prejuicio vinculado con las personas mayores en su uso de las TIC (por estar poco introducidas en la cultura de este tipo de tecnologías). En principio se presupone que muestran rechazo hacia el uso de estas tecnologías y miedo a enfrentarse a dispositivos desconocidos para ellos (Selwyn et al., 2003). Este rechazo puede estar 
provocado intrínsecamente, pero el entorno social también juega un importante papel a la hora de desanimarles o, al contrario, fomentar el uso de la tecnología (Barnard, 2013). Poco a poco estas consideraciones se van relajando, pues se observa que los mayores reconocen y valoran los beneficios que encierra el uso de la tecnología para facilitar su independencia (Mitzner et al., 2010). Aun con todo, es preciso reconocer que todavía existen ciertas reticencias que han sido objeto de estudio por numerosos autores. Estos estudios han puesto en evidencia que los factores que inciden en mayor medida en estas reticencias son, fundamentalmente: cuando el mayor percibe que la tecnología no logra satisfacer sus necesidades y cuando el mayor percibe cierta dificultad en el uso (Chau, 1996; Mitzner et al., 2010; Wang et al., 2011).

La aceptación de la tecnología se considera el primer paso para el éxito en el desarrollo de dispositivos técnicos y nuevas funcionalidades. Para reducir las reticencias de los mayores es preciso analizar cómo las TIC pueden contribuir a la satisfacción de sus verdaderas necesidades y cómo debe ser el diseño con el objeto de facilitar su uso. Así pues, aquí comienza el camino para la investigación, centrada en conocer las características especiales del colectivo de usuarios de mayor edad residente en el medio rural.

Apoyado en anteriores estudios (Rubio et al., 2013, Rubio et al., 2016), este análisis pretende mostrar cuáles son las prioridades de los habitantes mayores del medio rural en su elección de aquellos dispositivos y funcionalidades TIC que mejor puedan servir para responder a las necesidades que se les presentan en su día a día. Los resultados de este análisis pueden servir a futuros avances en la materia, aportando a los investigadores valiosa información sobre las preferencias manifestadas por sus potenciales usuarios.

\section{Objetivo: Las Soluciones Tecnológicas Deben Surgir de las Preferencias Manifestadas por sus Potenciales Usuarios}

Es obvio que el avance en las TIC lleva aparejadas continuas novedades y eso, a priori, no siempre asegura que puedan tener una aceptación positiva por parte de un colectivo como el de las personas mayores. En muchas ocasiones se idean soluciones técnicamente perfectas que, sin embargo, no 
consiguen responder a las preferencias de sus potenciales usuarios ya que no se les ha tenido suficientemente en cuenta.

La falta de satisfacción de necesidades explica la reticencia de las personas mayores al uso de dispositivos tecnológicos. No los consideran "útiles" pues no perciben ventajas o beneficios derivados de su uso (Wilkowska \& Ziefle, 2009). Por lo tanto, para que los mayores puedan conocer la ayuda ofrecida por las TIC es preciso hacer un ejercicio de pedagogía y mostrarles información extensa y de la forma más apropiada.

En consecuencia, es preciso ofrecer aquellos dispositivos y funcionalidades que efectivamente resulten atractivos a las personas que han de utilizarlos: "Aunque la oferta tecnológica a disposición del mayor es cada vez más variada, el teléfono móvil, Internet y la teleasistencia, son las que tienen mayor aceptación entre este grupo de población.” Pero es necesario también atender a las características y atributos relacionados con su potencial usabilidad: "Los mayores reclaman un diseño tecnológico pensado para ellos; quieren aparatos manejables, fáciles de aprender, con funciones muy básicas y de acceso sencillo" (Fernández, 2011). Así pues, el objetivo de este trabajo es conocer los dispositivos y funcionalidades TIC que mejor puedan responder a las necesidades de las personas mayores del medio rural, permitiéndoles acceder a un conjunto de servicios que pueden facilitar su vida autónoma en sus propios hogares.

Alcanzar este objetivo general exige abordar otros objetivos más concretos:

- Analizar y realizar una preselección de los dispositivos tecnológicos existentes en el mercado (teléfonos móviles, Tablet PC, Smartphone, ordenadores personales, TV, etc.), que puedan servir a las necesidades de las personas mayores.

- Analizar y realizar una preselección de las distintas funcionalidades que podrían dar respuesta a las necesidades más relevantes de los mayores del medio rural.

- Someter los dispositivos y las funcionalidades TIC a la valoración de los potenciales usuarios, de acuerdo a sus propios criterios de uso. 


\section{Metodología: Talleres de Participación para Conocer las Preferencias de los Potenciales Usuarios}

Para la consecución del objetivo propuesto en esta investigación es preciso alcanzar los objetivos específicos arriba señalados. Estos son, por lo tanto, los que han definido claramente las partes en las que está dividida la investigación y la metodología utilizada para la realización de este trabajo:

1. Búsqueda de información sobre prestaciones y características de los dispositivos TIC existentes en el mercado y preselección de aquellos que pueden ajustarse mejor a las necesidades de los potenciales usuarios. Se ha realizado una revisión a través de páginas web especializadas.

2. Revisión de la literatura y búsqueda de información sobre las necesidades que afectan de manera más perentoria el día a día de los mayores en el medio rural y de cuáles son las funcionalidades de las TIC que podrían servir para hacerles frente. Esta etapa se ha centrado en informes e investigaciones académicas.

3. Valoración, por parte de los potenciales usuarios, de los dispositivos y las funcionalidades TIC, de acuerdo a sus propios criterios de uso. En esta etapa se ha trabajado con personas mayores del medio rural y por tanto potenciales usuarios. Se han realizado talleres de participación en distintos municipios rurales, que han permitido explicar y mostrar las funcionalidades de las nuevas tecnologías. Se han desarrollado once talleres, con una asistencia total de 122 personas mayores. Las sesiones se han realizado con una media de 11 personas, a las que se les han mostrado los dispositivos y las funcionalidades para mejorar su calidad de vida, y se les ha pedido que las valoraran a través de un cuestionario, con el fin de determinar cuáles son sus preferencias al respecto.

Los resultados de estos talleres es donde radica el interés de esta investigación, ya que aportan información de primera mano sobre cómo es la relación que se establece entre los mayores y las TIC. Para realizar este análisis se ha utilizado, por un lado, una hoja de valoración que ha permitido una apreciación más cuantitativa de los dispositivos y las funcionalidades evaluadas (resultados interpretados mediante el programa de análisis estadístico SPSS 15.0); por el otro, una observación más cualitativa de la 
predisposición y las actitudes mostradas por los participantes a lo largo de dichos talleres.

\section{Resultados: Análisis y Selección de Dispositivos y Funcionalidades TIC para Resolver Necesidades de los Mayores en el Medio Rural}

Para realizar el análisis y la selección de dispositivos y funcionalidades TIC, se debe hacer en primer lugar una aproximación a este complejo y mudable mundo, tomando en consideración las necesidades específicas del colectivo de mayores en el medio rural.

\section{Dispositivos TIC Ajustados a las Necesidades de los Mayores}

Las tecnologías de la información y la comunicación se conciben como "dispositivos tecnológicos (hardware y software) que permiten editar, producir, almacenar, intercambiar y transmitir datos entre diferentes sistemas de información que cuentan con protocolos comunes. Estas aplicaciones, que integran medios de informática, telecomunicaciones y redes, posibilitan tanto la comunicación y colaboración interpersonal (persona a persona) como la multidireccional (uno a muchos o muchos a muchos). Estas herramientas desempeñan un papel sustantivo en la generación, intercambio, difusión, gestión y acceso al conocimiento" (Cobo, 2009).

Su rápida evolución ha dado lugar a nuevos paradigmas que permiten establecer nuevas formas de vida y relación social. Entre otros conceptos, se puede resaltar el concepto "Internet de las cosas" (Internet of Things, IoT), (Atzori et al., 2010) en el que diferentes objetos cotidianos permiten recoger y mandar información a través de variadas tecnologías con posibilidad de personalización, sirviendo, de este modo, de apoyo a los ciudadanos en su día a día.

Se trata de replantear y perfeccionar constantemente los dispositivos que permitirán a los ciudadanos mejorar su calidad de vida. En concreto, toman especial protagonismo los "pequeños dispositivos" (smartdevices) o dispositivos con capacidad de procesamiento propio y de comunicación con otros dispositivos de su entorno. Dentro de este tipo destacan los "dispositivos móviles" (mobiledevices), caracterizados por ser portables y 
generalmente permitir el acceso a datos e información desde cualquier lugar. Hoy en día, gracias a los avances tecnológicos, también es posible que prácticamente todos los dispositivos móviles puedan conectarse sin cables a una red, permitiendo integrar en un mismo dispositivo multitud de aplicaciones que han ampliado las funcionalidades ofrecidas a los usuarios. Así pues, algunas de las características que en este momento definen los dispositivos móviles son (Davy, 2003; Pérez, 2012; Rouse, 2006).

- Son aparatos pequeños

- Son portables

- Tienen capacidad de procesamiento

- Pueden conectarse a la red

- Tienen memoria limitada

Por otro lado, de forma simultánea, Internet y los teléfonos móviles pasan a ser tecnologías comunes para las personas mayores (Kurniawan, 2007, 2008; Enwald, 2016). Muchos mayores utilizan estos dispositivos en actividades de comunicación, búsqueda de información y ocio (Kurniawan, 2007), de modo que su actitud hacia ellos se va haciendo positiva e, incluso, consideran que estos servicios les ofrecen seguridad (Ling, 2008; Melander, 2008) y ayuda en sus relaciones sociales (Hill, 2015). En el caso de los teléfonos móviles esta actitud está todavía más remarcada, se han vencido reticencias y el uso del móvil se ha convertido para los mayores en una costumbre cotidiana (Oksman, 2006).

En este contexto, y considerando la visión de futuro en la que los habitantes (no solo del medio urbano sino también del entorno rural) podrán demandar multitud de aplicaciones y servicios que permitan su movilidad en la mejora de su calidad de vida, el presente estudio se ha centrado en los teléfonos móviles y las tabletas inteligentes. Estos dispositivos presentan mayor proyección y usabilidad, y se pueden usar tanto dentro como fuera de la casa. De esta forma, se está optando por dispositivos móviles que permiten a los usuarios su portabilidad sin interferencia en las actividades de su vida cotidiana.

A continuación se describirán brevemente. 


\section{Teléfonos Móviles}

Un teléfono móvil es un dispositivo electrónico de comunicación, normalmente de diseño reducido y sugerente, y basado en la tecnología de ondas de radio (es decir, transmite por radiofrecuencia), que tiene la misma funcionalidad que cualquier teléfono de línea fija. Su rasgo característico principal es que se trata de un dispositivo portable e inalámbrico, esto es, que la realización de llamadas no es dependiente de ningún terminal fijo y que no requiere de ningún tipo de cableado para llevar a cabo la conexión a la red telefónica. Además de ser capaz de realizar llamadas como cualquier otro teléfono convencional, un teléfono móvil más o menos moderno suele incorporar un conjunto de funciones adicionales, tales como mensajería instantánea (sms), agenda, juegos, etc., que aumentan la potencialidad de utilización de estos dispositivos (Basterretche, 2007).

\section{Teléfonos Inteligentes}

Los teléfonos inteligentes (smartphones en inglés) son teléfonos que soportan más funciones que un teléfono común. Entre estas suelen encontrarse la de gestor de correo electrónico y la funcionalidad completa de organizador personal, y suelen estar pensados para acceder de manera continua a Internet. Así pues, estos teléfonos combinan los conceptos de teléfono móvil y ordenadores en un único dispositivo, si bien uno de sus mayores atractivos es su simplicidad. Otro atractivo de estos dispositivos es que los usuarios pueden ampliar las características del dispositivo descargando nuevas aplicaciones mediante la conexión inalámbrica (Morillo, 2011).

\section{Tabletas}

Una tableta (tablet en inglés) es un tipo de ordenador que tiene una pantalla con la que se puede interaccionar directamente. De manera opcional puede tener accesorios como, por ejemplo, un teclado externo para facilitar el 
trabajo de sobremesa. Este dispositivo estaba pensado para trabajos de campo, como una alternativa competitiva a los portátiles.

En cuanto al tamaño, estos dispositivos son ligeramente más pequeños que los ordenadores portátiles y algunos de ellos tienen la capacidad de cambiar su apariencia (portátil o tableta), de modo que en la posición de portátil tiene un teclado para la entrada de datos, y cuando están en el formato tableta, tiene una pantalla táctil con entrada de datos basada en un lápiz (Morillo, 2011).

Tanto teléfonos inteligentes como tabletas integran agendas, bases de datos de contactos que pueden sincronizarse fácilmente con equipos de sobremesa, correo electrónico que automáticamente es recibido en el dispositivo, navegación Internet, blocs de notas, sistemas de mensajería corta, sistemas multimedia (vídeo, audio, foto, etc.) e incluso GPS con la posibilidad de usar estos terminales para navegación tanto en vehículos como a pie. Así mismo, estos terminales pueden usarse como dispositivos de almacenamiento portátiles, al disponer de una gran capacidad por el uso de tarjetas externas de almacenamiento (Bareño, 2011). Todo ello los hace muy interesantes a efectos de esta investigación.

\section{Funcionalidades TIC que Pueden Contribuir a Mantener la Calidad de Vida de las Personas Mayores}

La literatura revela que, para las personas mayores, la calidad de vida comprende una gama amplia de aspectos (Xavier et al., 2003; Walker, 2004; Plaza et al., 2011), incluidos el mantenimiento de la salud física y el bienestar psicosocial, las relaciones y el apoyo social, el desarrollo de la actividad, los recursos materiales y financieros, y el medio ambiente externo; aspectos que pueden garantizar su independencia, su autonomía y la percepción de seguir teniendo el control de su propia vida.

Así pues, la calidad de vida se traduce en la posibilidad de permanecer de forma independiente en sus propios hogares, donde pueden seguir manteniendo su ámbito de relaciones, su espacio de actividad y, en definitiva, su universo conocido y valorado de tantos años (García, 1997; Walker, 2004; Wiles et al., 2012).

Sin embargo, se observa cómo para los mayores la calidad de vida se va deteriorando y van surgiendo sentimientos comunes de soledad, inseguridad, 
dependencia y miedo, que en el medio rural son más difíciles de solventar debido a la falta de población. Las funcionalidades TIC pueden convertirse en un mecanismo válido para paliar esta situación.

Así pues, se ha definido una serie de funcionalidades que a través de las tecnologías de la información y la comunicación les pueden servir para mejorar su calidad de vida:

Tabla 1.

Funcionalidades TIC para la mejora de la calidad de vida de los mayores

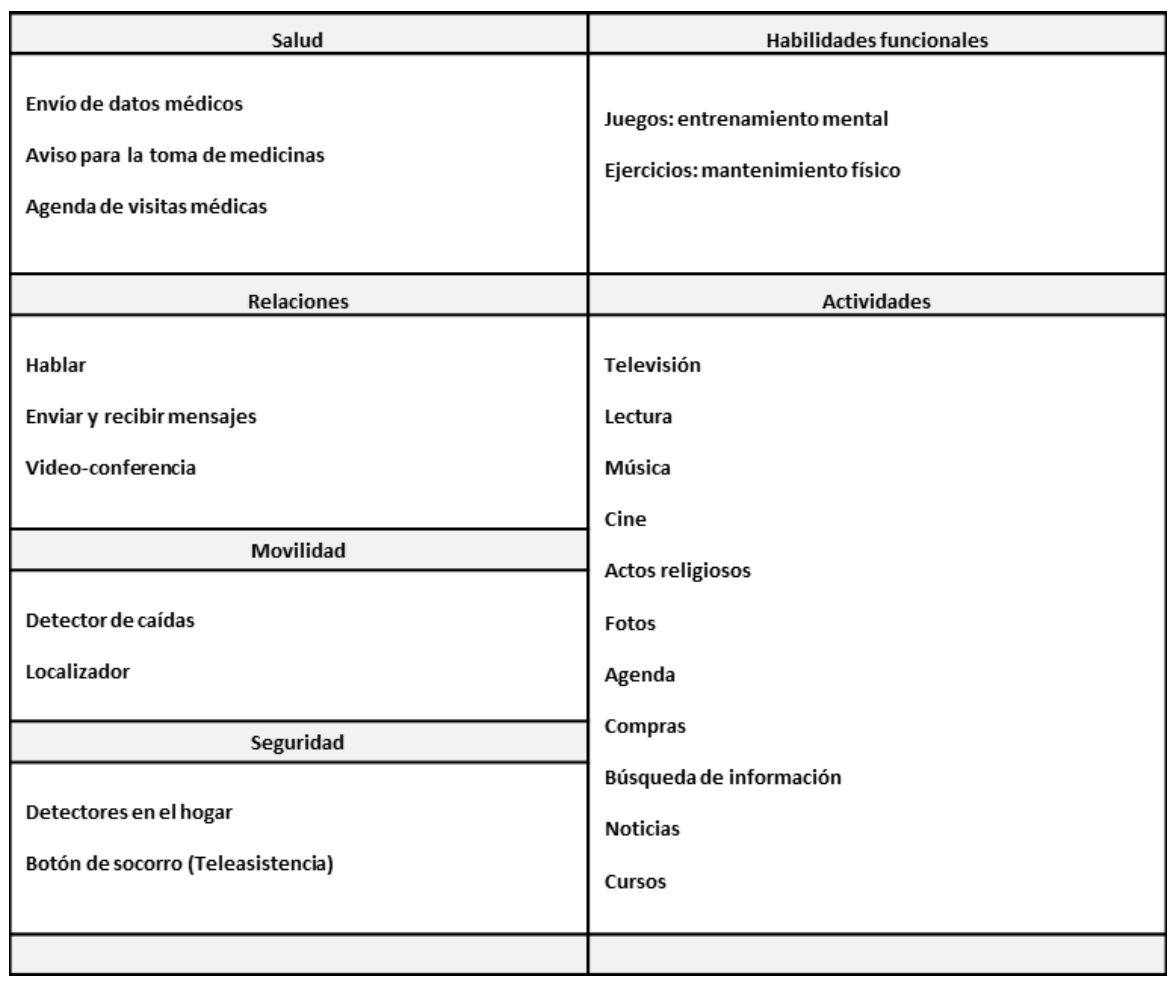

En lo concerniente a la salud, Internet puede jugar un papel importante como fuente de información y comunicación: las personas mayores pueden encontrar y compartir información (Tennant, 2015), adquirir conocimientos 
que les sirvan para la prevención de enfermedades y dependencias (Karavidas et al., 2005), incrementar una relación cercana y fluida con su médico; e, incluso, propiciar la telemedicina, esto es, el envío de datos médicos a través de dispositivos tecnológicos de forma que el profesional facultativo pueda llevar el control y el diagnóstico de su paciente de manera remota (Bertera et al., 2007; Megalingam et al., 2014); además, también puede contribuir a la correcta gestión de la enfermedad y la toma de medicación, con un seguimiento más cercano y regular del paciente (Tamburini \& Paggetti, 2005; Mitzner et al., 2010; Harrefors et al., 2010).

No obstante, la funcionalidad TIC con mejor acogida en el campo de la salud es el servicio de teleasistencia, esto es, el envío de una señal de alarma para pedir ayuda médica o indicar al centro de salud si existe algún problema (Bertera et al., 2007).

Las opiniones de los mayores, recogidas en diversos estudios, muestran cómo estos son conscientes de que las tecnologías dirigidas a la salud pueden ayudar a su independencia, haciendo posible permanecer por más tiempo en sus propios hogares (Bertera et al., 2007; Mitzner et al., 2010; Tangtisanon, 2016). Los mayores manifiestan que ciertos exámenes rutinarios de salud pueden realizarse mejor en el hogar, pues además de incrementar su comodidad, reducen la ansiedad que generan las visitas al médico (Harrefors et al., 2010). Es evidente la sensación de seguridad e independencia que las tecnologías sanitarias transmiten a sus usuarios y esto es especialmente cierto en grupos con un acceso más difícil a los servicios médicos (Bertera et al., 2007), como es el caso del colectivo objeto de estudio.

Por otro lado, en las relaciones es donde se da el uso más común de las TIC entre las personas mayores. Parece ser que la comunicación con otras personas a través de las TIC ofrece un apoyo emocional que es más difícil tener en una comunicación directa: permite un mayor contacto con familiares y amigos (Abascal \& Civit, 2001; Thayer \& Ray, 2006; Ling, 2008), especialmente con los nietos, hace frente a la ansiedad y la soledad (Karavidas et al., 2005; Sum et al., 2008), sobre todo de aquellas personas con movilidad reducida o distantes de sus seres queridos (Wagner et al., 2010). Las diferentes formas de comunicación utilizadas son correo electrónico, mensajería instantánea, WhatsApp y foros en línea, aunque cada 
una de ellas es utilizada según el grupo social con quien se interactúe (Xie, 2008; Muntaner et al., 2015).

Por último, las funcionalidades TIC también responden a la necesidad de actividad que todavía siguen teniendo las personas mayores. Internet es el primer medio digital que, de forma interactiva, se utiliza para muchos propósitos; tiene funciones tales como informar (Hill et al., 2008; Mitzner et al., 2010), formar (Boz \& Aksoy, 2011), entretener (Ijsselsteijn et al., 2007), fomentar la espiritualidad (Bell, 2006), ayudar a observar los hechos externos, y también publicitar y vender bienes y servicios (Boz \& Aksoy, 2011). Las TIC pueden convertirse en una herramienta para envejecimiento activo (Sánchez-Valle, 2017; García \& Bermejo, 2004), pues no solo mantienen su interés por seguir integrados en la sociedad en la que viven y contribuyen a su desarrollo personal y de capacidades mentales (Gaßner \& Conrad, 2010), sino que incrementan su autoestima y satisfacción personal al desenvolverse con eficacia con dispositivos de última tecnología (Karavidas et al., 2005).

Del mismo modo, las funcionalidades TIC también pueden ser de utilidad para actividades que exigen seguridad. Muchas personas mayores experimentan restricciones motoras que pueden llevarlas a posibles situaciones de riesgo, sobre todo cuando estas personas tratan de llevar una vida independiente. En consecuencia, situaciones de enfermedad, accidentes en el hogar, y otras circunstancias cotidianas de peligro pueden abordarse a través de un canal de rápida comunicación para solicitar ayuda urgente (Abascal \& Civit, 2001). Sensores en el hogar (Demiris et al., 2004) o en dispositivos móviles, sistemas de televigilancia (Scanaill et al., 2006), y aplicaciones con GPS (Boulos et al., 2007) combinadas con un servicio de comunicación directa con una persona de referencia permiten una movilidad más independiente, tanto dentro como fuera del hogar, unida a una sensación de mayor tranquilidad y seguridad en sus movimientos.

\section{Valoración: Preferencias de los Mayores respecto a los Dispositivos y las Funcionalidades TIC que Mejor Responden a sus Necesidades}

Con el fin de conocer la valoración que las personas mayores del medio rural del interior de España hacen de los dispositivos y las funcionalidades TIC 
como medios para mejorar su calidad de vida, se les ha convocado a unos talleres en los que se les han mostrado y ellos han podido observar $\mathrm{y}$ manejar.

Es preciso tener en cuenta la dificultad de establecer un perfil de persona mayor, puesto que las características que las definen son incluso más diversas que las de la población más joven, dado el amplia variedad de grados de capacidad, derivados de los distintos estadios en los procesos propios de enfermedad o envejecimiento (Eisma et al., 2004). No obstante, pese a que el único factor común en este grupo es la edad, esta sí aporta una serie de características que permiten considerarlo como un colectivo objeto de estudio.

La pérdida de capacidades físicas y cognitivas es un hecho cada vez más acentuado en este grupo de personas. En el proceso de envejecimiento se ven mermados sentidos como la vista, el oído y el tacto, así como las habilidades de atención, aprendizaje y memoria, que son importantes en el uso de dispositivos tecnológicos. Esta es, por tanto, una de las razones que explican el rechazo que se ha venido observando en las personas mayores ante el uso de dispositivos móviles.

Añadidos a estos, existen otros factores que han provocado este rechazo, resumidos en el Libro Blanco para el diseño de tecnología móvil accesible y fácil de usar (ONCE, 2011):

- Barreras mentales. Son aquellas producidas por el temor a lo desconocido. Las propias personas juzgan los dispositivos móviles e Internet como algo complicado y donde se encuentran fuera de lugar.

- Barreras físicas. Se originan en los diversos atributos y características físicas que posee el dispositivo. La forma, el tamaño, el peso o la distribución de los elementos del interfaz físico del dispositivo son parámetros que afectan a la accesibilidad del usuario. Estas características presentan dificultades a los mayores, a la hora de interactuar con los dispositivos, ya que en algunos casos pueden tener deficiencias visuales, auditivas o de movilidad.

- Barreras económicas. El alto precio de algunos dispositivos móviles y las cuotas mensuales de conexión a Internet suponen un importante obstáculo para este perfil de usuarios, ya que en la mayoría de los casos dependen de pensiones muy ajustadas. 
- Barreras de accesibilidad software. Se originan en la forma de uso definido para el dispositivo y los diversos canales de comunicación entre el dispositivo y el usuario. Estas barreras son más difíciles de identificar, ya que el software de un dispositivo afecta tanto al interfaz de control como al uso apropiado del dispositivo.

Por todo esto, y porque en el medio rural es mayor el desconocimiento que, en general, las personas mayores del medio rural tienen de estas tecnologías ${ }^{2}$ (IMSERSO, 2015), el trabajo de campo ha merecido mayor atención. Llegar a conocer la valoración que estas personas hacen sobre el papel que podrían jugar las TIC de cara a mejorar su calidad de vida ha exigido una especial labor pedagógica.

Se han llevado a cabo 11 talleres en distintos municipios del medio rural, con una asistencia total de 122 personas. Los siguientes gráficos muestran cómo la participación de mujeres ha sido significativa, un $78 \%$ del total, mientras que las distintas edades han estado representadas de una forma más igualitaria.

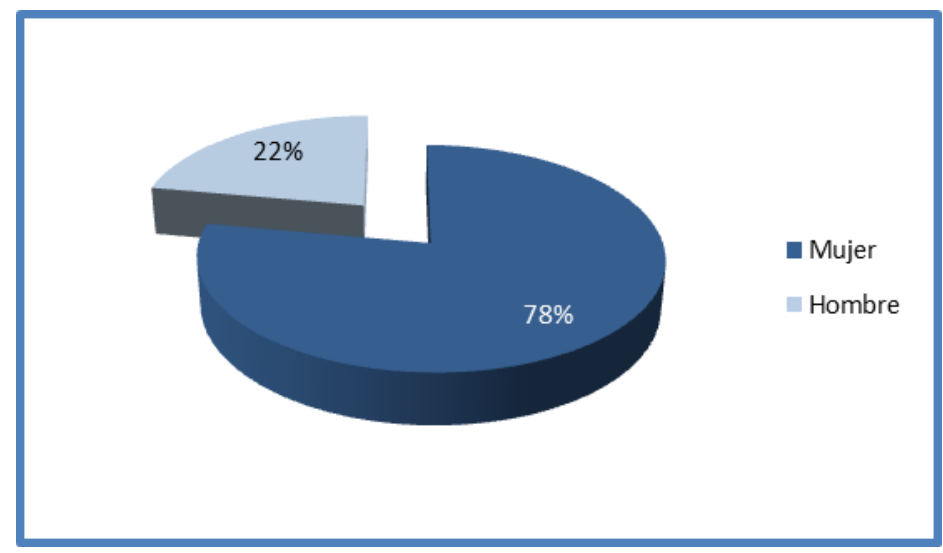

Gráfico 1. Perfil de los asistentes según sexo 


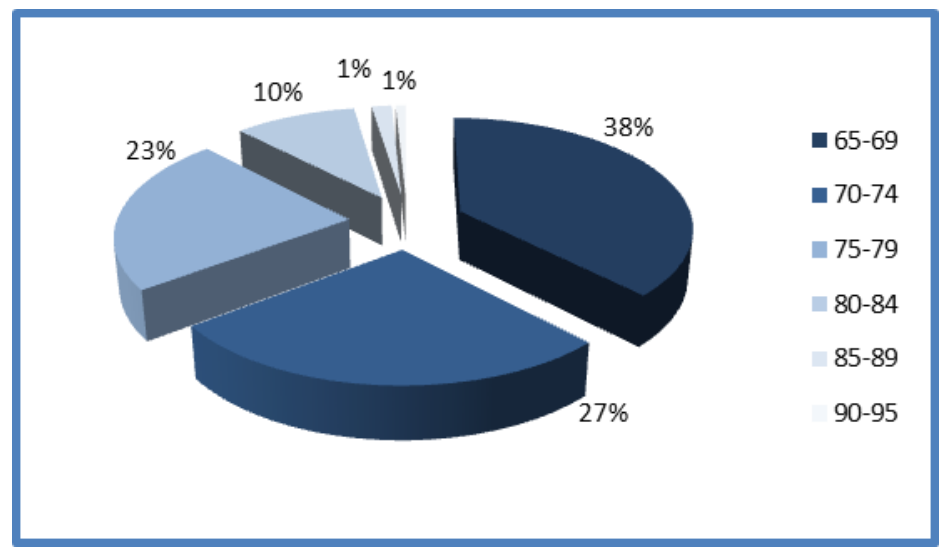

Gráfico 2. Perfil de los asistentes según edad

\section{Valoración de los dispositivos TIC}

Las sesiones de trabajo se han planteado bajo la necesidad de mostrar, en primer lugar, los dispositivos que conforman el hardware para, más tarde, exponer detenidamente las funcionalidades que estos dispositivos son capaces de realizar, mejorando su día a día. Para ello se ha llevado una pantalla de televisión, un equipo PC de sobremesa completo, un ordenador portátil, una tableta de 10 pulgadas, un teléfono inteligente y un teléfono móvil tradicional, de modo que pudieran verlos, manejarlos y comprender más fácilmente las características, utilidades y formas de uso que se exponían de cada uno de ellos, haciendo especial hincapié en las posibilidades de uso dentro y fuera del hogar. Con algunos de los equipos ya estaban más familiarizados, mientras que era la primera vez que veían y tocaban otros, de modo que esta ha sido una buena oportunidad para mostrárselos y, sobre todo, para romper con el miedo preconcebido a tener grandes dificultades en su uso.

La literatura indica que las motivaciones de los usuarios hacia el uso de los dispositivos tecnológicos aumentan cuando estos resultan fáciles de utilizar, como ya se ha mencionado anteriormente, pero también cuando el dominio de la persona en el uso de la tecnología ha aumentado con la 
experimentación, es decir, cuando la persona ha utilizado anteriormente un dispositivo similar, que ya ha tenido oportunidad de manejar e interactuar con él y, por lo tanto, le resulta más familiar (Conci, 2007).

Es precisamente por este motivo por el que también es relevante conocer cuántas de las personas asistentes habían utilizado anteriormente teléfono móvil y cuántas habían utilizado Internet. A pesar de que el teléfono móvil está ya muy introducido en nuestra sociedad, todavía existe un sector de la población (más numeroso entre las personas mayores) que no lo ha utilizado nunca. Tal y como se muestra en el gráfico 3, entre los asistentes a los talleres, el porcentaje, un $17 \%$, de personas mayores que no han utilizado nunca el móvil no es muy elevado; quizás pueda deberse a que, como ya se ha visto más arriba, la mayor parte de los asistentes tenía edades comprendidas entre 65 y 74 años (los más jóvenes dentro del colectivo contemplado).

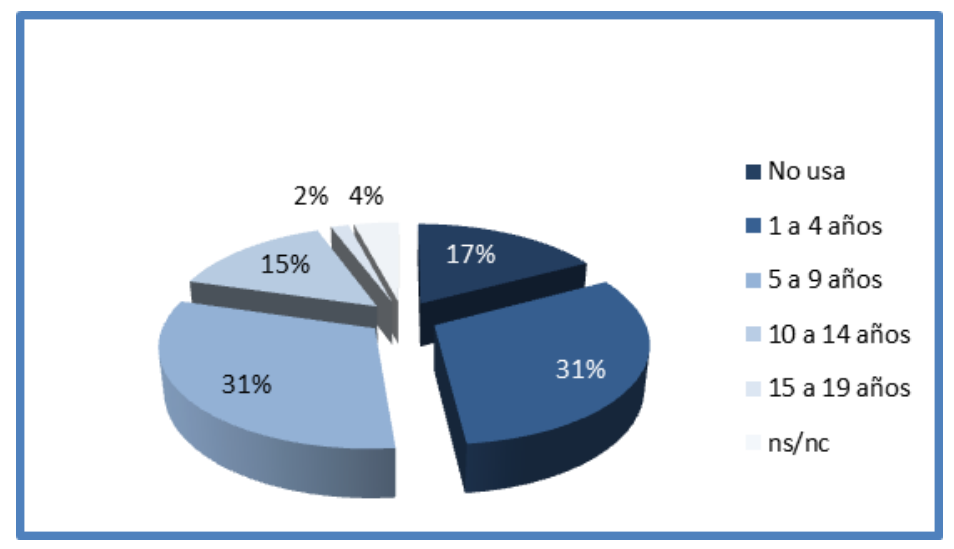

Gráfico 3. Perfil de los asistentes según el uso anterior del móvil

Sin embargo, no ocurre lo mismo respecto al uso de Internet (ver gráfico 4). Pese a que en general, en España, se está observando cómo Internet comienza a formar parte de la vida de los mayores (Fundación Telefónica, 2016), no ocurre lo mismo con los asistentes a los talleres. En este caso, el porcentaje de personas que no han usado con anterioridad Internet, el 71\%, es significativamente elevado. 


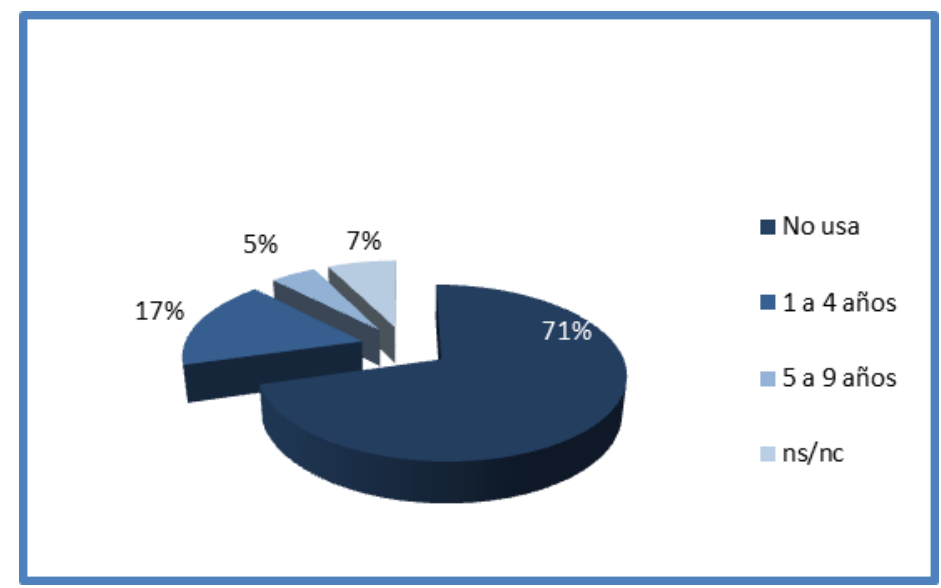

Gráfico 4. Perfil de los asistentes según el uso anterior de Internet

La dinámica de los talleres ha sido sencilla: se ha ido explicando y mostrando los distintos dispositivos móviles, haciendo hincapié en las características que según la literatura se consideran importantes: tamaño, forma, manejo, color, y precio. Para ello se ha utilizado una presentación proyectada sobre una pantalla, para que las imágenes ilustraran lo que se les estaba explicando; así como dos teléfonos inteligentes y una tableta, que los asistentes podían observar y tocar para familiarizarse con su uso; luego se les proporcionaba una hoja de valoración, donde podían expresar sus preferencias. 


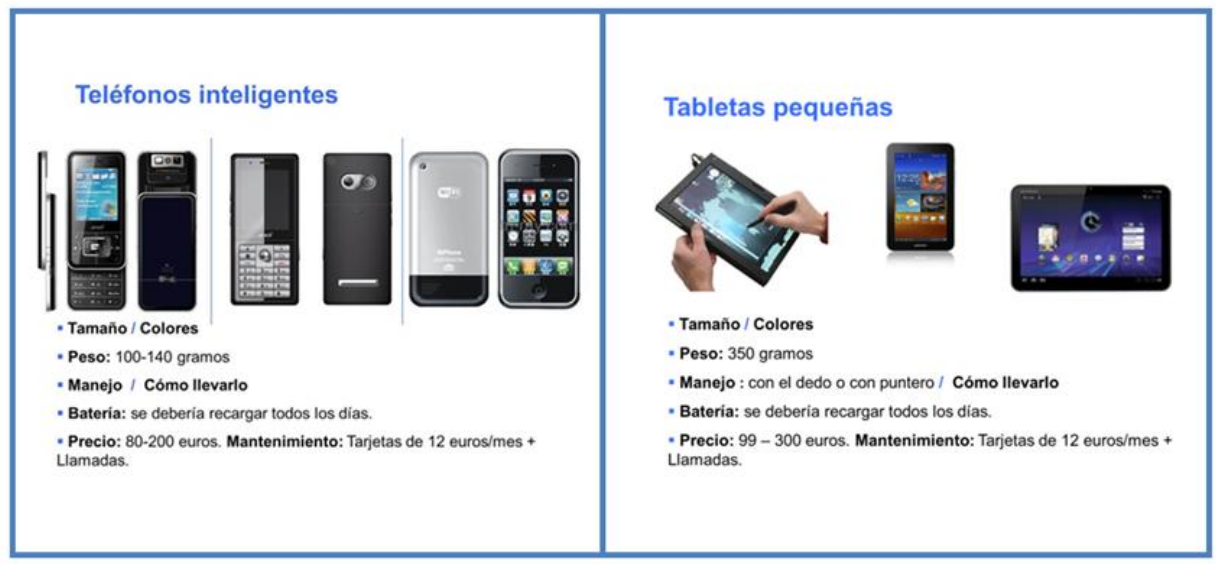

Imagen 1. Ejemplo de diapositiva expuesta en las sesiones

Pese a que la literatura indica que los mayores prefieren teléfonos que sean grandes, porque eso facilita su manejo, es llamativa la respuesta observada en los talleres. Aquí se muestra una clara preferencia por el teléfono móvil pequeño, en segundo lugar, se elige el móvil grande, y, por último, la tableta pequeña (ver gráfico 5).

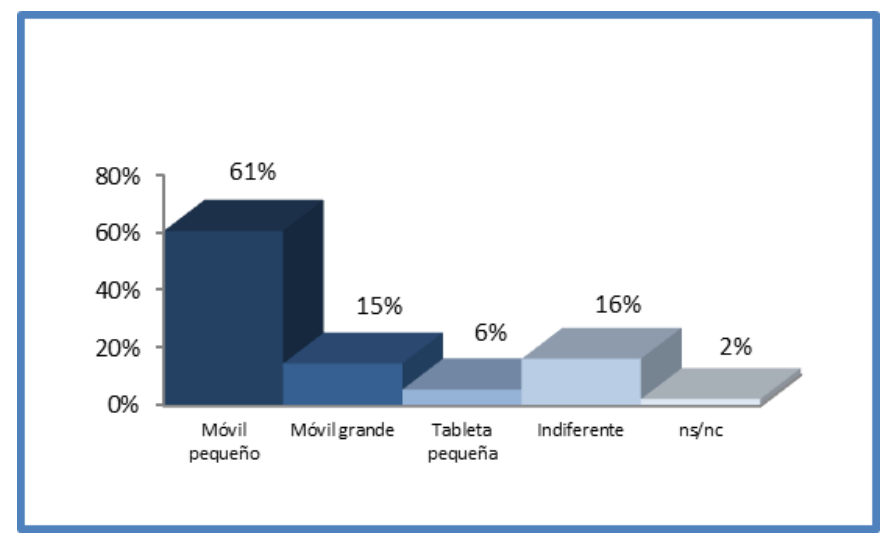

Gráfico 5. Elección en función del tamaño

Teniendo en cuenta la forma del dispositivo, esto es, si este tiene o no tapa, también ha sido elevada la inclinación hacia aquellos dispositivos que 
cuentan con la protección de la tapa, tal y como se refleja en el gráfico 6 . Analizando los datos, no se ha visto que la respuesta haya variado según el sexo de los participantes.

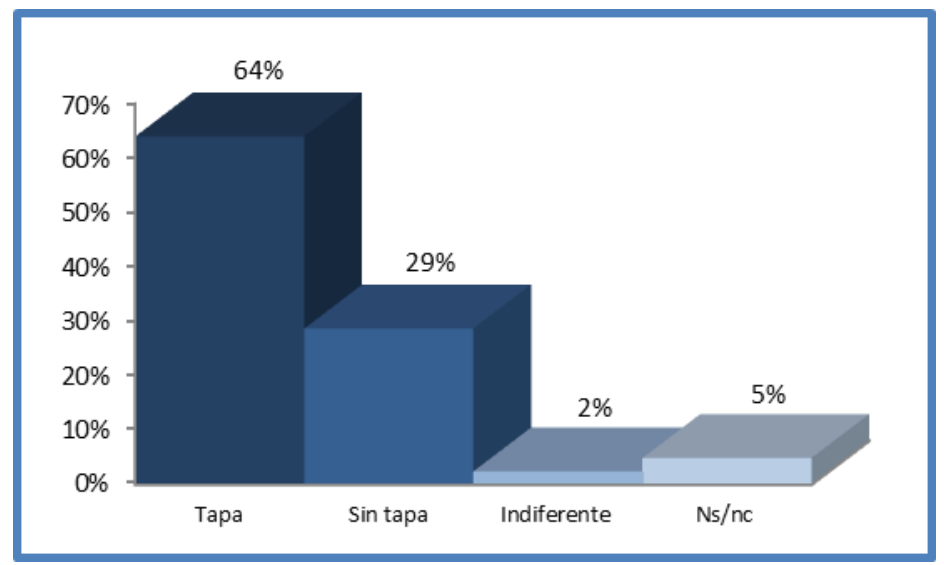

Gráfico 6. Elección en función de la tapa

En cuanto al manejo, los asistentes a los talleres han mostrado también gran coincidencia al elegir, en mayor media, el teclado frente a la pantalla táctil, un $70 \%$ frente a un $26 \%$, tal y como se muestra en el gráfico 7 . Este resultado se considera coherente, si se tiene en cuenta que los mayores están más familiarizados con el teclado de los teléfonos tradicionales. Además, la pantalla táctil representa una novedad y, a priori, les parece más difícil de utilizar. 


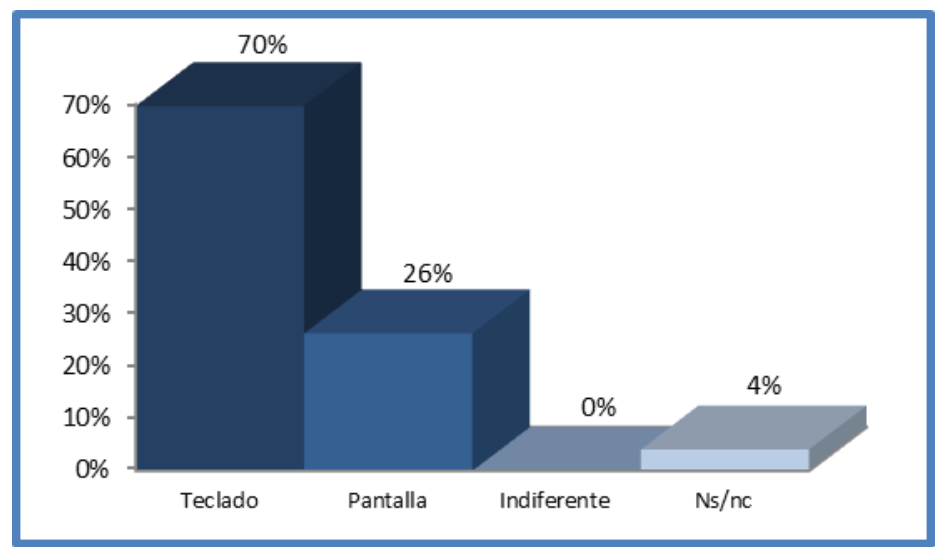

Gráfico 7. Elección según el manejo

Como ya se ha comentado más arriba, el color es una característica también diferenciadora. Como se desprende de los datos del gráfico 8, existe una clara inclinación hacia colores discretos como el negro o el gris o bien un sentimiento de indiferencia ante esta característica. Estos datos coinciden parcialmente con los encontrados en estudios previos, en los que indicaba que las mujeres habían optado por el color oro o el color plata ya que los consideraban mejores para facilitar la localización del dispositivo, mientras que los hombres mostraban indiferencia frente al color (Kurniawan, 2007). Sin embargo, en los resultados obtenidos en este estudio el $78 \%$ de los entrevistados eran mujeres (ver gráfico 1) y se obtiene el mismo porcentaje de personas que se muestran indiferentes que el que prefiere colores discretos (negro, gris). Por otro lado, si la explicación radica en la facilidad para localizar el dispositivo, deberían haber elegido colores más llamativos. 


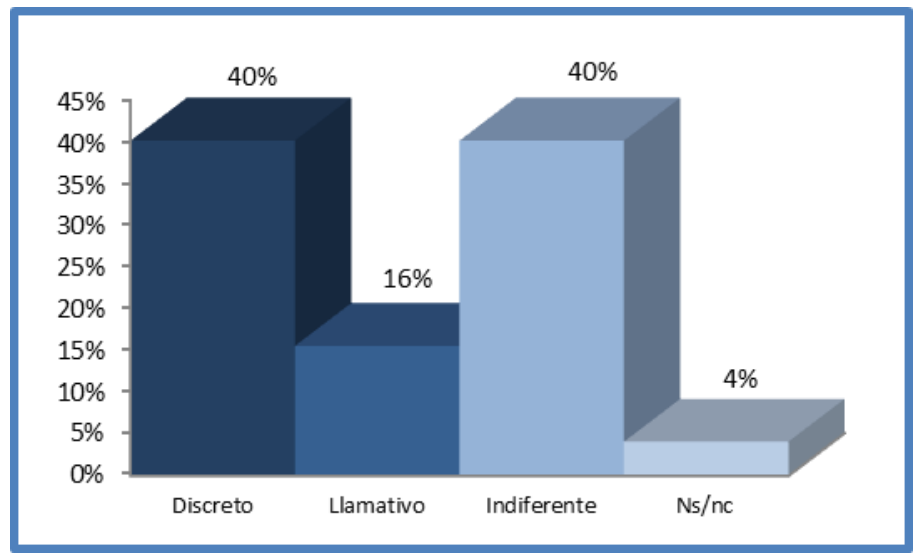

Gráfico 8. Elección según el color

La elección del dispositivo móvil según el precio de adquisición del aparato y el precio mensual de mantenimiento también ha resultado bastante evidente. El 82\% de los asistentes ha preferido el teléfono inteligente (ver gráfico 9). Esto puede estar motivado porque se les ha comentado que algunas de las compañías telefónicas regalan el teléfono inteligente dentro de sus campañas de fidelización, pero no las tabletas. Además, en global, el precio de las tabletas es superior al de los teléfonos inteligentes si bien el mantenimiento posterior cuesta lo mismo. En este punto, también es importante señalar que la variable económica es de enorme relevancia para las personas mayores del medio rural, por su marcada propensión al ahorro, pero también porque en su gran mayoría perciben pensiones propias del régimen especial agrario, que no llegan a los 600 euros mensuales, lo que les obliga a ser cuidadosos con su gasto. 
160 Rubio, Plaza \& Orive - Soluciones TIC para Personas Mayores

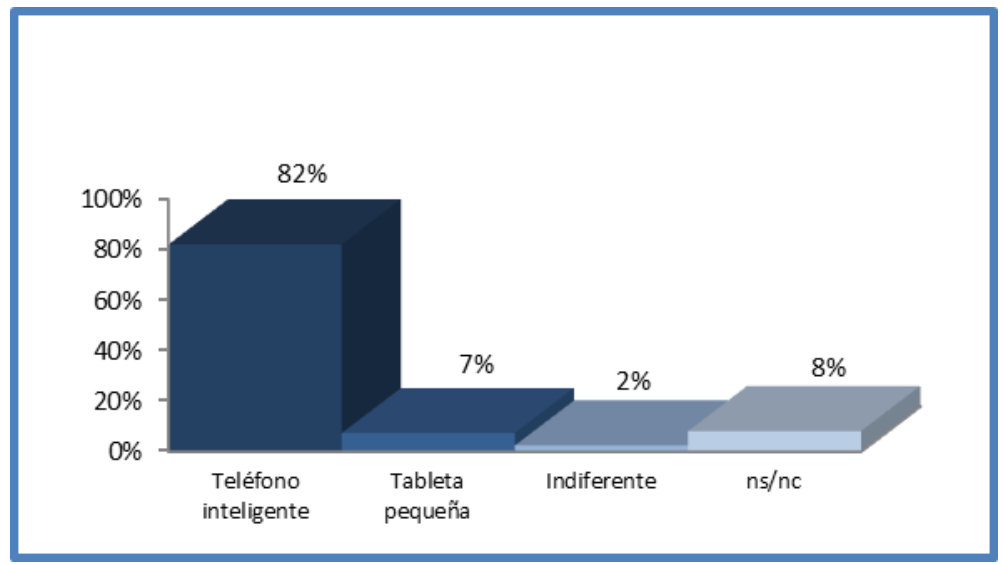

Gráfico 9. Elección según el precio

Como conclusión final, y teniendo en cuenta todas las características de los dispositivos móviles mostradas a las personas mayores, asistentes a los talleres, la elección del teléfono móvil inteligente ha sido incontestable, con un $78 \%$ frente a un $11 \%$ que ha elegido las tabletas.

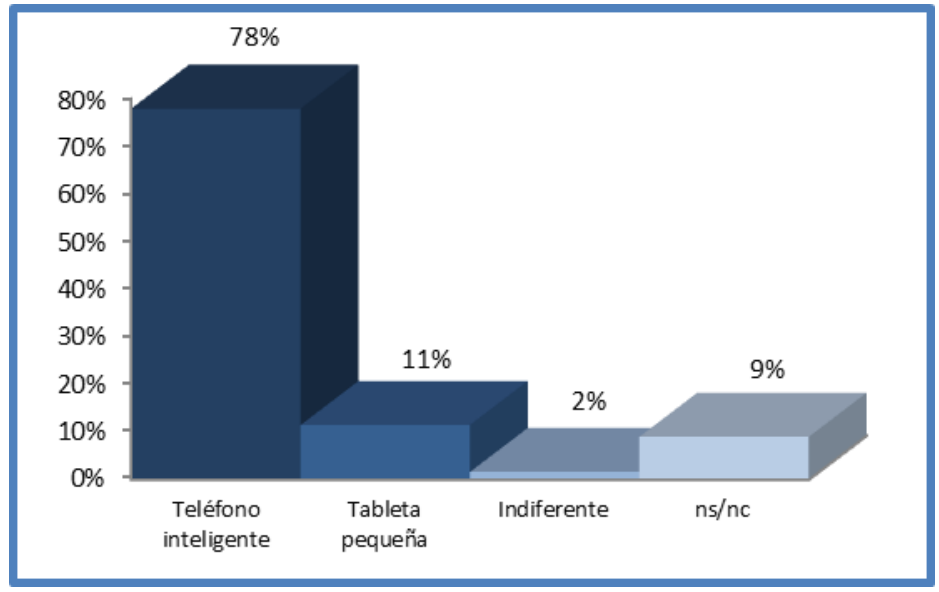

Gráfico 10. Elección del dispositivo por parte de los asistentes 


\section{Valoración de las funcionalidades TIC}

La asistencia a los talleres también ha servido para que los mayores valoraran las funcionalidades que podían desempeñar las TIC en los distintos ámbitos de su desarrollo cotidiano, ya señalados anteriormente: salud, habilidades funcionales, actividades, relaciones, movilidad y seguridad.

Para su mejor comprensión se ha explicado cada función a través de diapositivas proyectadas en una pantalla, con un lenguaje sencillo, imágenes ilustrativas y ejemplos claros que les resultaran cercanos, permitiendo toda clase de interrupciones para preguntar, comentar o pedir la aclaración de algún concepto no entendido. Hay que destacar la excelente aceptación reflejada por los asistentes, cuando se les explicaba en qué consistía cada función y en qué ámbito de su día a día les podía resultar de utilidad. Se han mostrado muy abiertos a las funcionalidades propuestas y en ningún momento se han escuchado comentarios negativos.

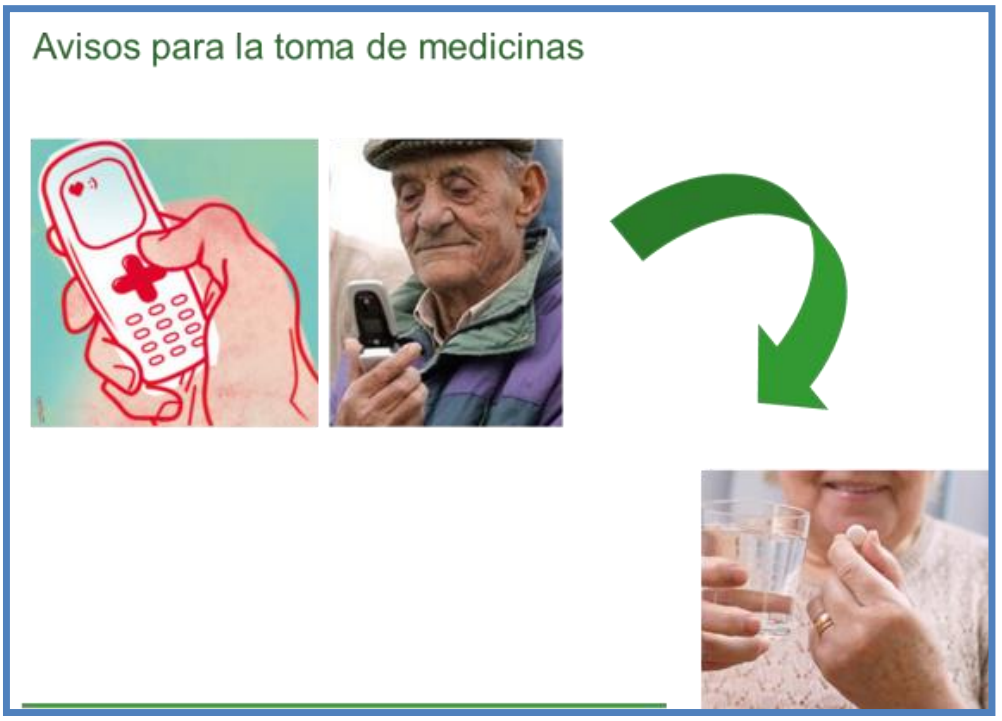

Imagen 2. Ejemplo de diapositiva expuesta en las sesiones

Para cuantificar el grado de aceptación de estas funcionalidades, se les ha pedido que las valoraran a través de una escala de medida adaptada, con tan 
solo cuatro valores, de modo que les resultara fácil contestar: nada, poco, bastante y mucho. Posteriormente, se ha transformado en una escala numérica, del 1 al 4, más fácil de analizar cuantitativamente por el equipo investigador. Todo ello ha quedado recogido en una hoja de valoración, facilitada a cada uno de los asistentes.

Así pues, los resultados obtenidos en este proceso se muestran alejados de ese prejuicio que sostiene que existe reticencia al uso de las TIC por parte de las personas mayores. Los asistentes, en general, han valorado todas las funcionalidades de forma positiva, como se observa en el gráfico 11. Se han mostrado muy receptivos a estas nuevas formas de dar respuesta a sus problemas cotidianos: 


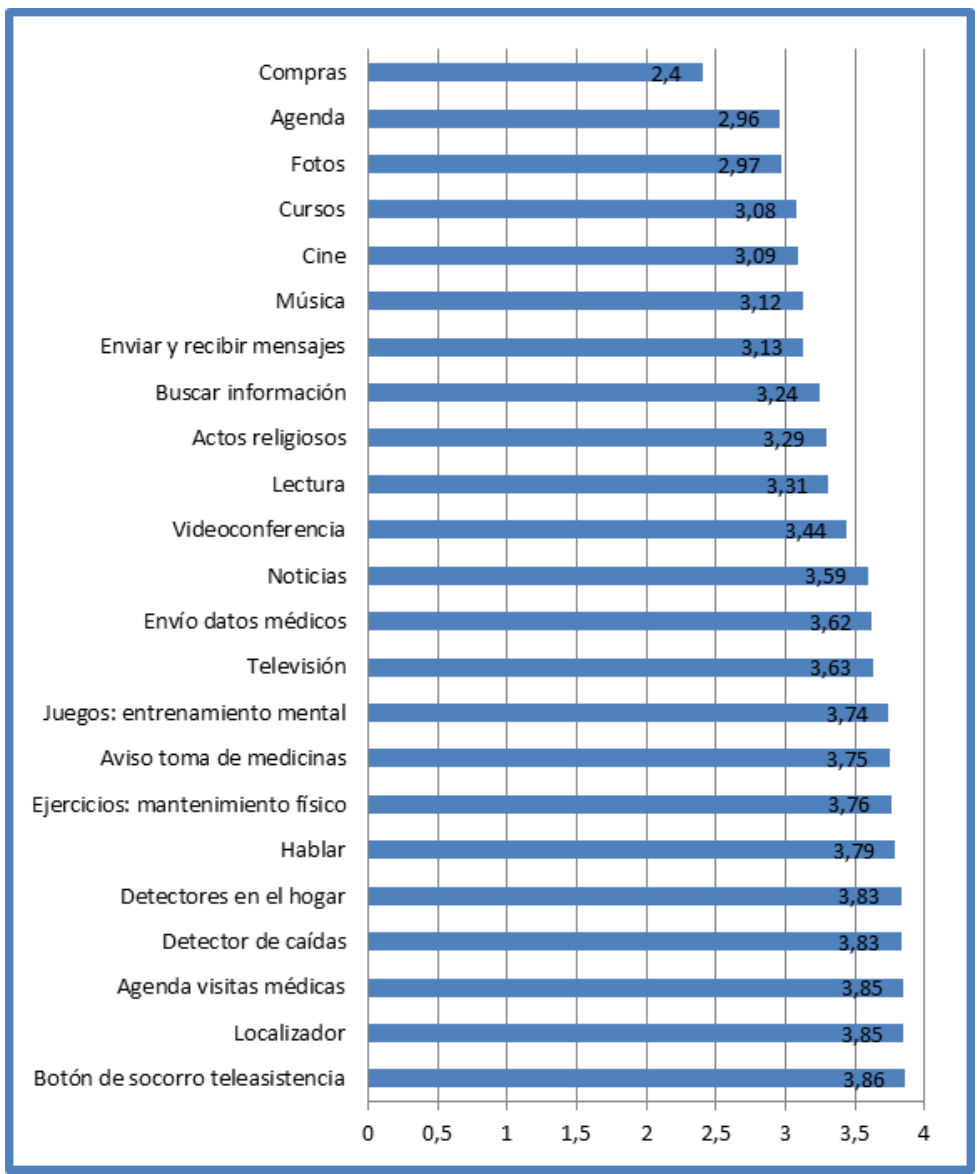

Gráfico 11. Valoración de las funcionalidades TIC (puntuaciones medias)

Analizando los datos anteriores, es preciso advertir que las funcionalidades con valoraciones más altas son precisamente aquellas para las que las personas mayores del medio rural no ven otra forma posible de cubrir sus necesidades. Son, por tanto, funcionalidades que han tenido una acogida muy positiva, pues se perciben como una opción real a los problemas que aquejan a los mayores en un entorno de fuerte despoblación, donde no hay personas cercanas que puedan prestarles una atención continuada. 
Atendiendo al sexo de los asistentes, en el siguiente gráfico se puede observar cómo los hombres han dado, en general, valoraciones más elevadas a las funcionalidades TIC, quizás debido a que ellos pueden tener una relación anterior más cercana con las tecnologías, o pueden tener mayor predisposición inicial.

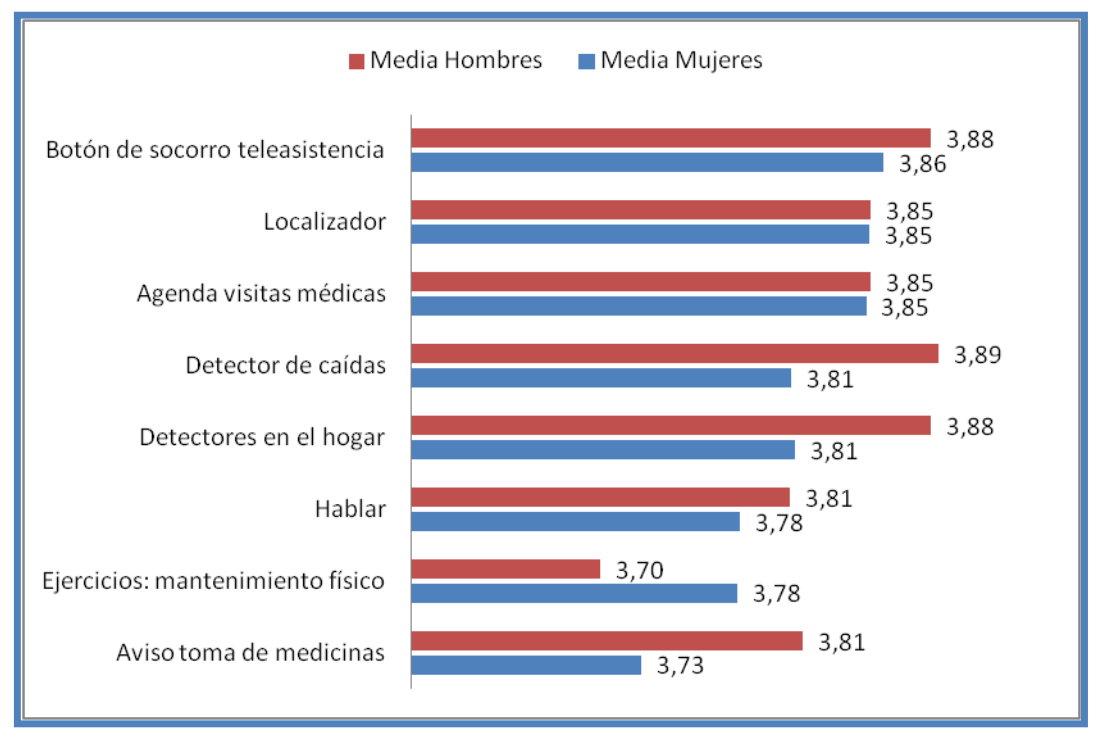

Gráfico 12. Funcionalidades más valoradas, según el sexo (puntuaciones medias)

Si se tiene en cuenta la edad de los asistentes, resulta llamativo observar cómo son los más mayores, es decir, de 85 años en adelante, quienes se revelan más favorables a estas funcionalidades. Hay que advertir que este ha sido el colectivo menos numeroso en las sesiones de trabajo, pero también el que comienza a perder autonomía en su día a día y muestra mayor dependencia de las personas cercanas. A las personas mayores les duele molestar, no quieren convertirse en una carga para aquellos que les rodean y, por esta razón, pueden ver en las funcionalidades TIC un instrumento muy valioso para aliviar esta carga "Mira, así mi hija no tendría que estar tan pendiente de mí". A pesar de que, como ya se ha comentado anteriormente, la relación previa de los mayores con las TIC no ha sido muy usual, ellos 
confían en que sus hijos o nietos les ayuden en el manejo de estos dispositivos y así logren devolverles cierta independencia. Algún asistente ha comentado con orgullo, cómo se comunica con su nieto a través de Skype "Ah, pues esas son las llamadas que yo hago con mi nieto"; su beneficio era doble, poder comunicarse a través de la imagen en pantalla, pero también sentirse más cercano al chico a través de una herramienta que este le había propuesto y enseñado a utilizar.

Gráfico 13. Funcionalidades más valoradas, según la edad (puntuaciones medias)

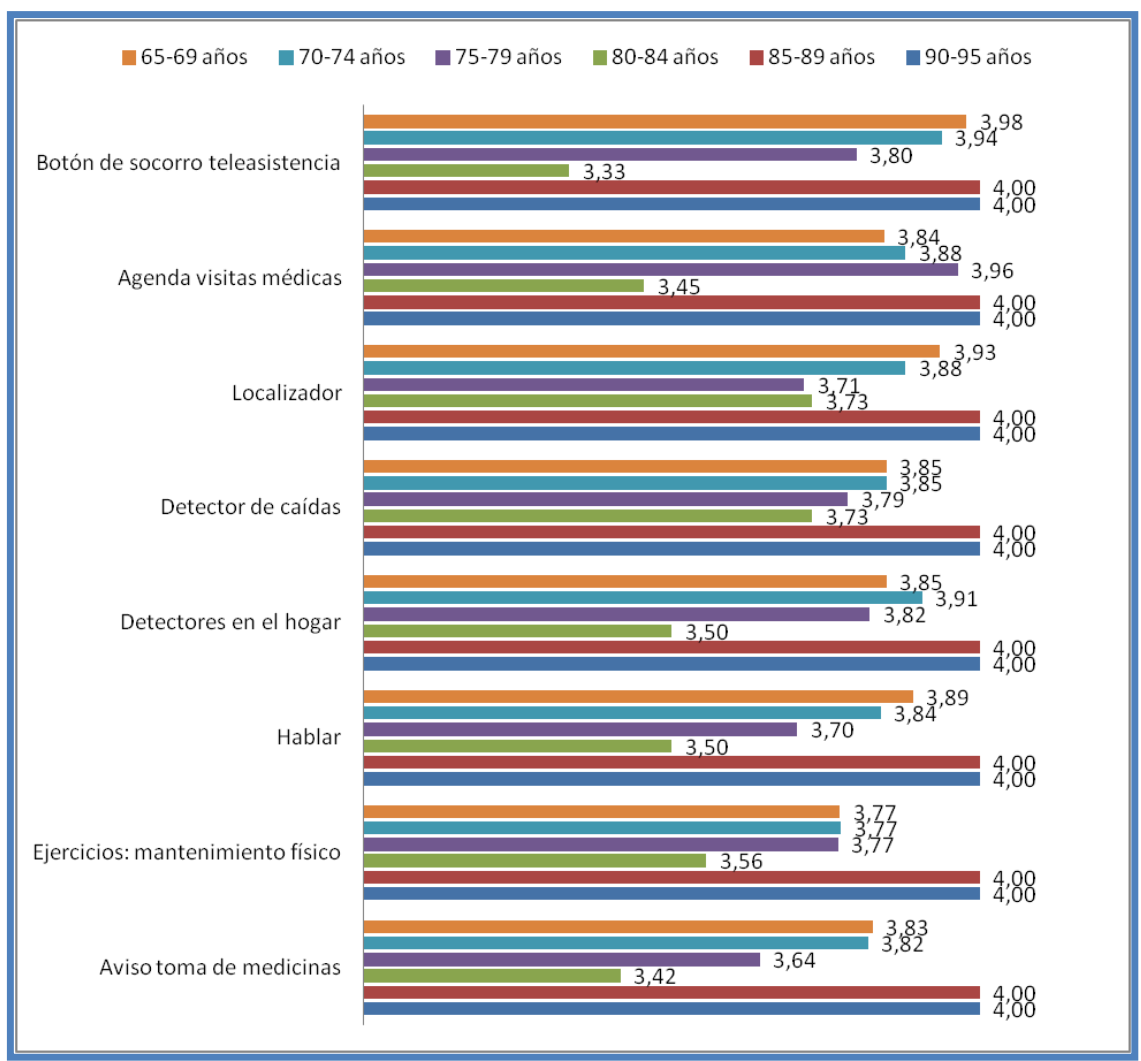

En este punto, es importante advertir cómo el uso anterior de las tecnologías de la información y la comunicación por parte de las personas mayores sí genera una predisposición positiva en la valoración de las 
funcionalidades que ahora se les presentan. Como se puede apreciar en el gráfico 14, la relación es directa, ya que prácticamente para todas las funcionalidades la valoración se incrementa conforme se incrementa el número de años de utilización del teléfono móvil, aunque ello no quiere decir que quien no lo haya usado todavía tenga una predisposición negativa. El teléfono móvil ya está muy introducido en nuestra sociedad y todas las personas mayores lo conocen, aunque no lo usen, y no les genera una percepción de temor o rechazo.

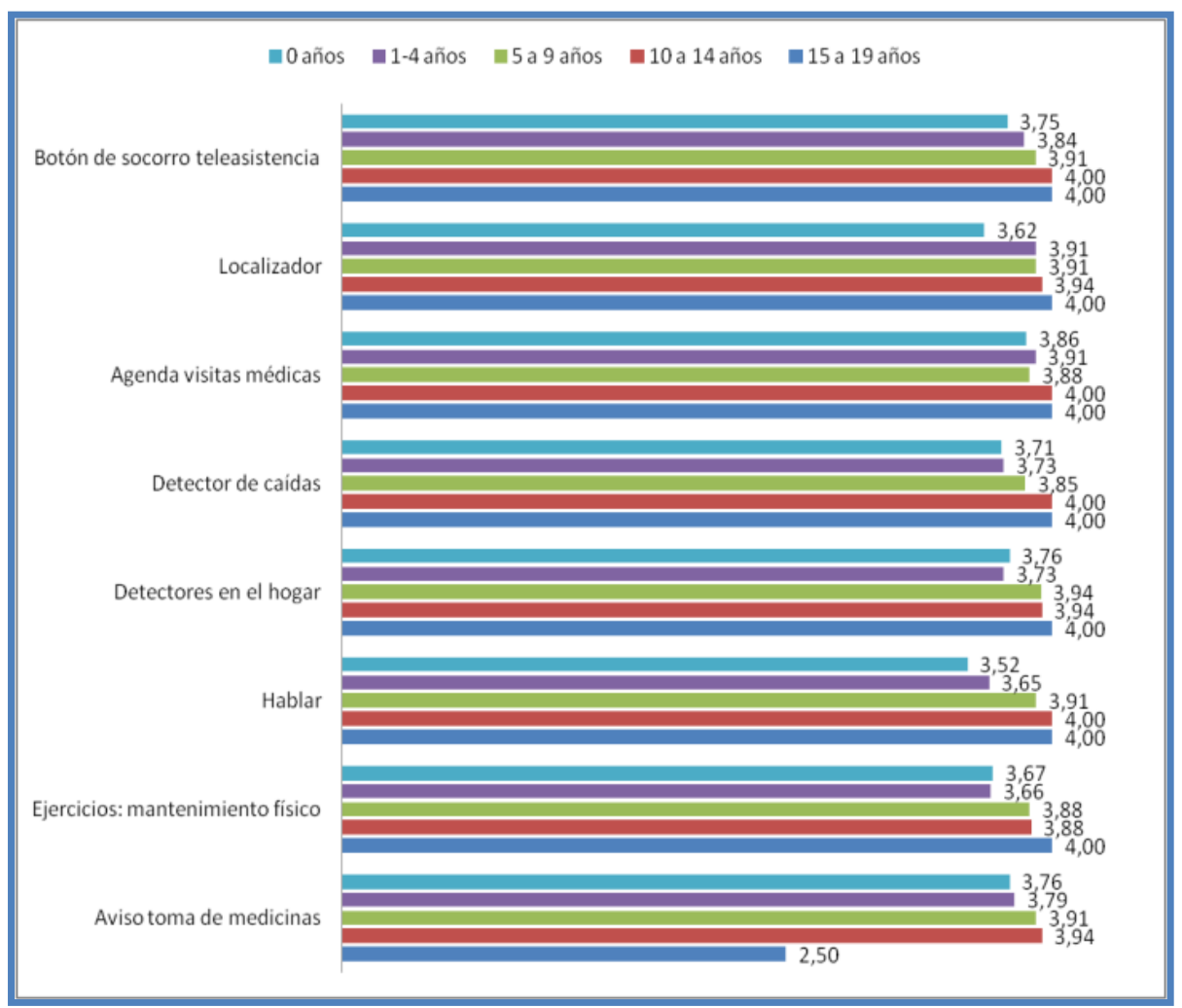

Gráfico 14. Funcionalidades más valoradas, según el tiempo de uso del móvil (puntuaciones medias) 
Sin embargo, si bien se observaba una relación positiva entre el uso del teléfono móvil y la valoración de las funcionalidades TIC por parte de los asistentes, en lo referente al uso previo de Internet no se puede afirmar lo mismo. Como muestra el gráfico 15 , no se puede decir que las valoraciones sigan una pauta marcada por la experiencia previa en el uso de Internet. Quizás esto se deba a que, tras las demostraciones en las sesiones de trabajo, a los asistentes les queda la percepción de que, en su papel de usuario, no les es necesario un conocimiento previo especial en el manejo de esta red de comunicación. Las funcionalidades que se les ha mostrado les han parecido fáciles de usar, en algunos casos ya las conocían o conocían otras similares a través de familiares, amigos o la televisión, y ello ha hecho que se mostraran más abiertos.

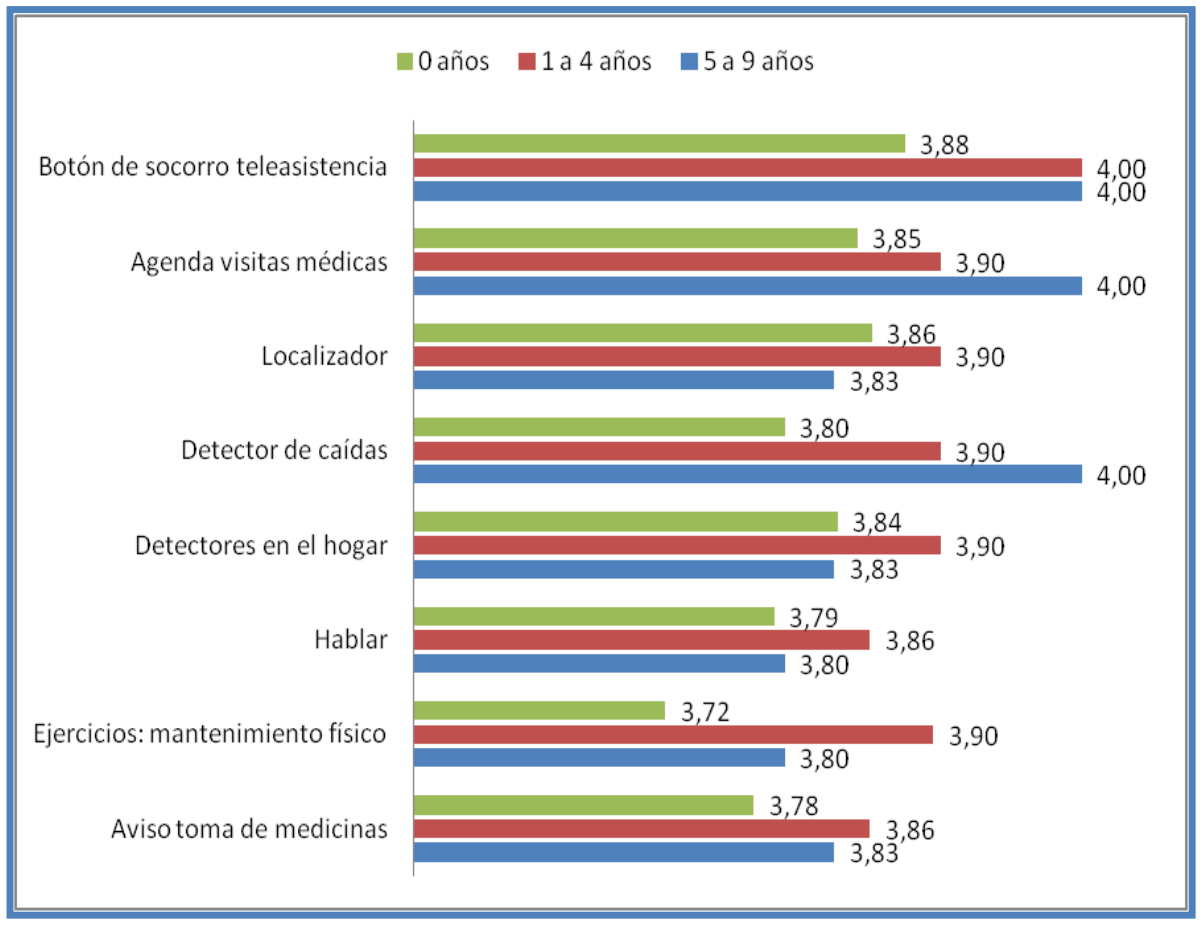

Gráfico 15. Funcionalidades más valoradas, según el tiempo de uso de Internet (puntuaciones medias) 
Así pues, es preciso destacar que, pese a que muy bajo porcentaje de las personas asistentes ha utilizado previamente Internet, la percepción ante las nuevas funcionalidades ha sido, sorprendentemente, muy positiva. Su valoración ha sido, en general, alta y han manifestado a lo largo de todas las sesiones plena confianza en poder utilizarlas y entre ellos se animaban "pues no es tan difícil, creía que me iba a costar más", "chica, pues (aprenderemos) igual que hemos aprendido a usar el microondas u otros aparatos", "si lo puedo hacer yo, ¿no lo vas a poder hacer tú?", "no parece tan difícil, aunque al principio nos han de ayudar".

En definitiva, se puede señalar que la actitud de las personas mayores asistentes ha sido muy receptiva al hecho de poder apoyarse en el uso de estas funcionalidades TIC para intentar mejorar su calidad de vida, ya que les pueden aportar mayor autonomía y seguridad. No obstante, hay que llamar la atención sobre el hecho de que la predisposición de los asistentes también ha sido de querer aprender más: estas sesiones se les han hecho cortas, les han despertado la curiosidad y estarían encantados de que se organizaran talleres que les mostraran mejor su uso. Esta voluntad de aprender y utilizar las TIC en su vida cotidiana es acorde con las conclusiones obtenidas en otros estudios (Fan, 2016).

\section{Conclusiones}

Las características del medio rural del interior de España, con elevada despoblación y escasos servicios públicos, generan unas necesidades particulares para las personas mayores que aún viven en sus pueblos. Las TIC se evidencian como una solución plausible en la respuesta a estas necesidades siempre que sus potenciales usuarios así lo manifiesten en sus preferencias.

Los avances en las TIC llevan a un replanteamiento y perfeccionamiento constantemente de los dispositivos que permiten a los ciudadanos mejorar su calidad de vida. En concreto, están tomando especial protagonismo los dispositivos móviles, caracterizados por ser portables y permitir el acceso a datos e información desde cualquier lugar. Son precisamente estos dispositivos los que se han seleccionado para esta investigación, pues, a diferencia de los ya tradicionales teléfonos móviles, la mayor parte de los 
teléfonos inteligentes y de las tabletas dispone de un sistema operativo libre, lo que permite realizar modificaciones en su software y en sus funcionalidades, además de facilitar plena movilidad.

Hay que hacer hincapié en que los mayores han valorado favorablemente los dispositivos y funcionalidades TIC como herramienta para mejorar su calidad de vida, pese a un prejuicio inicial que hacía pensar lo contrario. Dadas las escasas, o nulas, alternativas, los mayores del medio rural comienzan a ver las soluciones TIC como una verdadera opción. Por otra parte, estos dispositivos están cada vez más introducidos en nuestra sociedad y los mayores, aunque no los hayan utilizado, sí están más familiarizados con ellos y no descartan convertirse en usuarios. También, gracias a las sesiones de trabajo, los mayores han tenido la posibilidad de tocar, manejar y probar, algunos dispositivos, lo que les ha mejorado la imagen que tenían, ya que su uso no les ha resultado tan difícil.

Esta extraordinaria acogida de las funcionalidades TIC por parte de las personas mayores representa una interesante perspectiva en la investigación. De modo que este trabajo pretende servir de referencia a futuros investigadores, facilitándoles una visión global sobre las preferencias manifestadas por los mayores tanto en lo que se refiere a dispositivos móviles como a funcionalidades, que se convierte, así, en punto de partida para orientar nuevas líneas de investigación y futuros desarrollos tecnológicos en este campo.

Así mismo, también se busca llamar la atención de poderes públicos y compañías privadas para garantizar el servicio en el medio rural, esto es, que el medio rural tenga buena cobertura de telefonía móvil y datos Internet, de modo que se pueda asegurar el correcto funcionamiento de dispositivos e implementación de funcionalidades TIC. Del mismo modo, y tal y como concluye el informe de Naciones Unidas (2015), los gobiernos deberían ayudar a superar la brecha digital al abordar las diferencias en la educación y en las habilidades de las personas mayores, a través de cursos de capacitación tecnológica, programas y centros de aprendizaje adaptados a sus necesidades (United Nations, 2015). 


\section{Agradecimientos}

Esta investigación no hubiera sido posible sin la ayuda de las asociaciones que, amablemente, ayudaron convocando personas mayores con quienes se pudieron hacer los talleres de valoración de los dispositivos móviles. Desde aquí, un sentido agradecimiento a: Asociación de Jubilados y Pensionistas del Bajo Aragón, Asociación de Amas de Casa y Consumidores, Asociación de Jubilados, Asociación de Amas de Casa Inmaculada, Asociación de Amas de Casa Virgen del Carmen, Asociación de Mujeres, Asociación ALFAR, Asociación de Viudas Santa Emerenciana, Asociación de Amas de Casa y Consumidores Santa María, Asociación de Amas de Casa Santa Bárbara, Asociación de Amas de Casa Fuensanta.

\section{Notas}

${ }^{1}$ Es necesario señalar que, a efectos de esta investigación, se considera persona mayor a quien supera los 65 años. Y es que no existe una edad comúnmente aceptada para determinar cuándo una persona entra en la etapa de vejez y dependencia, pues esto atiende tanto a factores colectivos como individuales, pero en este trabajo se hace referencia a la consideración de 65 años, hecha por Naciones Unidas para las personas que viven en países desarrollados (United Nations, 2009). Así mismo, también esta es la edad que hasta ahora se ha considerado como inicio de jubilación en España; por otro lado, es cuando, en algunos casos, comienzan a apreciarse los primeros deterioros propios del envejecimiento; y, en definitiva, es la edad que se considera, de forma más generalizada, como puerta de entrada al colectivo de la Tercera Edad.

${ }^{2}$ Por un lado, se debe advertir que las tecnologías de la información y la comunicación son un campo de reciente desarrollo y nuestra población objeto de estudio es un colectivo de personas mayores de 65 años, de modo que la relación previa ha sido muy reducida o prácticamente inexistente. Hasta este momento las TIC no han tenido un papel destacado en las actividades cotidianas de las personas mayores, como sí se advierte en otros colectivos. Por otro lado, la presencia de las TIC en el medio rural ha sido, si cabe, más reducida, debido a que las actividades laborales (en un inicio, introductoras en el uso de las TIC) que generalmente se desarrollan en él no exigen su utilización, y debido también a que las infraestructuras que requieren no siempre existen en el medio rural o no garantizan un servicio de calidad, como se ha podido observar en la realización de los talleres de participación.

\section{Referencias}

Abascal, J., \& Civit, A. (2001). Mobile Communication for Older People: New Opportunities for Autonomous Life. Workshop on Universal Accessibility of Ubiquitous Computing: Providing for the Elderly. Alcácer do Sal, Portugal. 
Atzori, L., Lera A., \& Morabito, G. (2010). The Internet of Things: A survey. Computer Networks, 54, 2787-2805. doi: 10.1016/j.comnet.2010.05.010

Barnard, Y., Bradley, M.D., Hodgson, F., \& Lloyd, A.D. (2013). Learning to use new technologies by older adults: Perceived difficulties, experimentation behaviour and usability. Computers in Human Behavior, 29(4), 1715-1724. doi: 10.1016/j.chb.2013.02.006

Bareño, R. (2011). Mobile Márketing Máster Class. ITAE, Escuela de Negocios de Extremadura. Recuperado de https://studylib.es/doc/7324019/mobile-m\%C3\%A1rketing-

m\%C3\%A1ster-class-temario

Basterretche, J.F. (2007). Dispositivos móviles. Universidad Nacional del Nordeste. Facultad de Ciencias Exactas, Naturales y Agrimensura. Licenciatura en Sistemas Corrientes - Argentina.

Bell, G. (2006). No More SMS from Jesus: Ubicomp, Religion and Technospiritual Practices. In P. Dourish \& A. Friday (Eds.), UbiComp 2006: Ubiquitous Computing. UbiComp 2006. Lecture Notes in Computer Science, vol 4206 (pgs. 141-158). Berlin: Springer. doi: 10.1007/11853565_9

Bertera, E.M., Tran, B.Q., Wuertz, E.M., \& Bonner, A. (2007). A study of the receptivity to telecare technology in a community-based elderly minority population. Journal of Telemedicine and Telecare, 13(7), 327-332. doi: 10.1258/135763307782215325

Boulos, M.N., Rocha, A., Martins, A., Vicente, M.E., Bolz, A., Feld, R., Tchoudovski, I., Braecklein, M., Nelson, J., Laighin, G.O., Sdogati, C., Cesaroni, F., Antomarini, M., Jobes, A., \& Kinirons, M. (2007). Caalyx: a new generation of location-based services in healthcare. International Journal of Health Geographics, 9(6). doi: 10.1186/1476-072X-6-9

Boz, H., \& Aksoy, M.E. (2011). The internet usage profiles of adults. Social and Behavioral Sciences, 28, 596-600. doi: 10.1016/j.sbspro.2011.11.113

Chau, P.Y.K. (1996). An empirical assessment of a modified technology acceptance model. Journal of Management Information Systems, 13(2), 185-204. doi: 10.1080/07421222.1996.11518128 
Cobo, J.C. (2009). The Information Technologies Concept, Benchmarking of ICT Definitions in the Knowledge Society (El concepto de tecnologías de la información. Benchmarking sobre las definiciones de las TIC en la sociedad del conocimiento). Zer-Revista de Estudios de Comunicación, 14(27), 295-318.

Comeau, D. (2005). Gerontechnology: A Class on Aging with Technology. Center for Studies in Aging. Department of Applied Gerontology, University of North Texas, School of Community Service.

Conci, M. (2007). Mobile Phone Acceptance by Older People. Building and Validation of a theoretical model. Thesis. Universita Degli Studi di Trento.

Davy, A. (2003). Components of a smart device and smart device interactions. MZones, White Paper.

Demiris, G., Rantz, M.J., Aud, M.A., Marek, K.D., Tyrer. H.W., Skubic, M. \& Hussam, A.A. (2004). Older adults' attitudes towards and perceptions of 'smart home' technologies: a pilot study. Medical Informatics \& The Internet in Medicine, 29, 87-94. doi: 10.1080/14639230410001684387

Eisma, R., Dickinson, A., Goodman, J., Syme, A., Tiwari, L., \& Newell, A.F. (2004). Early user involvement in the development of information technology-related products for older people. Universal Access in the Information Society, 3, 131-140. doi: 10.1007/s10209004-0092-z

Enwald, H., Kangas, M., Keränen, N., Korpelainen, R., Huvila, I., \& Jämsä, T. (2016). Opinions and use of mobile information technology among older people in northern finland-preliminary results of a population based study. Proceedings of the Association for Information Science and Technology, 53(1), 1-5.

Fan, Q. (2016). Utilizing ICT to prevent loneliness and social isolation of the elderly. A literature review/Utilizando las TIC para prevenir la soledad y el aislamiento social de las personas mayores. Una revisión literaria. Cuadernos de Trabajo Social, 29(2), 185.

Fernández, A. (2011). Las personas mayores ante las Tecnologías de Información y Comunicación. 60 y más, 300, 8-13. 
Fernández-Caballero, A., González, P., Navarro, E., \& Cook, D. (2017). Pervasive Computing for Gerontechnology. Special Issue. Pervasive and Mobile Computing, 34, 1-2. doi: 10.1016/j.pmcj.2016.12.004

Fundación Telefónica (2016). La sociedad de la información en España, 2016. Madrid: Ariel.

Gaßner, K., \& Conrad, M. (2010). ICT enabled independent living for elderly. A status-quo analysis on products and the research landscape in the field of Ambient Assisted Living (AAL) in EU-27. Institute for Innovation and Technology.

García, B. (Coord.) (1997). Envejecimiento en el mundo rural: problemas y soluciones. Madrid: Ministerio de Trabajo y Asuntos Sociales. IMSERSO.

García, N., \& Bermejo, A.B. (2004). Tecnologías de la información y las comunicaciones para las personas mayores. CEDITEC, Universidad Politécnica de Madrid.

Harrefors, C., Axelsson, K., \& Savenstedt, S. (2010). Using assistive technology services at differing levels of care: healthy older couples' perceptions. Journal of Advanced Nursing, 66(7), 1523-1532. doi: 10.1111/j.1365-2648.2010.05335.x

Harrington, T.L., \& Harrington, M.K. (2000). Gerontechnology: Why and How. Herman Bouna Foundation for Gerontechnology. The Netherlands: Shaker Publishing B.V.

Hill, R., Betts, L. R., \& Gardner, S. E. (2015). Older adults' experiences and perceptions of digital technology: (Dis) empowerment, wellbeing, and inclusion. Computers in Human Behavior, 48, 415-423. doi: 10.1016/j.chb.2015.01.062

Hill, R., Beynon-Davies, P., \& Williams, M.D. (2008). Older people and internet Engagement Acknowledging social moderators of internet adoption, access and use. Information Technology \& People, 21(3), 244-266. doi: 10.1108/09593840810896019

Ijsselsteijn, W., Nap, H.H., Kort, Y., \& Poels, K. (2007). Digital game design for elderly users. Proceedings of the 2007. Conference on Future Play, NY, USA, 17-22.

IMSERSO (2015). Informe 2014. Las personas mayores en España. Colección Documentos. Serie Documentos Estadísticos. 
Karavidas, M., Lim, N.K., \& Katsikas, S.L. (2005). The effects of computers on older adult users. Computers in Human Behavior, 21, 697-711. doi: 10.1016/j.chb.2004.03.012

Kurniawan, S. (2007). Mobile Phone Design for Older Persons. Journal Interactions. July + August, ACM 1072-5220/07/0700. 24-25.

Kurniawan, S. (2008). Older people and mobile phones: A multi-method investigation. International Journal of Human-Computer Studies, 66, 889-901. doi: 10.1016/j.ijhcs.2008.03.002

Ling, R. (2008). Exclusion or self-isolation? Texting and the elderly users.

The information society, 24(5), 1-9. doi:

10.1080/01972240802356125

Mitzner, T.L., Boron, J.B., Fausset, C.B., Adams, A.E., Charness, N., Czaja, S.J., Dijkstra, K., Fisk, A.D., Rogers, W.A., \& Sharit, J. (2010). Older adults talk technology: Technology usage and attitudes. Computers in Human Behavior, 26(6), 1710-1721. doi: 10.1016/j.chb.2010.06.020

Megalingam, R.K., Pocklassery, G, Jayakrishnan, V, Mourya, G., \& Thulasi, A.A. (2014). Smartphone based continuous monitoring system for home-bound elders and patients. International Conference on Communication and Signal Processing, IEEE. April 3-5, India

Melander, A. (2008). Ageing well. Mobile ICT as a tool for empowerment of elderly people in home health care and rehabilitation. Doctoral Thesis. Lulea Univertisy of Technology.

Morillo, J.D. (2011). Introducción a los dispositivos móviles. Barcelona: Universitat Oberta de Catalunya.

Muntaner, A., Vidal-Conti, J., Borràs, P.A., Cantallops, J., Ponseti, F.J., \& Palou, P. (2015). Uso de WhatsApp para la disminución de factores de riesgo cardiovascular en personas mayores. Revista Andaluza de Medicina del Deporte, 8(1), 20-47. doi: 10.1016/j.ramd.2014.10.042

Oksman, V. (2006). Young People and Seniors in Finnish Mobile Information Society. Journal of Interactive Media in Education, 2, 121. doi: $10.5334 / 2006-3$

ONCE (Fundación) \& GRUPO FUNDOSA (2011). Libro Blanco para el diseño de tecnología móvil accesible y fácil de usar. Plan Avanza2. Ministerio de Industria, Turismo y Comercio. (TSI-040200-2009 46. 
Estudio experimental para la identificación y comparación de tecnologías de Internet móvil accesibles y fáciles de usar).

Plaza, I., Martín, L., \& Medrano, C. (2011). Mobile applications in an aging society: Status and trends. The Journal of Systems \& Software, 84, 1977-1988. doi: 10.1016/j.jss.2011.05.035

Pérez, M.P. (2012). Tecnologías móviles y tercera edad. Proyecto fin de carrera. Ingeniería Técnica en Informática de Gestión. Escuela Universitaria Politécnica de Teruel.

Rouse, M. (2006). Mobile Internet device (MID). Retrieved from http://searchmobilecomputing.techtarget.com/definition/mobileInternet-device

Rubio, M.A., Plaza, I., Igual, R., \& Martín, L. (2013). Estudio y selección de tecnologías móviles para los mayores del medio rural turolense. Revista Teruel, 94(II). Instituto de Estudios Turolenses, 137-163.

Rubio, M.A., Plaza, I., \& Orive, V. (2016). Funcionalidades TIC dirigidas a personas mayores. ¿Cómo son valoradas en el medio rural español? Ar@cne, 215.

Sánchez-Valle, M., Abad, M. V., \& Llorente-Barroso, C. (2017). Empowering the Elderly and Promoting Active Ageing Through the Internet: The Benefit of e-inclusion Programmes. In Safe at Home with Assistive Technology (95-108). Springer International Publishing.

Scanaill, C.N., Ahearne, B., \& Lyons, G.M. (2006). Long-term telemonitoring of mobility trends of elderly people using SMS messaging. IEEE Transactions on Information Technology in Biomedicine, 10(2), 412-413.

Selwyn, N., Gorard, S., \& Furlong, J. (2003). The information aged: Older adults' use of information and communications technology in everyday life. Working Paper Series, Paper 36, School of social sciences.

Sum, S., Mathews, R.M., Hughes, I., \& Campbell, A. (2008). Internet use and loneliness in older adults. Cyberpsychology \& Behavior, 11(2), 208-211. doi: 10.1089/cpb.2007.0010

Tamburini, E., \& Paggetti, C. (2005). Remote Management of Integrated Home Care Services. EHTEL Conference, Roma. 
Thayer, S.E., \& Ray, S. (2006). Online communication preferences across age, gender, and duration of internet use. Cyberpsychology \& Behavior, 9(4), 432-440. doi: 10.1089/cpb.2006.9.432

Tangtisanon, P. (2016). Healthcare system for elders with automatic drug label detection (Conference Paper). International Conference on Control, Automation and Systems.

Tennant, B., Stellefson, M., Dodd, V., Chaney, B., Chaney, D., Paige, S., \& Alber, J. (2015). eHealth literacy and Web 2.0 health information seeking behaviors among baby boomers and older adults. Journal of medical Internet research, 17(3), e70. doi: 10.2196/jmir.3992

Triadó, M.C. (2003). Envejecer en entornos rurales. Madrid. IMSERSO. Estudios $I+D+I, \mathrm{n}^{\circ} 19$.

United Nations (2009). World Population Ageing 2009. Department of Economic and Social Affairs. ESA/P/WP/212. December 2009.

United Nations (2015). World Population Ageing Report 2015. Department of Economic and Social Affairs Population Division. United Nations. New York.

Wagner, N., Hassanein, K. and Head, M. (2010). Computer use by older adults: A multi-disciplinary review. Computers in Human Behavior, 26(5), 870-882. doi: 10.1016/j.chb.2010.03.029

Walker, A. (2004). Quality of life in old age in Europe. Growing Older in Europe. Open University Press, Mac Graw Hill Education.

Wang, L., Rau, P.P., \& Salvendy, G. (2011). Older adults' acceptance of information technology. Educational Gerontology, 37, 1081-1099. doi: 10.1080/03601277.2010.500588

Wiles, J.L., Leibing, A., Guberman, N., Reeve, J., \& Allen, R.E. (2012). The Meaning of "Aging in Place" to Older People. The Gerontologist, 52(3), 357-366. doi: 10.1093/geront/gnr098

Wilkowska, W., \& Ziefle, M. (2009). Which Factors Form Older Adults' Acceptance of Mobile Information and Communication Technologies? HCI and Usability for E-Inclusion, Proceedings, 5889, 81-101. doi: 10.1007/978-3-642-10308-7_6

Xavier, F.M., Ferraz, M.P., Marc, N, Escosteguy, N.U., \& Moriguchia, E.H. (2003). Elderly people's definition of quality of life. Revista 
Brasileira Psiquiatria, 25(1), 31-39. doi: 10.1016/02779536(95)00117-P

Xie, B. (2008). Multimodal computer-mediated communication and social support among older Chinese internet users. Journal of ComputerMediated Communication, 13, 728-750. doi: 10.1111/j.10836101.2008.00417.x

María Ángeles Rubio Pastor es profesora del Area de Economía y Gestión del Centro Universitario de la Defensa, Universidad de Zaragoza, España

Inmaculada Plaza García es profesora del Departamento de Ingeniería Electrónica y Comunicaciones de la Escuela Universitaria Politécnica de Teruel, Universidad de Zaragoza, España

Víctor Orive Serrano es profesor de la Escuela Universitaria de Turismo de la Universidad de Zaragoza, España

Dirección de contacto: Centro Universitario de la Defensa, Universidad de Zaragoza. Ctra. de Huesca, s/n -50090- Zaragoza, España.Email: marubio@unizar.es 


\section{RIMCIS \\ Social Sciences}

\section{Hipatia Press}

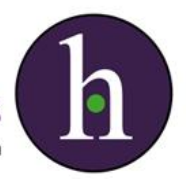

Instructions for authors, subscriptions and further details:

http://rimcis.hipatiapress.com

\section{The Growth of E-Marketing in Business-to-Business Industry and its Effect on the Performance of Businesses in Pakistan: Marketing Success}

Adnan Ahmed Sheikh ${ }^{1}$, Arfan Shahzad ${ }^{1}$, Awanis Ku Ishak ${ }^{1}$

1) Universiti Utara Malaysia

Date of publication: July $30^{\text {th }}, 2017$

Edition period: July 2017 - November 2017

To cite this article: Sheikh, A.A., Shahzad, A., \& Ku Ishak, A. (2017). The Growth of E-Marketing in Business-to-Business Industry and its Effect on the Perfromance of Businesses in Pakistan: Marketing Success. International and Multidisciplinary Journal of Social Sciences, 6(2), 178-214. doi: 10.17583/rimcis.2017.2704

To link this article: $\underline{\text { http://doi.org/10.17583/rimcis.2017.2704 }}$

\section{PLEASE SCROLL DOWN FOR ARTICLE}

The terms and conditions of use are related to the Open Journal System and to Creative Commons Attribution License (CC-BY). 


\section{The Growth of E-Marketing in Business-to-Business Industry and its Effect on the} Performance of Businesses in
Pakistan: Marketing Success

Adnan Ahmed Sheikh

Universiti Utara Malaysia

Awanis Ku Ishak

Universiti Utara Malaysia
Arfan Shahzad

Universiti Utara Malaysia

\section{Abstract}

E-marketing technology usage continues to vary, even with widespread acceptance of the Internet by several organizations. However, there is a lack of empirical studies regarding the use of e-marketing in the small and large businesses of Pakistan and their resulting performance effects. Therefore, the purpose of this paper is to examine the technological, organizational and environmental factors on the business performance working in Pakistan by including use of e-marketing as a mediating variable. Data has collected from marketing department of 259 businesses located in several cities. Results revealed from PLS-SEM showed eight out of nine significant hypothesis directly with use of e-marketing and firm performance and also by including use of e-marketing as a mediating variable, all results found significant except market orientation. Moreover, the results of this study have key implications for the marketing field, as they strain the vital role of marketing persons in the effective implementation of e-marketing in the firms.

Keywords: e-Marketing uses, technology, firm performance, manufacturing, small and large businesses 


\section{El Crecimiento del}

\section{E-Marketing en la Business-to-}

Business Industry y su Efecto en el Rendimiento de las Empresas en Pakistán: Éxito del Marketing

Adnan Ahmed Sheikh

Universiti Utara Malaysia

Awanis Ku Ishak

Universiti Utara Malaysia
Arfan Shahzad

Universiti Utara Malaysia

\section{Resumen}

El uso de la tecnología e-Marketing sigue variando, a pesar de la extendida aceptación de Internet por parte de varias organizaciones. Sin embargo, hay una falta de estudios empíricos sobre el uso del e-marketing en las pequeñas y grandes empresas en Pakistán y sobre su rendimiento. Por eso, el objetivo de este artículo es examinar los factores tecnológicos, organizacionales y ambientales sobre el rendimiento de las empresas en Pakistán que han incluído el uso del e-marketing como una variable interviniente. Se han recogido datos de los departamentos de marketing de 259 empresas localizadas en varias ciudades. Los resultados del PLSSEM muestran que ocho de nueve hipótesis significativas están relacionadas directamente con el uso del e-marketing y con el rendimiento de la empresa, también al incluir el uso del e-marketing como una variable interviniente, todos los resultados salieron significativos salvo la orientación de mercado. Aún más, los resultados de este estudio tiene implicaciones importantes para el campo del marketing, ya que atrae el rol vital de técnicos en marketing hacia la implementación efectiva del e-marketing en las empresas.

Palabras clave: usos del e-marketing, tecnología, rendimiento empresarial, manufactura, pequeña y grande empresa

2017 Hipatia Press

ISSN: 2014-3680

DOI: $10.17583 /$ rimcis.2017.2704 
$\mathbf{R}$ ecently, the fact that technological change and innovation are fundamental sources of productivity and sustainable growth for a business is widely accepted (Arifin \& Frmanzah, 2015; Johnson, Scholes \& Fréry, 2002; Markides, 1997; O’Mahony \& Ark, 2003). Accordingly, technology adoption and usage is a strategic innovation that is a novel way of engaging with competitors and of improving the financial performance of existing businesses (Ireland \& Webb, 2007; Riddell \& Song, 2012). Therefore, the successful adoption of technology in the firm significantly "affects industrial competitors" toward achieving growth in the firm's performance (Arifin \& Frmanzah, 2015; Barney, 1991; Cornford \& Smithson, 2006; Das, Majumdar, Chakrabarti \& Chakrabarti, 2013; Kotler \& Keller, 2006; Porter, 1985, 1990; Rayport \& Jaworski, 2004).

Several studies have discussed the use of modern technology that will enhance firm performance in terms of firm productivity, financial performance, market share growth, and customer loyalty (Lopez-Acevedo, 2002; Lucia-Palacios, Bordonaba-Juste, Polo-Redondo \& Grünhagen, 2013; Mutlu \& Sürer, 2015). Many researchers argued that technology adoption and usage lowers the overall operational costs (Benitez-Amado, LlorensMontes \& Perez-Arostegui, 2010) by improving efficiency and increasing effectiveness (Rusli, 2012; Sabbaghi \& Vaidyanathan, 2008). Furthermore, the industrial sector performance in any country is considered its economic engine, which continuously contributes to worldwide economic development.

Currently, industrial sector performance has gained considerable attention from many academicians and practitioners (Arifin \& Frmanzah, 2015). Substantial interest in the field of innovation has significantly supported business operations and aided firms in enhancing their global reach. However, the recent revolution in e-marketing has notably affected the performance of businesses (Eid \& El Gohary, 2013; El Gohary, 2012). Therefore, a growing number of organizations are currently using the Internet or other electronic tools to communicate with their trading partners, government institutes, customers, and end-users of their products and services (Ahmad, Rahim, Bakar \& Mohamed, 2014; El Gohary, 2010, 2012). 
Some studies and findings have also corroborated that a technological communication gap remains extant between the firms and their customers. Likewise, negative market trends, growing global competition, and staff with insufficient ICT skills are other factors for the firms' non-adopting behavior regarding the e-Business model, which significantly affects the relative advantage of electronic business (Morais, 2006; Tandon \& Reddy, 2013). However, e-commerce in organizations is growing rapidly to cater to maximum global market shares; for example, a $120 \%$ increase in ecommerce trade has been reported in China (Fredriksson, 2013). Conversely, businesses in Pakistan remain reluctant to properly implement e-marketing despite being a cost-effective way to conduct business in this global era.

For this current research, the definition by Straus and Frost (2001) has been used to define e-marketing as follows: "The use of electronic data and applications for planning and executing the conception, distribution and pricing of ideas, goods and services to create exchanges that satisfy individual and organizational objectives". The dawn of the 21 st century corresponds to the revolution of novel ideas that are radically influencing the paradigms in almost every field of human activity. However, the world has experienced the demise of severe geographical and communication segregation. In addition, as an economic event driven by technology, globalization has manifested in a shift from a world of diverse national economies to a global economy. Globalization has become a reality and a key economic factor nowadays (Noshab, 2006; Sheikh, Shahzad \& Ishak, 2016; Ueasangkomsate, 2015).

In particular, Pakistan has very few businesses using e-business for online sales and promotion especially in $\mathrm{B} 2 \mathrm{C}$ markets. Internet users in Pakistan rose to 10.3 million after the launch of $3 \mathrm{G}$ and LTE. The scenario that $28 \%$ of the country would have Internet access in the next five years is anticipated. However, with numerous new online projects emerging rapidly and extant businesses expecting good growth rates and improved performances, an extensive gap exists in terms of maximizing the ecommerce potential and competing with major regional players (Junaid, n.d.). Mącik, Jóźwik, and Nalewajek (2012) validated that firms are changing their structure to become globalized. According to the World Trade Organization report issued in 2016 regarding the textile growth trend, 
Pakistan faces stagnation and even downfall in the overall textile growth, whereas China, India, and Bangladesh have maintained their positions, with India and China in the top three rankings.

Studies have reported mixed findings on the direct effects of e-marketing usage on firm performance. However, few evidence supports the positive impact of e-marketing on firm performance, such as firm growth (Mohamad, Building \& Ismail, 2009; Raymond, Bergeron \& Blili, 2005), financial gain (Johnston, Wade \& McClean, 2007), and competitive advantage (Teo, 2007; Teo \& Pian, 2003). The benefits achieved were inconsistent in different sectors given the sizes and regions (Johnston \& Wright, 2004). However, in the same perspective, the realized benefits were positively associated with the use of e-marketing (Raymond et al., 2005; Sam \& Leng, 2006). Despite all the arguments, the use of e-marketing enhances firm performance. Moreover, studies that linked these two variables are limited. Previous studies have commonly used e-business, e-commerce, and innovation as mediators of firm performance. However, e-marketing as a mediator of firm performance is still neglected by previous researchers.

Moreover, scholars have traditionally focused on western organizations. E-marketing practices increase performance and are necessary for Pakistani organizations, particularly in the textile sector (Lucia-Palacios et al., 2013; Voola, Casimir, Carlson \& Anushree Agnihotri, 2012). Furthermore, Iddris and Ibrahim (2015) recommended that establishing a relationship between the use of e-marketing factors and firm performance is necessary. Therefore, the current study examines the effects of e-marketing usage on the performance of small and large businesses in Pakistan. Moreover, Pakistan has lagged in terms of Internet growth. Additionally, its textile sector has been outperformed by regional competitors. In the last few years, access to a global market has notably increased with the help of the Internet. Therefore, organizations operating in these countries are approaching foreign buyers, communicating with their trading partners, and developing ties with government departments to enhance their financial strength and market growth and to satisfy their customers by providing e-marketing services.

Literature in electronic marketing and performance of firms in developing economies is noticeably limited, and the study is extended to include the theories of the adoption and usage of new technology and further 
the impact of such technology for the adoption and use of e-marketing in industries. Further, with new advancements and technologies, some privacy and security issues emerged aside from cultural difficulties, technological barriers, lack of top management support, lack of government support and trading partners, limited opportunities to negotiate on prices, and variation in languages (El Gohary, Trueman \& Fukukawa, 2008; El Gohary, 2010). This finding is in accord with El Gohary (2012), who revealed that the emarketing technology is still considered a novel idea, specifically for businesses working in developing countries that have limited funds and tough competition. Accordingly, businesses cannot afford risky investments or irrational decisions, which caused inadequate knowledge of electronic marketing technology among organizations.

\section{Literature Review and Hypothese Development}

\section{Technological Context and Firm Performance}

In the study by Ahmad, Rahim, Bakar and Mohamed (2014) and a few other studies have highlighted that, relative advantage positively affects the firm's performance, such as growth, financial gain and competitive advantage (Johnston et al., 2007; Qureshi, Keen \& Kamal, 2010; Raymond et al., 2005; Teo, 2007). However, the current study is focused to investigate that, how the textile firms perceives those benefits which are linked with use of emarketing such as; increase of profits \& revenues, cost reduction, customer service quality, business operations stability and lastly, the development of new local and foreign market segments.

Furthermore, most of the studies have conducted a research on relative advantage in the context of the SME's and service sector of western countries and the few developing countries as well (Grandon \& Pearson, 2004; Li, 2008; Molla \& Licker, 2005; Rahayu \& Day, 2015; Rahim et al., 2015). But limited studies has been done in the context of Pakistan and particularly the textile sector has been ignored by the researchers as it is a major sector of Pakistan as compare to other manufacturing sectors. So, to clarify the importance of relative advantage towards firm performance, an 
empirical study is needed. Hence, based on the above argumentation, the recommended hypothesis of this research is:

H1: Relative advantage significant positively related to the firm performance.

\section{Organizational Context and Firm Performance}

The extent to which CEOs impact the firm performance is considerably important to scholarly understanding of, how organizations operate; until now, this relation is poorly implicit. Previous empirical studies examined the relationship among CEOs and firm performance used adjustments, though challenging, however suffer from methodological problems, which systematically reduces the relative influence of CEOs on the performance of the firm as contrast to industry and firm effects. However, in this current study, it needs to examine that, how much variance in firm performance explained by heterogeneity in CEOs of small and large businesses in Pakistan (Mackey, 2008; Rahim et al., 2015; Varukolu, 2007).

Furthermore, most of the studies has used top management support as a predictor in the context of the SME's and service sector of western countries as well as in few emerging countries like China, India, Egypt and Taiwan (Al-qirim, 2007; Duan et al., 2012; El Gohary, 2012; Li, 2008; Molla \& Licker, 2005; Prasad et al., 2001; Premkumar \& Roberts, 1999; Wang et al., 2010). But, on the other hand, limited studies have been done in the context of Pakistan and particularly textile sector has been ignored by the researchers as top management participate in almost all the decisions of the firms, secondly, textile sector in Pakistan is consists of both large and SME's which are mainly owned by the individuals or by families. Thus, in order to clarify the importance of top management support towards firm performance, further empirical investigation is a major focus for current study and needs to fill this gap (PACRA, 2011; Seyal et al., 2004).

H2: Top management support significant positively related to the firm performance. 
With regard to market orientation, market oriented firms grasp the cultural features that allows them to examine their current capabilities, antedate future capabilities and re-design processes in order to support new ones. Although, there is an evidence, for instance (Deshpande, Farley \& Webster, 1993; Narver \& Slater, 1990) of the relationship between MO and business performance. Therefore, MO can be a basis of competitive advantage only when, if it is rare in a company industry (Barney, 1991). With the widespread research on market orientation and its relationship with firm performance, firms are increasingly investing in market orientation because it is a capability that involves information generation within firms through external environment. MO is likely to be a rare, valuable and inimitable capability and should consequently generate competitive advantage and improve firm performance as it involves processes and skills that make it difficult for competitors to copy as suggested by RBV theory (AtuaheneGima \& Murray, 2004). Hence, based on above discussion, the suggested hypothesis is;

H3: Market orientation significant positively related to the firm performance

\section{Environmental Factors and Firm Performance}

A study by Shaher (2012) found that small and large businesses are mainly affected by the ineffective governmental policies, such as lack of R\&D and IT implementation. This implies that, IT needs vital attention and development in small and large businesses of Pakistan to avail the opportunities and get maximum market share with respect to its competitors. However, the gap in previous studies was that, they use government support as a predictor in the studies related to technology adoption (e.g. related to innovation, e-business or e-commerce, e-marketing, e-procurement, egovernment) based on (Kazungu \& Panga, 2015; Tam, 1998; Thatcher et al., 2006) . But very limited studies, that have investigated the impact of government support on firm performance (Shah, Warraich \& Kabeer, 2012), so this is one of the issue which needs to be addressed with the role of government support on textile sector performance in the context of Pakistan. However, the results add a positive contribution to the current knowledge of 
the study with the help of DOI, RBV theory and TOE framework. Hence, based on the past studies, the suggested hypothesis is;

H4: Government support significant positively related to the firm performance.

\section{Use of E-marketing as a Mediator}

\section{Use of E-Marketing and firm Performance}

A study conducted by Mzee, Ogweno and Irene (2015) reveals that, organizations who use the E-Marketing in their businesses found that, implementation of E-Marketing is not an expenditure. It will help to communicate with the customers locally and globally. In line, the study of Rahim et al. (2015) highlights that, e-marketing technology uses differs from business-to-business, in spite of extensive acceptance of the internet in business environments. Other than that, e-marketing is a recent technology that has established considerable attention in the industry. Nevertheless, Chen and Lien (2013) claimed that, in spite of the rapid growth of new technologies, leading organizations still use them at a sluggish rate. The study of El Gohary (2012) specified that, the use of e-marketing technology is relatively a new concept, particularly for organizations working in the developing countries, who have inadequate resources and tough competition. Consequently, businesses might not afford to make un-wise investments or incorrect decisions, which caused poor up-take of e-marketing technology between firms and influence the marketing performance of the firm. Hence, on the basis of previous studies, the suggested hypothesis is;

H5: Use of E-Marketing significant positively related to the firm performance.

\section{Use of E-Marketing relationship with technological factors and firm performance}


In the study by Ahmad, Rahim, Bakar and Mohamed (2014) and a few other studies have highlighted that, relative advantage positively affects the firm's performance, such as growth, financial gain and competitive advantage (Johnston et al., 2007; Qureshi, Keen \& Kamal, 2010; Raymond et al., 2005; Teo, 2007). However, the current study is focused to investigate that, how the textile firms perceives those benefits which are linked with use of emarketing such as; increase of profits \& revenues, cost reduction, customer service quality, business operations stability and lastly, the development of new local and foreign market segments.

Furthermore, most of the studies have conducted a research on relative advantage in the context of the SME's and service sector of western countries and the few developing countries as well (Grandon \& Pearson, 2004; Li, 2008; Molla \& Licker, 2005; Rahayu \& Day, 2015; Rahim et al., 2015). But limited studies has been done in the context of Pakistan and particularly the textile sector has been ignored by the researchers as it is a major sector of Pakistan as compare to other manufacturing sectors. So, to clarify the importance of relative advantage towards firm performance, an empirical study is needed to test the relationship in textile sector of Pakistan to evaluate the findings (Eid \& El Gohary, 2013; Haider et al., 2015; Iddris \& Ibrahim, 2015; Pakistan, 2015). Thus, on the basis of past studies, the suggested hypothesis is;

H6: Relative advantage significant positively related to use of e-marketing.

H7: Use of e-marketing mediates the relationship among relative advantage and performance of firm.

\section{E-Marketing uses association with organizational factors and firm performance}

In a study conducted by Haugh and Robson (2005) found that, those firms in which top management commitment is more towards adoption process are likely to adopt information technology more rapidly. Consistent with this argument, management support has a strong influence on the implementation of infusion and diffusion systems of intranet in the firms. Therefore, a 
researcher claims that, management support motivates to adopt e-marketing technology by giving strength to the firm's technology to become more efficient in internal processes as well as in external communication to reduce customer and trading partner's communication gap and to increase its credibility in the market.

Next, several studies have indicated that, structure of the organizations are becoming more complex and with such density the focus has shifted to include more factors that directly impact on the use of technology within the context of organization. Further examined that, the top management factors could be essential for the success of information systems within organizations because top management support is found as a significant predictor of Internet adoption (Fink, 1998; Premkumar \& Roberts, 1999; Tan \& Teo, 1998; Thong \& Yap, 1995). Thus, based on the past studies, the recommended hypothesis is;

H8: TMS significant positively related to use of e-marleting.

H9: Use of e-marketing mediates the relationship among TMS and performance of firm.

Besides, There is evidence that, firm capabilities influence innovation adoption (Hult, Hurley \& Knight, 2004). There is also evidence that MO positively influences innovation adoption, for instance (Narver et al., 2000), argues that there is a positive relationship between innovation and MO. The current study conceptualizes E-business adoption or E-Marketing adoption/ uses as an innovation. Moreover, Wu et al. (2003) define it as "the use of internet technologies that link customers, suppliers, business partners and employees by adopting at least one of the following activities: (I) websites that offer sales transactions; (ii) customer service websites; (iii) intranets; (iv) extranets; and (v) IP electronic data interchange". Previous studies have shown that, customer orientation positively affects electronic marketing adoption. Furthermore, Han, Kim and Srivastava (1998) define customer orientation as "the adoption of a continuous, pro-active nature toward meeting customer's requirements, which is conceptually similar to MO.Thus, based on the past studies, the suggested hypotheses are; 
H10: Market orientation significant positively related to use of e-marketing. H11: Use of e-marketing mediates the relationship among market orientation and performance of firm.

\section{Use of E-Marketing relationship with environmental factors and firm performance}

Government is reflected as the main factor in the adoption and uses of any innovative technology. Government pushes the firm to adopt and use B2B electronic marketplace in order to operate their marketing-activities and even though governmental intervention in Asian countries has motivated the firms to implement B2B e-business, as it was considered as an important portion of the business environment. Therefore, according to $\mathrm{Hu}, \mathrm{Wu}$ and Wang (2004) a country's willingness for businesses based on electronic media fundamentally depends on the government backing: promotional activities, governmental grants and also the regulatory values for establishing digital trading environment. But, in order to see the government encouragement for e-marketing usage, an empirical study is required.

Moreover, Seyal et al. (2004) clearly highlights that, the influence of government policies and creativities has shown direct as well as indirect motivation to provide information that produces faster technology. For many companies, government has become a source of funding-infrastructure, but to see the role of government in textile sector, there is a need for more investigation. Therefore, on the recommended hypotheses is;

H12: Government support significant positively related to use of emarketing.

H13: Use of e-marketing mediates the relationship among government support and performance of firm. 
Conceptual framework

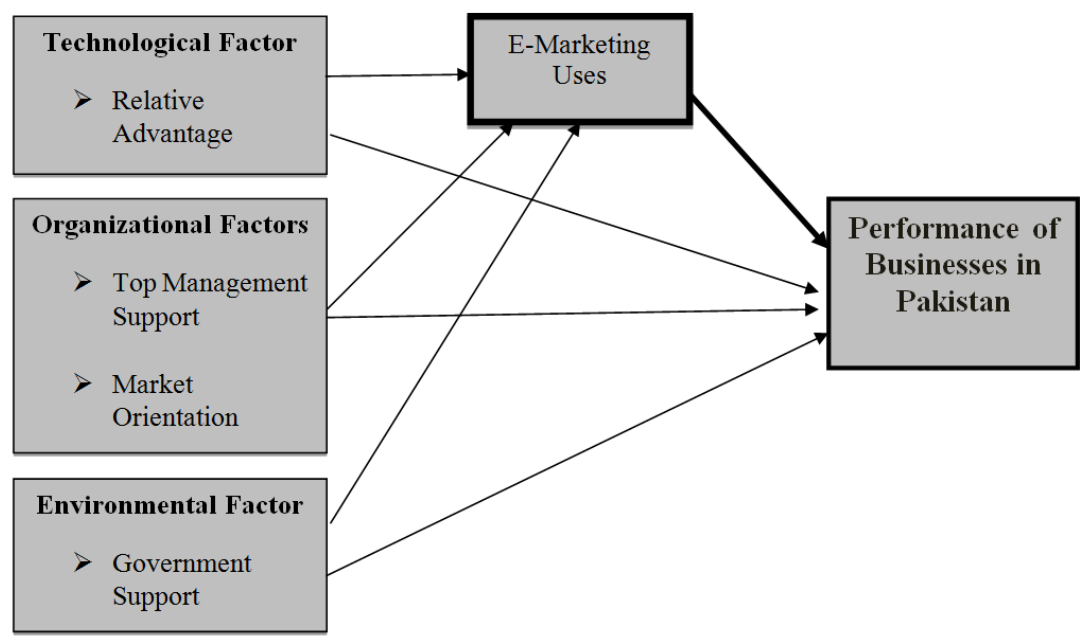

Figure 1. Framework of the Study

\section{Research Methodology}

\section{Data Collection}

The respondents employed for this research are the general manager marketing working in small and large firms of Pakistan. Although, the selected firms are situated in the Punjab and Sindh states of Pakistan as these two states are the main hubs for all type of business. Though, the study is focused on the manufacturing sector due to the main GDP contribution of manufacturing sector in Pakistan. According to Trade Development Authority of Pakistan (TDAP) and Small and Medium Enterprise Development Authority of Pakistan (SMEDA) (2015-2016) report, more than 1500 businesses are working in these two states, which are registered manufacturing firm of Pakistan. Though, according to the rule of thumb by (Hair et al., 2014), only 259 small and large manufacturing firms were selected from Punjab and Sindh states to fulfil the need of this research. However, by using a cluster proportionate sampling technique the firms have 
been selected according to their location and numbers from particular area / cities.

Table 1.

Response from respondents

\begin{tabular}{ll}
\hline Response & Frequency/Rate \\
\hline No of Questionnaires & $\mathbf{4 8 1}$ \\
Questionnaires Received Back & $\mathbf{2 7 8}$ \\
Returned \& Useable Questionnaires & $\mathbf{2 5 9}$ \\
Excluded Questionnaires & $\mathbf{1 9}$ \\
Questionnaires not received from respondents & $\mathbf{2 0 3}$ \\
Total Response Rate & $\mathbf{5 7 . 8 0 \%}$ \\
Valid Response after data entry & $\mathbf{5 3 . 8 5 \%}$ \\
\hline
\end{tabular}

Hence, table 1 shows that only 259 questionnaires were used for subsequent analysis that gives a valid response rate of $54 \%$ and total response of $58 \%$. The response rate was obtained comparable to other several past studies using managers of manufacturing sector for instance, $68 \%$ by (Hassan, Mukhtar, Qureshi \& Sharif, 2012) in the context of Pakistan. According to (Hair, Black, Babin, Anderson \& Tatham, 2010) good sample size for the purpose of statistical or quantitative analysis must be at least 10-20 times higher than the total number of variables. Thus, the sample size of the present study is 220 according to rule of thumb suggested by Hair et al. (2014), however, researcher has received 259 questionnaires from the firms, which appear to be much suitable for statistical analysis.

\section{Determination of the Sample Size}

After making an area cluster, proportionate sampling has been used with multi-stage sampling, at first stage, the researcher selected overall Pakistan country, after that, the researcher segregates the regions according to the four provinces of Pakistan, which are Punjab, Sindh, NWFP and Baluchistan, in a third step, among four provinces, two provinces were listed down which are Sindh and Punjab province. Though, Punjab and Sindh has further divided into six parts or cities, according to the manufacturing mills location mentioned in APTMA, SMEDA and APBUMA list. Moreover, 
these six clusters are Lahore, Multan, Faisalabad, Gujranwala, Rahimyar Khan and Karachi. It has been identified on the basis of location (area) of the small and large manufacturing firms in Pakistan. Specifically, Punjab province has been selected because most of the agro-based firms are operating here. After that, Sindh province includes the sea port in Karachi city which is considered as the hub for imports and exports of the country. However, for the present study, a multi- stages cluster sampling method was used to collect the required number of data for analysis (Allen et al., 2003).

\section{Research Measures}

The variables and items of current research have been adapted from the past literature. Though, the research questionnaire is divided into two major parts. The first part contains seven Likert-type scale items and the second part intended to get the demographic data of the respondents. The Likert scale is intended to notice the degree of response about how sturdily the respondents get agree and disagree with a specific question (Sekaran, 2006). Additionally, the aim to select 7-point Likert scale is to provide additional choices to the respondents to the internment of the better capriciousness of respondents attitude and behavior (Hinkin, 1995).

Therefore, several items have been selected, likewise, 12 item scale to measure market orientation has been adapted as given by Narver and Slater (1990). Moreover, 4 item scale to measure relative advantage and 4 items to measure top management support has been adapted from Premkumar, Ramamurthy and Nilakanta (1994). Besides, 6 item scale to measure government support has been adapted from Molla and Licker (2005). Additional, 8 item scale to measure use of e-marketing has been adapted from (Srinivasan, Lilien \& Rangaswamy, 2002). Finally, 7 item scale to measure firm performance was adapted from Hooley et al. (2005). 


\section{Data Analysis and Results}

\section{Assessment of the Measurement Model}

Before going to assess the measurement model, assumptions of multivariate analysis instructions regarding screening of the missing data and outlier's has been examined. For instance, regarding missing data, it has been revealed that, one missing value and a mean substitution is used to provide the replacement values for missing data. However, both the multivariate outlier's and the uni-variate has found in the data-set and since the variables of the current study was measured on the basis of 7-point likert scale, and even though not a single observation seems to be on extreme. Thererfore, the data considered for current study has been kept for analysis.

Furthermore, the PLS-SEM technique has been used for the theory confirmation and also recommends, where the relationship may exists. However, in evaluating the model, the measurement model has undertook to insure the model reliability as well as validity. Based on PLS (SEM), the assessment of measurement model has been analysed properly by using SmartPLS 3.0 (Ringle et al., 2015). To conclude the measurement model discriminant validity, reliability and convergent validity of the measurement of constructs was observed and keenly examined. The Fig 2 demonstrates the measurement model of current study and on the basis of this, the table 4.1 has been explained.

Before examining the convergent validity, the researcher examined the loadings and cross loading of all the given items of current study variables in order to point out the problems that work as a pre-requirements for the measurement model. According to by Hair et al. (2010, p. 14), the convergent validity is attained when the factor loadings of all the items is higher than the value of 0.5 and none of the loading of any item from other constructs have the higher loadings than the one which suppose to measure. Concerning this current study, loadings are above the threshold of 0.5 as shown Table 2. Moreover, Table 4.1 explains composite reliability, average variance extracted (AVE) and Cronbachs alpha values of all the study constructs. 
Moreover, according to (Fornell \& Larcker, 1981; Hair et al., 2014) the AVE should be atleast 0.50 and the composite reliability must be accepted at least 0.70 and higher. However, as explained in the given Table 2, all the variables have the high reliability and the average-variance-extracted (AVE) is higher than the threshold value of 0.50 which indicates the reliability of measurement model. The current study calculated the Cronbachs-Alpha to find out, "internal consistency" of the data. Furthermore, George and Mallery (2003) provided the rule of deciding the value "alpha; " $\alpha>0.9$ Excellent, $\alpha<0.8$ - Good, $\alpha<0.7$ - Acceptable". However, according to the results of current study as mentioned in Table 4.2 clearly highlights that all variables have "Cronbachs Alpha" value more than 0.6. So this reveals that, all study variables have good consistency.

Moreover, discriminant validity was analysed to determine the model external consistency, on the basis of correlation among the latent variables, however, the value of the variables were compare with the square root of AVE's. Finally, as explained in table 3 all the correlations among the variables are lower than square root-averages (AVEs) as which are bolded in crosswise.

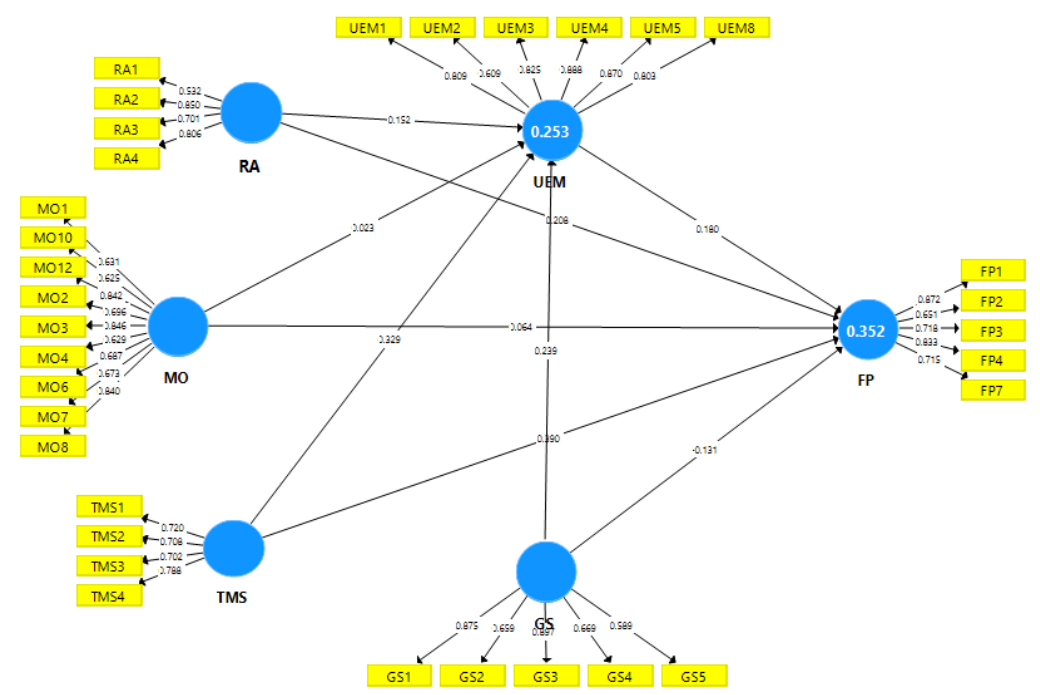

Figure 2. Measurement Model 
Table 2.

Findings of the Measurement Model

\begin{tabular}{|c|c|c|c|c|c|}
\hline Construct (s) & Item (s) & Loading's & $\begin{array}{c}\text { Cronbach's- } \\
\text { Alpha }\end{array}$ & $\begin{array}{l}\text { Composite- } \\
\text { Reliability }\end{array}$ & $\begin{array}{l}\text { Average- } \\
\text { Variance } \\
\text { Extracted }\end{array}$ \\
\hline \multirow{6}{*}{$\begin{array}{l}\text { Government } \\
\text { Support (GS) }\end{array}$} & GS1 & 0.875 & 0.795 & 0.861 & 0.560 \\
\hline & GS2 & 0.659 & & & \\
\hline & GS3 & 0.897 & & & \\
\hline & GS4 & 0.669 & & & \\
\hline & GS5 & 0.589 & & & \\
\hline & FP1 & 0.872 & 0.821 & 0.873 & 0.581 \\
\hline \multirow{4}{*}{$\begin{array}{l}\text { Firm } \\
\text { Performance } \\
\text { (FP) }\end{array}$} & FP2 & 0.651 & & & \\
\hline & FP3 & 0.718 & & & \\
\hline & FP4 & 0.833 & & & \\
\hline & FP7 & 0.715 & & & \\
\hline \multirow{9}{*}{$\begin{array}{l}\text { Market } \\
\text { Orientation } \\
\text { (MO) }\end{array}$} & MO1 & 0.631 & 0.897 & 0.907 & 0.525 \\
\hline & MO10 & 0.625 & & & \\
\hline & MO12 & 0.842 & & & \\
\hline & $\mathrm{MO} 2$ & 0.696 & & & \\
\hline & $\mathrm{MO} 3$ & 0.846 & & & \\
\hline & MO4 & 0.629 & & & \\
\hline & MO6 & 0.687 & & & \\
\hline & MO7 & 0.673 & & & \\
\hline & MO8 & 0.840 & & & \\
\hline \multirow{4}{*}{$\begin{array}{l}\text { Top } \\
\text { Management } \\
\text { Support (TMS) }\end{array}$} & TMS1 & 0.720 & 0.757 & 0.820 & 0.533 \\
\hline & TMS2 & 0.708 & & & \\
\hline & TMS3 & 0.702 & & & \\
\hline & TMS4 & 0.788 & & & \\
\hline \multirow{4}{*}{$\begin{array}{l}\text { Relative } \\
\text { Advantage } \\
\text { (RA) }\end{array}$} & RA1 & 0.532 & 0.692 & 0.814 & 0.530 \\
\hline & RA2 & 0.850 & & & \\
\hline & RA3 & 0.7020 & & & \\
\hline & RA4 & 0.806 & & & \\
\hline \multirow{6}{*}{$\begin{array}{l}\text { Use of } \\
\text { E-Marketing } \\
\text { (UEM) }\end{array}$} & UEM1 & 0.809 & 0.890 & 0.916 & 0.649 \\
\hline & UEM2 & 0.609 & & & \\
\hline & UEM3 & 0.825 & & & \\
\hline & UEM4 & 0.888 & & & \\
\hline & UEM5 & 0.870 & & & \\
\hline & UEM8 & 0.803 & & & \\
\hline
\end{tabular}


196 Sheikh, Shahazad \& Ishak - The Growth of e-Marketing

Table 3 .

Discriminant-validity of the Constructs

\begin{tabular}{lcccccc}
\hline & FP & GS & MO & RA & TMS & UEM \\
\hline FP & $\mathbf{0 . 7 6 2 3}$ & & & & & \\
GS & -0.0030 & $\mathbf{0 . 7 4 8 1}$ & & & & \\
MO & 0.1635 & -0.1737 & $\mathbf{0 . 7 2 4 6}$ & & & \\
RA & 0.3740 & 0.0912 & 0.2063 & $\mathbf{0 . 7 3 2 5}$ & & \\
TMS & 0.5086 & 0.1680 & 0.0693 & 0.2951 & $\mathbf{0 . 7 3 0 3}$ & \\
UEM & 0.3620 & 0.3047 & 0.0353 & 0.2753 & 0.4160 & $\mathbf{0 . 8 0 5 8}$ \\
\hline
\end{tabular}

\section{Structural Model Assessment}

After the assessment of measurement model, the structural model was assessed by using SmartPLS 3.0. However, following tests in the the structural model has took place which includes; "hypothesis testing with path coefficient and T-value, effect size and predictive relevance of the model were examined".

\section{Direct Effect and Hypothesis Testing}

In SmartPLS, structure model gave inner-modeling analysis of the direct association between the variables of the study icluding t-values and path coefficients. As claimed by Henseler et al. (2009), the path coefficients are similar to regression analysis and standardized beta coefficient. Wherever, the beta values are the coefficients of regression and t-values are analysed to decide the significance level of the constructs. However, on the basis of rule of thumb revealed by Hair et al. (2014), which explains that Bootstrapping method was performed (with 5000 sampling iterations for 259 cases / observations) to determine the beta-values of the coefficient of the regression and also the t-values which must be greater than 1.64 in order to considered resulted value significant. So that to use the derived results for further decision mking on the proposed hypothesis.

The main objective of current study is to weight on model evaluation by analysing the direct relationships and also to test the proposed hypothesized 
relationships among the variables with the help of structural model. However, in current study nine (09) hypotheses having direct relationship's were analysed, from which seven (7) out of nine (09) were resulted supported and only two (02) were resulted not supported. Moreover, Fig 3 demonstrates the direct effect of every predictor on the dependent variable.

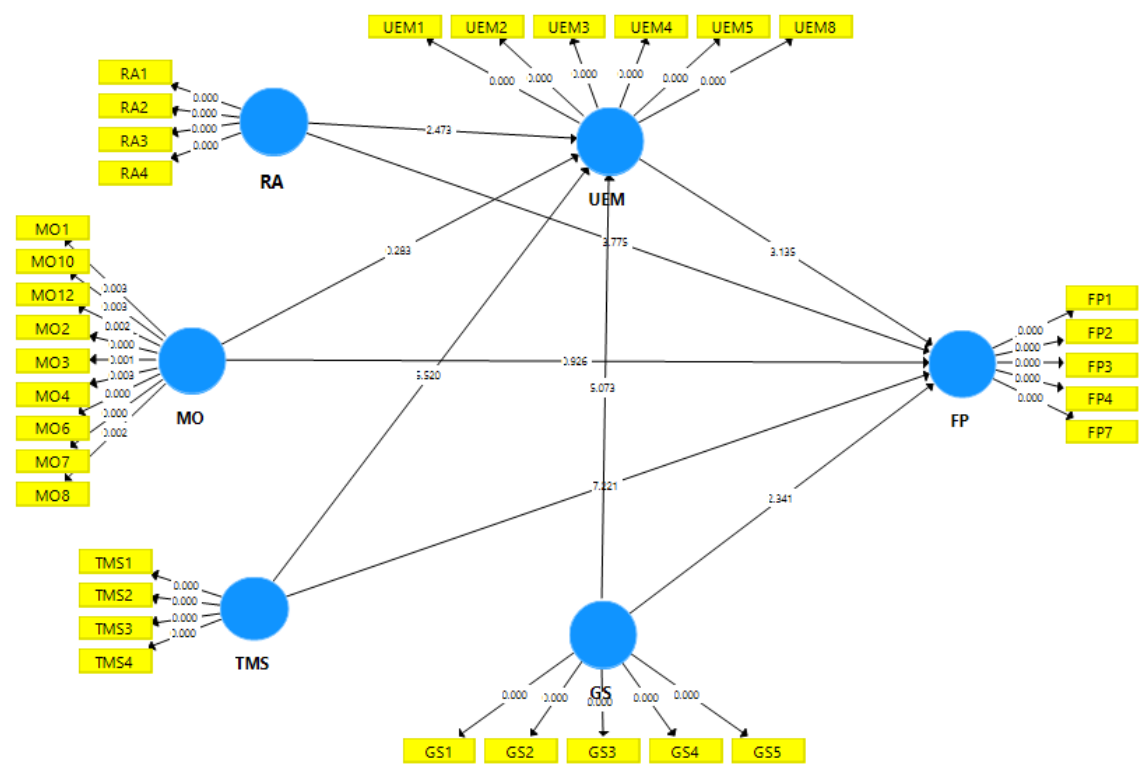

Figure 3. The Structural Model Direct Relationships

Table 4 shows that all hypotheses which has been accepted and supported must have t-value greater than the threshold value of 1.64 and those hypotheses whose t-value (s) are less than 1.64 has been rejected otherwise. Furthermore, Fig. 3 fully explains Table 4.3 and highlights the effect of all variables on the dependent variable (firm performance). The $\mathrm{R}$-square value has been resulting from the output of SmartPLS explains that, bu putting all the constructs together have the propensity of influencing the changes in DV by $35 \%$. 
Table 4.

Summary of Direct Hypothesis Testing

\begin{tabular}{llllcccccc}
\hline $\begin{array}{c}\text { Sr- } \\
\text { no }\end{array}$ & Hypothesis & Beta & SD & $\begin{array}{c}\text { T } \\
\text { Statistics }\end{array}$ & $\begin{array}{c}\text { P } \\
\text { Values }\end{array}$ & Decision & $\begin{array}{c}\text { Effect } \\
\text { Size }\end{array}$ & $\begin{array}{c}\text { Q2 } \\
\text { Square }\end{array}$ \\
\hline 1 & GS -> FP & -0.088 & 0.054 & 1.636 & 0.051 & supported & 0.023 & 0.183 & 0.352 \\
2 & GS -> UEM & 0.239 & 0.047 & 5.073 & 0.000 & supported & 0.071 & 0.147 & 0.253 \\
3 & MO -> FP & 0.069 & 0.064 & 1.065 & 0.143 & Not Supported & 0.006 & & \\
4 & MO - > UEM & 0.023 & 0.081 & 0.283 & 0.389 & Not Supported & 0.001 & & \\
5 & RA -> FP & 0.235 & 0.056 & 4.187 & 0.000 & supported & 0.057 & & \\
6 & RA -> UEM & 0.152 & 0.061 & 2.473 & 0.007 & supported & 0.027 & & \\
7 & TMS -> FP & 0.449 & 0.048 & 9.331 & 0.000 & supported & 0.185 & & \\
8 & TMS - > UEM & 0.329 & 0.060 & 5.520 & 0.000 & supported & 0.129 & & \\
9 & UEM -> FP & 0.180 & 0.057 & 3.135 & 0.001 & supported & 0.037 & & \\
\hline
\end{tabular}

\section{Mediation Model}

Resampling mediation-technique (bootstrapping) has been used in current study to examines the indirect effects of each variable. Similarly, most of the studies shown that "Bootstrapping is a non-parametric re-sampling procedure" and receiving more responsiveness because this technique is considered as most potential procedure to test and analyse the effect of mediation (Zhao et al., 2010; Hayes, 2009). Furthermore, as recommended by Hair et al. (2014) that, PLS (SEM) bootstrapping technique for mediation analysis is known as best suited for quatitative studies because it is appropriate for small samples as well. However, in line with the recommendation of Hair et al. (2014), at the time of doing analysis of mediation effects, one must follow the procedure of mediation as explained by Preacher and Hayes $(2004,2008)$ and bootstrap the distribution of sampling of in-direct effects which works for simple as well as for multiple models. Thus, this study has examined the influence of use of e-marketing as a mediating variable by using SmartPLS 3.0 (Ringle et al., 2015) using the bootstrapping technique by doing re-sampling of 500 to determine the $\mathrm{t}$ value. 


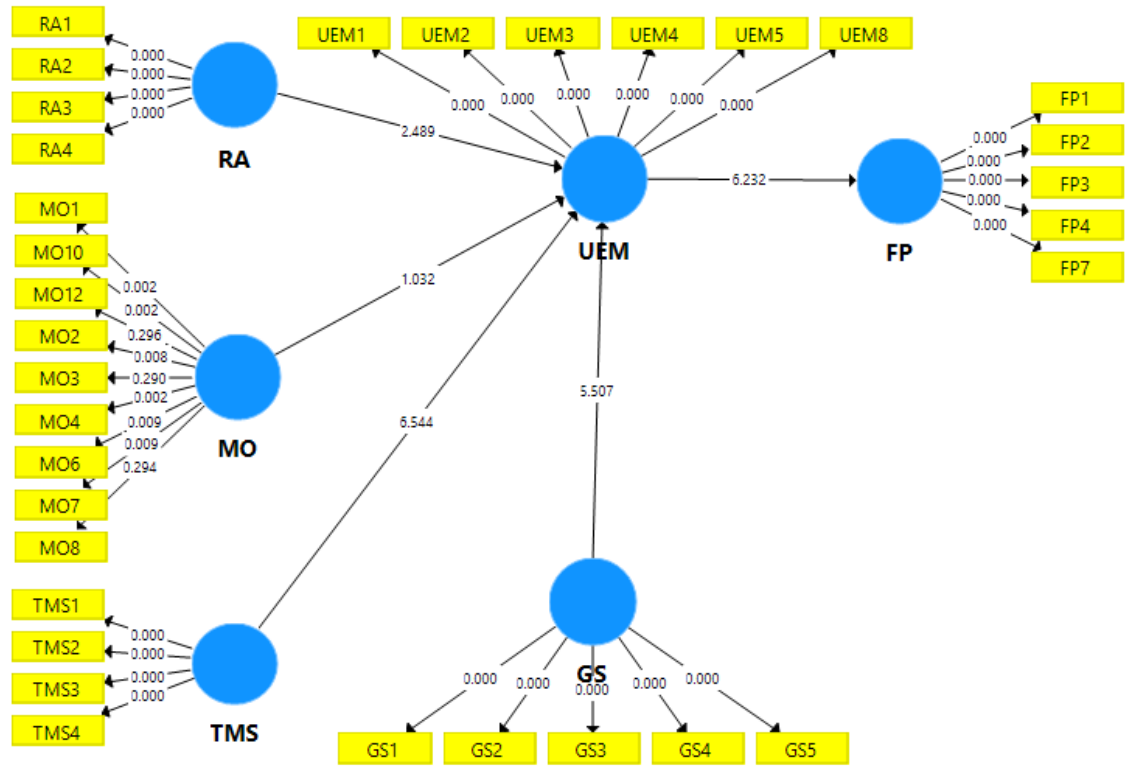

Figure 4. The Indirect Effect of Use of e-Marketing

In current study, after examining the assessment of structural model it has been found that, from four independent variables included in the structural model three independent variables which are competitive pressure, top management support and technology orientation were significantly associated with use of e-marketing and firm performance. However, Table 5 shows the results of mediation effect of use of e-marketing on the relationships between latent variables and dependent variable.

Table 5.

Mediaton Results

\begin{tabular}{lccccccc}
\hline Hypothesis & Beta & SD & $\begin{array}{c}\text { T } \\
\text { Stats }\end{array}$ & $\begin{array}{c}\text { P } \\
\text { Values }\end{array}$ & $\mathbf{5 . 0 \%}$ & $\mathbf{9 5 . 0 \%}$ & Results \\
\hline GS -> UEM ->FP & 0.0909 & 0.0179 & 5.0764 & 0.0000 & 0.0632 & 0.1220 & Mediation \\
MO -> UEM ->FP & 0.0346 & 0.0332 & 1.0401 & 0.1492 & -0.0443 & 0.0704 & No Mediation \\
RA -> UEM ->FP & 0.0507 & 0.0246 & 2.0564 & 0.0199 & 0.0188 & 0.0994 & Mediation \\
TMS -> UEM -> FP & 0.1247 & 0.0356 & 3.5003 & 0.0002 & 0.0778 & 0.1957 & Mediation \\
\hline
\end{tabular}


Lastly, table 5 explains that, three variables out of four shows mediation which includes government support with the t-value of 5.08, top management support with t-value of 3.50 and relative advantage with t-value 2.06, it shows significant partial mediation except Market orientation (MO) which become weak and insignificant after intervening of use of e-marketing as a mediator between the relationships.

\section{Predictive Relevance of the Model}

Moreover, to test the predictive relevance of the model, current study has used blindfolding procedure as given in SmartPLS 3.0. Therefore, to assess the predictive capacity of the model blindfolding procedure was considered. Predictive relevance is also known by the denotion of Q2. Furthermore, as explained by Hair et al. (2014) that Q-value is acquired by using the blindfolding "to assess the parameter estimates" and also assess "how values are built around the model". The results retrieved from blindfolding output through variable-score from which cross-validated redundancy has been extracted. These cross validated redundancy analysed the capacity of the model to predict the relevance of dependent variables and also explicates the quality of the overall model. Therefore, Table 6 describes the variable crossvalidated redundancy. Table 6 also reveals that in four (4) column, Q2 illustrates the predictive relevance of 0.183 for firm performance (FP) and 0.150 for use of e-marketing (UEM) that confirms the model predictive relevance.

Table 6.

Predictive Relevance

\begin{tabular}{cccc}
\hline Total & SSO & SSE & 1-SSE/SSO \\
\hline FP & 1285 & 1050 & 0.183 \\
UEM & 1542 & 1314 & 0.150 \\
\hline
\end{tabular}




\section{Discussion and Conclusion}

The purpose of this research is to investigate the mediating role of UEM (use of e-marketing) between technological, organizational, environmental factors and the performance of small and large firms in Pakistan. Contrariwise, this research paper has identified the link between e-marketing uses and the performance of firms working in Pakistan. The important hypotheses resulted that UEM mediates the relationship among its antecedents and the performance of businesses in Pakistan. The results established that UEM by the enterprises positively impact on firm performance; hence, it can be determined that higher the e-marketing use by the businesses, higher is the probability of distended corporate performance. The results relates with past literature which explains that e-marketing uses supports to drive business performance, which contingent that adoption and use of technology like e-marketing indicates opportunities for the business which are broadly reflected as a strong driver to attain business performance (Sheikh et al., 2016).

The results of this research explains that top management support, relative advantage and government support indirectly related with the business performance through use of e-marketing as a mediation, though, after analysis the hypotheses, the research argues that UEM mediates the association between technological, organizational and environmental factors excluding one factor which is market orientation resulted insignificant also concurred with past literature (Han, Kim \& Srivastava, 1998).

Awan \& Zhang (2013) and Mzee, Ogweno and Irene (2015) clarifies that electronic marketing is growing rapidly and becoming a essential tool for the businesses, although, this research has strained to examine the connotation among internet marketing uses and business performance in underdeveloped countries because there is still lack of empirical studies in this particular field. Also, e-marketing adoption to attain business performance in Pakistan is still on its infancy stage and factors such as relative advantage, top management support, market orientation and government support have yet to be explored. However, current IT infrastructure is not helpful in Pakistan to progress marketing performance, so in current situation, early adoption of technology is very important (Sheikh et al., 2016). 
The other main purpose of this study is to investigate the direct relationship between technological, organizational, environmental factors with the performance of businesses in Pakistan. Therefore, on the basis of direct relationships, the research results identified that relative advantage (RA), top management support (TMS) and government support (GS) has a noteworthy relationship with use of e-marketing (UEM). Furthermore, top management support and relative advantage is positively linked with firm performance. Besides, government support has revealed significant negative link with performance of businesses in Pakistan. Regrettably, MO has resulted insignificant both directly and indirectly by inclusion of mediation. Though, the insignificant result of market orientation concurred with past literature (Han et al., 1998; Shehu \& Mahmood, 2014).

Finally, this research has tried to fill the gap in the existing literature on firm performance and e-marketing usage, where prevailing clarifications have concentrated on the market characteristics, firm's technology through the awareness of e-marketing uses. Theoretically, the current research has contributed in the existing knowledge of literature and also by extending the related theories to increase the performance of businesses working in Pakistan. Conclusively, this research has enriched the literature by examining the relationship between UEM and performance of firms in dynamic process as well as in rapidly changing environment with the support of several independent variables. Besides, the practical contribution is concerned the conclusions of this research, probably facilitate the policy makers, industrialists, general manager marketing and management of manufacturing firms to develop technology related strategies more efficiently.

\section{Implications}

In terms of academic implications, the research can be considered unique in the field of e-marketing in general and e-marketing in small and large businesses of Pakistan in particular. This study provides empirical evidence for the theoretical relationships hypothesized in the research framework. Specifically, it highlights the mediating role of the use of e-marketing on the relationship between technological factor which is RA, organizational 
factors which are TMS and MO, environmental factor which is GS and their effect on performance of small and large businesses in Pakistan.

The first significant implication of this research in the e-marketing field is not only based on validating TOE model and DOI theory in the context of e-marketing adoption, but also the extension of these two to increase their ability to illustrate this adoption. This study proposed and examined the factors that affect the use of e-marketing by extending TOE and DOI to include factors that are more related to the performance of small and large businesses in Pakistan. However RBV is discussing about the tangible and intangible factors which are helping the firm to achieve better performance. Thus, the study helps researchers to have a deep understanding about the different relationships among RA, TMS, MO, GS with use of e-marketing as well as their relationship with small and large businesses performance directly and indirectly by use of e-marketing as a mediating variable. Consequently, it confirms that the use of e-marketing depends on the perceptions of corporate owners and marketing managers about its effectiveness.

Finally, employing the same logic and based on the same findings, as well as findings related to the current practices of e-marketing by Pakistan businesses, the Pakistan government through its ministries, agencies (Ministry of Textile, Pakistan, Small and medium enterprise development authority-SMEDA) can use the e-marketing adoption model developed within this study as well as its findings to increase the levels of e-marketing adoption, diffusion and practices by businesses to have a positive impact on Pakistan economy performance. Practically, this study has strength for managerial applications by using e-marketing in the system of manufacturing sector of Pakistan.

\section{Limitations and Future Recommendation}

Despite several significant contributions highlighted in this study regarding performance of businesses in Pakistan, it has several limitations that need to be identified. Firslty, the potential limitation of this study relates to the measures of the constructs used in this research work. The variables in this study were measured as a one-dimensional variable. However, variables of 
the current study can give more information if considered as multidimensional. Therefore, further investigation on the relationship between these variables and firm performance using multi-dimensional scale is a fertile area of research.

Secondly, the study adopted cross-sectional design for the survey in which the opinions of respondents was captured at one specific point in time. Thus, due to the cross-sectional nature of this study, it is restricted in proving causal relationships between the variables (Sekaran \& Bougie, 2010). As the data was collected at one time, this might not permit the data to represent long-term behaviors of the firms. In view of these restrictions, a longitudinal study is suggested for future research. This may help researchers get more understanding on the subjected matter and validate the findings from cross-sectional studies.

Thirdly, this study examined the mediating role of the use of e-marketing on the relationship between TOE factors and performance of businesses in Pakistan. The independent variables tested in the study were confined to performance. Other factors that belong to TOE factors can be used to extend the framework proposed in the study.

For future research, the current study adopted quantitative method and rely on a single method of data collection. In other words, the questionnaire was the only instrument used in gathering the data in this study. Thus, the responses may not consistently and accurately measure the study variables. It will be of interest if future studies combine both quantitative and qualitative methods to carry out an in-depth investigation on the performance and use of e-marketing in the industry of Pakistan.

\section{References}

Ahmad, S.Z., Bakar, A.R.A., Mohamed, T., Faziharudean, \& Zaki, K.A.M. (2014). Information Technology for Development An Empirical Study of Factors Affecting e-Commerce Adoption among Small- and Medium-Sized Enterprises in a Developing Country: Evidence from Malaysia. Information Technology For Development, 21(4), 37-41. doi: 10.1080/02681102.2014.899961 
Atuahene-Gima, K., \& Murray, J.Y. (2004). Antecedents and outcomes of marketing strategy comprehensiveness. Journal of Marketing, 68(4), 33-46. doi: 10.1509/jmkg.68.4.33.42732

Allen, D.G., Shore, L.M., \& Griffeth, R.W. (2003). The role of perceived organizational support and supportive human resource practices in the turnover process. Journal of Management, 29(1), 99-118. doi: 10.1177/014920630302900107

Al-Qirim, N. (2007). The adoption of eCommerce communications and applications technologies in small businesses in New Zealand. Electronic Commerce Research and Applications, 6(4), 462-473. doi: 10.1016/j.elerap.2007.02.012

Arifin, Z., \& Frmanzah. (2015). The Effect of Dynamic Capability to Technology Adoption and its Determinant Factors for Improving Firm's Performance; Toward a Conceptual Model. Procedia - Social and Behavioral Sciences, 207(2015), 786-796. doi: 10.1016/j.sbspro.2015.10.168

Awan, M.A., \& Zhang, W. (2013). Export Marketing Performance (EMP) of Pakistan SMEs impacted by the Internet. GSTF Journal on Business Review, 2(3), 71-79. doi: 10.5176/2010-4804_2.3.235

Barney, J. (1991). Firm Resources and Sustained Competitive Advantage. Journal of Management, 17(1), 99-120. doi: 10.1177/014920639101700108

Benitez-Amado, J., Llorens-Montes, F.J., \& Perez-Arostegui, M.N. (2010). Information technology-enabled intrapreneurship culture and firm performance. Industrial Management \& Data Systems, 110(4), 550566. doi: 10.1108/02635571011039025

Chen, C.C.-W., \& Lien, N.N.-H. (2013). Technological opportunism and firm performance: Moderating contexts. Journal of Business Research, 66(11), 2218-2225. doi: 10.1016/j.jbusres.2012.02.001

Cornford, T., \& Smithson, S. (2006). Project research in information systems: a student's guide.

Das, A., Majumdar, L., Chakrabarti, S., \& Chakrabarti, S. (2013). Chemical evolution during the process of proto-star formation by considering a two dimensional hydrodynamic model. New Astronomy, 23, 118-125. doi: 10.1016/j.newast.2013.01.003 
Deshpande, R., Farley, J.U., \& Webster Jr., F.E. (1993). Corporate culture, customer orientation, and innovativeness in Japanese firms: a quadrad analysis. The Journal of Marketing, 57(1), 23-37. doi: $10.2307 / 1252055$

Duan, X., Deng, H., \& Corbitt, B. (2012). What Drives the Adoption of Electronic Markets in Australian Small-and- Medium Sized Enterprises? - An Empirical Study. Australasian Conference on Information Systems (23rd : 2012 : Geelong, Victoria), 1-11.

Eid, R., \& El-Gohary, H. (2013). The impact of E-marketing use on small business enterprises' marketing success. The Service Industries Journal, 33(1), 31-50. doi: 10.1080/02642069.2011.594878

El Gohary, H. (2012). Factors affecting E-Marketing adoption and implementation in tourism firms: An empirical investigation of Egyptian small tourism organisations. Tourism Management, 33(5), 1256-1269. doi: 10.1016/j.tourman.2011.10.013

El Gohary, H. (2010). E-Marketing - A Literature Review From A Small Businesses Perspective. International Journal of Business and Social Science, 1(1).

El Gohary, H., Trueman, M., \& Fukukawa, K. (2008). E-Marketing and Small Business Enterprises: A review of the methodologies, 2(2), 6493. doi: 10.1080/02642069.2011.594878

Fink, D. (1998). Guidelines for the successful adoption of information technology in small and medium enterprises. International Journal of Information Management. doi: 10.1016/S0268-4012(98)00013-9

Fornell, C., \& Larcker, D.F. (1981). Evaluating structural equation models with unobservable variables and measurement error. Journal of marketing research, 39-50. doi: 10.2307/3151312

Fredriksson, T. (2013). E-commerce and Development Key Trends and Issues. In Workshop on E-Commerce, Development and SMEs (pp. 89).

George, D., \& Mallery, M. (2003). Using SPSS for Windows step by step: a simple guide and reference. Boston: Allyn \& Bacon.

Grandon, E.E., \& Pearson, J.M. (2004). Electronic commerce adoption : an empirical study of small and medium US businesses. Information \& Management, 42(1), 197-216. doi: 10.1016/j.im.2003.12.010 
Haider, Z., Chen, S., \& Abbassi, Z. (2015). Adoption of e-Government in Pakistan: Demand Perspective. International Journal of Advanced Computer Science and Applications, 6(5), 71-80. doi: 10.14569/IJACSA.2015.060512

Hair Jr, J.F., \& Lukas, B. (2014). Marketing research. McGraw-Hill Education Australia.

Hair, J.F., Black, W.C., Babin, B.J., Anderson, R.E., \& Tatham, R.L. (2010). Multivariate Data Analysis. Prentice Hall.

Han, J.K., Kim, N., \& Srivastava, R.K. (1998). Market Orientation and Organizational Performance Is Innovation a Missing Link. Journal of Marketing, 62(4), 30-45. doi: 10.2307/1252285

Hassan, M. ul, Mukhtar, A., Qureshi, S.U., \& Sharif, S. (2012). Impact of TQM practices on firm's performance of Pakistan's manufacturing organizations. International Journal of Academic Research in Business and Social Sciences, 2(10).

Haugh, H., \& Robson, P. (2005). Are Scottish firms meeting the ICT challenge? Results from a National Survey of Enterprise. Entrepreneurship \& Regional Development, 17(3), 205-222. doi: 10.1080/08985620500096711

Hayes, A.F. (2009). Beyond Baron and Kenny: Statistical mediation analysis in the new millennium. Communication monographs, 76(4), 408-420. doi: 10.1080/03637750903310360

Henseler, J., Ringle, C.M., \& Sinkovics, R.R. (2009). The use of partial least squares path modeling in international marketing. In New challenges to international marketing (pp. 277-319). Emerald Group Publishing Limited.

Hinkin, T. R. (1995). A review of scale development practices in the study of organizations. Journal of Management, 21(5), 967-988. doi:

10.1177/014920639502100509

Hooley, G.J., Greenley, G.E., Cadogan, J.W., \& Fahy, J. (2005). The performance impact of marketing resources. Journal of Business Research, 58(1), 18-27. doi: 10.1016/S0148-2963(03)00109-7

$\mathrm{Hu}$, Q., Wu, X., \& Wang, C.K. (2004). Lessons from Alibaba.com: government's role in electronic contracting. Info, 6(5), 298-307. doi: $10.1108 / 14636690410564807$ 
Hult, G., Hurley, R., \& Knight, G. (2004). Innovativeness: Its antecedents and impact on business performance. Industrial Marketing Management, 33(5), 429-438. doi: 10.1016/j.indmarman.2003.08.015

Iddris, F., \& Ibrahim, M. (2015). Examining the relationships between eMarketing adoption And Marketing Performance of Small and Medium Enterprises in. Journal of Marketing and Consumer Research, 10(2015), 160-170.

Ireland, R., \& Webb, J. (2007). A multi-theoretic perspective on trust and power in strategic supply chains. Journal of Operations Management, 25(2), 482-497. doi: 10.1016/j.jom.2006.05.004

Johnson, G., Scholes, K., \& Fréry, F. (2002). Stratégique, 2e éd. Paris: Pearson Education.

Johnston, D.A., Wade, M., \& McClean, R. (2007). Does e-business matter to SMEs? A comparison of the financial impacts of internet business solutions on European and North American SMEs. Journal of Small Business Management, 45(3), 354-361. doi: 10.1111/j.1540627X.2007.00217.x

Johnston, D., \& Wright, L. (2004). The e-business capability of small and medium sized firms in international supply chains. Information Systems and E-Business Management, 2(2-3), 223-240. doi: $10.1007 / \mathrm{s} 10257-004-0038-2$

Junaid, A. (2015, October 19). The encouraging future of e-commerce in Pakistan. The Express Tribune. Retrieved from http://tribune.com.pk/story/975430/the-encouraging-future-of-ecommerce-in-pakistan

Kazungu, I., \& Panga, F.P. (2015). Impediments To Adoption of EMarketing By Tanzanian Small and Medium Sized Enterprises : an Explanatory Model. International Journal of Economics, Commerce and Management, III(6), 587-597.

Kotler, P., \& Keller, K. (2006). Administração de marketing. Sao Paolo: Pearson-Prentice Hall.

Li, Y.H. (2008). An empirical investigation on the determinants of Eprocurement adoption in Chinese manufacturing enterprises. 2008 International Conference on Management Science and Engineering 15th Annual Conference Proceedings, ICMSE, 32-37. 
Lopez-Acevedo, G. (2002). Technology and firm performance in Mexico. Retrieved from the World Bank website: http://documents.worldbank.org/curated/en/655361468753009855/Te chnology-and-firm-performance-in-Mexico

Lucia-Palacios, L., Bordonaba-Juste, V., Polo-Redondo, Y., \& Grünhagen, M. (2013). Technological opportunism effects on IT adoption, intrafirm diffusion and performance: Evidence from the U.S. and Spain. Journal of Business Research, 67(6), 1178-1188. doi: 10.1016/j.jbusres.2013.05.004

Mącik, R., Jóźwik, B., \& Nalewajek, M. (2012). E-Marketing Activities and Perceived Competitive Advantage in the Context of Globalization: Study of Lublin Region Firms. In In Knowledge and Learning: Global Empowerment; Proceedings of the Management, Knowledge and Learning International Conference 2012 (pp. 741-749). International School for Social and Business Studies, Celje, Slovenia.

Mackey, A. (2008). The Effect of CEOs on Firm Performance. Strategic Management Journal, 29(12), 1357-1367. doi: 10.1002/smj.708

Markides, C. (1997). Strategic innovation. MIT Sloan Management Review, 38(3), 9.

Mohamad, R., Building, A., \& Ismail, N.A. (2009). Electronic Commerce Adoption in SME: The Trend of Prior Studies. Journal of Internet Banking and Commerce, 14(2), 1-16.

Molla, A., \& Licker, P.S.P. (2005). eCommerce adoption in developing countries: a model and instrument. Information \& Management, 42(6), 877-899. doi: 10.1016/j.im.2004.09.002

Morais, M. (2006). e-business in textile industry. emarketservices (Vol. XXXIII).

Mutlu, H.M., \& Sürer, A. (2015). Effects of Market, E-Marketing, and Technology Orientations on Innovativeness and Performance in Turkish Health Organizations. Health Marketing Quarterly, 32(4), 313-329. doi: 10.1080/07359683.2016.1166807

Mzee, R.M., Ogweno, K.L., \& Irene, N. (2015). Factors influencing Adoption of E marketing by small and Medium Enterprises (SMEs) in Kisumu Municipality. IOSR Journal of Business and Management, 17(5), 78-83. doi: 10.9790/487X-17537883 
Narver, J.C., \& Slater, S.F. (1990). The effect of a market orientation on business profitability. The Journal of marketing, 54(4) 20-35. doi: $10.2307 / 1251757$

Narver, J.C., Slater, S.F., \& MacLachlan, D.L. (2000). Total Market Orientation, Business Performance, and Innovation. Retrieved from Marketing Science Institute website: http://www.msi.org/reports/totalmarket-orientation-business-performance-and-innovation/

Noshab, F. (2006). Globalization, WTO and Pakistan. The Muslim World, 96(2), 341-262. doi: 10.1111/j.1478-1913.2006.00132.x

O'Mahony, M., \& Van Ark, B. (Eds.) (2003). EU productivity and competitiveness: An industry perspective. Can Europe resume the catching-up process? Luxembourg: Office for Official Publications of the European Communities.

PACRA (2011). Sector Study. Textile Sector FY11. Retrieved from the Pakistan Credit Rating Agency Limited website: http://www.pacra.com.pk/pages/research/web_sector_study/textile/Te xtile_Mar_11.pdf

Pakistan, E.S.O. (2015). Overview of the Economy. Retrieved from http://finance.gov.pk/survey/chapters_15/Overview_of_the_Economy. pdf

Perrow, C., Thompson, J.D., \& Peterson, R.A. (1970). Departmental power and perspectives in industrial firms. Power in Organizations, 7(1970), 58-89.

Porter, M. (1985). Competitive advantage: Creating and sustaining superior performance. New York: The Free Press.

Porter, M. (1990). The competitive advantage of nations. Harvard Business Review.

Prasad, V.K., Ramamurthy, K., \& Naidu, G.M. (2001). The Influence on of Internet-Marketing Marketing Performance Competencies Integration and Export. Journal of International Marketing, 9(4), 82-110. doi: 10.1509/jimk.9.4.82.19944

Preacher, K.J., \& Hayes, A.F. (2004). SPSS and SAS procedures for estimating indirect effects in simple mediation models. Behavior research methods, instruments, \& computers, 36(4), 717-731. doi: 10.3758/BF03206553 
Preacher, K.J., \& Hayes, A.F. (2008). Asymptotic and resampling strategies for assessing and comparing indirect effects in multiple mediator models. Behavior research methods, 40(3), 879-891. doi: 10.3758/BRM.40.3.879

Premkumar, G., \& Roberts, M. (1999). Adoption of new information technologies in rural small businesses. Omega: The International Journal of Management Science, 27(4), 467-484. doi: 10.1016/S03050483(98)00071-1

Premkumar, G., Ramamurthy, K., \& Nilakanta, S. (1994). Implementation of electronic data interchange: an innovation diffusion perspective. Journal of Management Information Systems, 11(2), 157186. doi: 10.1080/07421222.1994.11518044

Qureshi, S., Keen, P., \& Kamal, M. (2010). Business models for development: The global capability sourcing model. In Sherif Kamel (Ed.), E-Strategies for Technological Diffusion and Adoption: National ICT Approaches for Socioeconomic Development (pp. 200214). IGI Publishing.

Rahayu, R., \& Day, J. (2015). Determinant Factors of E-commerce Adoption by SMEs in Developing Country: Evidence from Indonesia. Procedia - Social and Behavioral Sciences, 195(2015), 142-150. doi: 10.1016/j.sbspro.2015.06.423

Rahim, A., Bakar, A.B.U., \& Ahmed, Z.U. (2015). Technology Motivation in E-Marketing Adoption Among Malaysian Manufacturers. Journal of Transnational Management, 20(April), 126-152. doi: 10.1080/15475778.2015.1038949

Raymond, L., Bergeron, F., \& Blili, S. (2005). The Assimilation of Ebusiness in Manufacturing SMEs: Determinants and Effects on Growth and Internationalization. Electronic Markets, 15(2), 106-118. doi: 10.1080/10196780500083761

Rayport, J., \& Jaworski, B. (2004). Best face forward. Harvard Business Review, 82(12), 47-59.

Riddell, W., \& Song, X. (2012). The role of education in technology use and adoption: Evidence from the Canadian workplace and employee survey. ILR Review. doi: 10.1177/0019793916687719 
Ringle, C.M., Wende, S., \& Becker, J.-M. (2015). "SmartPLS 3." Boenningstedt: SmartPLS GmbH, http://www.smartpls.com.

Rusli, E. (2012). Facebook buys Instagram for $\$ 1$ billion. New York Times.

Sabbaghi, A., \& Vaidyanathan, G. (2008). Effectiveness and efficiency of RFID technology in supply chain management: strategic values and challenges. Journal of Theoretical and Applied Electronic Commerce Research, 3(2), 71-81. doi: 10.4067/S0718-18762008000100007

Sam, T., \& Leng, A. (2006). Adoption of electronic commerce amongst small and medium enterprise. In In Knowledge Management International Conference and Exhibition.

Schumpeter, J. (1934). The theory of economic development: An inquiry into profits, capital, credit, interest, and the business cycle (Vol.55). Transaction publishers.

Sekaran, U., \& Bougie, R. (2010). Research methods for business: A skill building approach. London: John Wiley \& Sons.

Seyal, A. H., Awais, M. M., Shamail, S., \& Abbas, A. (2004). Determinants of Electronic Commerce in Pakistan: Preliminary Evidence from Small and Medium Enterprises. Electronic Markets by Taylor \& Francis, 14(4), 372-387. doi: 10.1080/10196780412331311801

Shah, W., Warraich, U.A., \& Kabeer, K. (2012). Challenges Faced by Textile Industry of Pakistan: Suggested Solutions. KASBIT Business Journal, 5(2012), 33-39.

Shaher, Y.A. (2012). Strategies For Pakistan Textiles Industry To Sustain The Business. University of Gavle.

Shehu, A.M., \& Mahmood, R. (2014). Market orientation and firm performance among nigerian SMEs: The moderating role of business environment. Mediterranean Journal of Social Sciences, 5(23), 158164. doi: 10.5901/mjss.2014.v5n23p158

Sheikh, A.A., Shahzad, A., \& Ishak, A.B.K. (2016). The mediating impact of e-marketing adoption on export performance of firms: A conceptual study. Journal of Technology and Operations Management, 11(1), $48-58$.

Srinivasan, R., Lilien, G.L., \& Rangaswamy, A. (2002). Technological Opportunism and Radical Technology Adoption: An Application to E- 
Business. Journal of Marketing, 66(3), 47-60. doi: 10.1509/jmkg.66.3.47.18508

Straus, J., \& Frost, R. (2001). E-marketing. New Jersey: Prentice Hall Tam, K.Y. (1998). The Impact of Information Technology Investments on Firm Performance and Evaluation: Evidence from Newly Industrialized Economies. Information Systems Research, 9(1), 85-98. doi: $10.1287 /$ isre.9.1.85

Tan, M., \& Teo, T. (1998). Factors influencing the adoption of the Internet. International Journal of Electronic Commerce, 2(3), 5-18. doi: 10.1080/10864415.1998.11518312

Tandon, N., \& Reddy, E.E. (2013). A Study on Emerging Trends in Textile Industry in India. AMET International Journal of Management, 8188(7), 267-276.

Teo, T. (2007). Organizational characteristics, modes of internet adoption and their impact: a Singapore perspective. Journal of Global Information Management, 15(2), 91. doi: 10.4018/jgim.2007040104

Teo, T., \& Pian, Y. (2003). A contingency perspective on Internet adoption and competitive advantage. European Journal of Information Systems, 12(2), 78-92. doi: 10.1057/palgrave.ejis.3000448

Thatcher, S.M.B., Foster, W., \& Zhu, L. (2006). B2B e-commerce adoption decisions in Taiwan: The interaction of cultural and other institutional factors. Electronic Commerce Research and Applications, 5(2), 92104. doi: $10.1016 /$ j.elerap.2005.10.005

Thompson, V. (1965). Bureaucracy and innovation. In Administrative science quarterly (pp. 1-20).

Thong, J., \& Yap, C. (1995). CEO characteristics, organizational characteristics and information technology adoption in small businesses. Omega, 23(4), 429-442. doi: 10.1016/03050483(95)00017-I

Ueasangkomsate, P. (2015). Adoption E-Commerce for Export Market of Small and Medium Enterprises in Thailand. Procedia - Social and Behavioral Sciences, 207, 111-120. doi: 10.1016/j.sbspro.2015.10.158

Varukolu, V. (2007). Technology adoption of india garment manufacturing firms. Doctoral dissertation, Faculty of the Louisiana State University 
and Agricultural and Mechanical College in partial fulfillment of the requirements for the degree of Master of Science in The School.

Voola, R., Casimir, G., Carlson, J., \& Anushree Agnihotri, M. (2012). The effects of market orientation, technological opportunism, and ebusiness adoption on performance: A moderated mediation analysis. Australasian Marketing Journal, 20(2), 136-146. doi: 10.1016/j.ausmj.2011.10.001

WTO (2014). International Trade Statistics 2014.

Wang, Y.M., Wang, Y.S., \& Yang, Y.F. (2010). Understanding the determinants of RFID adoption in the manufacturing industry. Technological Forecasting and Social Change, 77(5), 803-815. doi: 10.1016/j.techfore.2010.03.006

Woodward, J., Dawson, S., \& Wedderburn, D. (1965). Industrial organization: Theory and practice (Vol. 3). London: Oxford University Press.

Wu, F., Mahajan, V., \& Balasubramanian, S. (2003). An Analysis of EBusiness Adoption and its Impact on Business Performance An Analysis of E-Business Adoption and its Impact on Business Performance. Journal of the Academy of Marketing Science, 31(April 2003), 425-447. doi: 10.1177/0092070303255379

Zhao, X., Lynch, J.G., \& Chen, Q. (2010). Reconsidering Baron and Kenny: Myths and truths about mediation analysis. Journal of consumer research, 37(2), 197-206. doi: 10.1086/651257

Adnan Ahmed Sheikh is $\mathrm{PhD}$ candidate Othman Yeop Abdullah at the Graduate School of Business, Universiti Utara Malaysia

Arfan Shahzad is Senior Lecturer of Othman Yeop Abdullah at the Graduate School of Business, Universiti Utara Malaysia

Awanis Ku Ishak is Senior Lecturer at the School of Business Management, Universiti Utara Malaysia

Contact address: Othman Yeop Abdullah Graduate School of Business (OYAGSB), Universiti Utara Malaysia, 06010 UUM Sintok, Kedah Darul Aman, Malaysia. Email: adnanucp@gmail.com 


\section{RIMCIS}

\section{Hipatia Press}

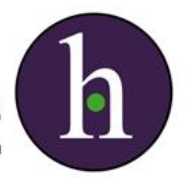

Instructions for authors, subscriptions and further details:

http://rimcis.hipatiapress.com

\section{How Solidarity Influences Political Actors to Manage the Refugee Crisis: The Case of Proactiva Open Arms}

Gemma Álvarez-Jiménez ${ }^{1}$, Maria Padrós-Cuxart ${ }^{1}$

1) Universidad de Barcelona, España

Date of publication: July $30^{\text {th }}, 2017$

Edition period: July 2017 - November 2017

To cite this article: Álvarez-Jiménez, G., \& Padrós-Cuxart, M. (2017). How Solidarity Influences Political Actors to Manage the Refugee Crisis: The Case of Proactiva Open Arms. International and Multidisciplinary Journal of Social Sciences, 6(2), 215-229. doi: 10.17583/rimcis.2017.2794

To link this article: http://doi.org/10.17583/rimcis.2017.2794

\section{PLEASE SCROLL DOWN FOR ARTICLE}

The terms and conditions of use are related to the Open Journal System and to Creative Commons Attribution License (CC-BY). 


\section{How Solidarity Influences Political Actors to Manage the Refugee Crisis: The Case of Proactiva Open Arms}

Gemma Álvarez-Jiménez

Universidad de Barcelona
Maria Padrós-Cuxart

Universidad de Barcelona

\section{Abstract}

Europe is facing the worst humanitarian crisis since World War II and the Mare Nostrum has become the path that millions of people are using to flee from the armed conflict, especially since the Syrian war began in March 2011. In this sense, according to the United Nations High Commissioner for Refugees (UNHCR), $1,014,973$ people arrived to Greece, Italy and Spain by sea in 2015 . Nevertheless, the Mediterranean area has become the scene of not only a humanitarian crisis but also citizens' solidarity. In this sense, Proactiva Open Arms, an NGO mostly formed by lifeguards, has helped 135,000 people to reach the coast safely, 10,273 of whom sailed in wandering boats. Of these individuals, 475 fell into the water and 9,067 were trapped on the cliffs. Proactiva Open Arms' work has driven European and Greek authorities to respond to refugees. In this paper, we analyse through this case how solidarity among civil citizens can change political actions and encourage other citizens to act in solidarity, as part of the H2020 European funded research project SOLIDUS

Keywords: solidarity, refugee crisis, social impact, human rights, citizen's initiative 


\section{Cómo Influye la Solidaridad en los Actores Políticos que Gestionan la Crisis de los Refugiados: El Caso de Proactiva Open Arms}

Gemma Álvarez-Jiménez Universidad de Barcelona
Maria Padrós-Cuxart

Universidad de Barcelona

\section{Resumen}

Europa se enfrenta a la peor crisis humanitaria desde la Segunda Guerra Mundial y el Mar Mediterráneo se ha convertido en el camino que millones de personas están utilizando para huir del conflicto armado, especialmente desde que la guerra siria comenzase en marzo de 2011. En este sentido y según el Alto Comisionado de las Naciones Unidas para los Refugiados (ACNUR), 1.014.973 personas llegaron a Grecia, Italia y España por la vía marítima en 2015. Sin embargo, el área del Mediterráneo no sólo se ha convertido en el escenario de una crisis humanitaria, sino también de la solidaridad ciudadana. En este sentido, Proactiva Open Arms, una ONG formada en su mayoría por socorristas, ha ayudado a 135.000 personas a llegar a la costa de manera segura, 10.273 de los cuales navegaron en botes errantes. De estos individuos, 475 cayeron en el agua y 9,067 estaban atrapados en acantilados. El trabajo de Proactiva Open Arms ha llevado a las autoridades europeas y griegas a intensificar su respuesta ante los refugiados. En este trabajo analizamos a través de este caso cómo la solidaridad entre los ciudadanos civiles puede cambiar las acciones políticas y animar a otros ciudadanos a actuar solidariamente, como parte del proyecto de investigación financiado por fondos europeos H2020 SOLIDUS.

Palabras clave: solidaridad, crisis de refugiados, impacto social, derechos humanos, iniciativas ciudadanas

2017 Hipatia Press

ISSN: 2014-3680

DOI: $10.17583 /$ rimcis.2017.2794 


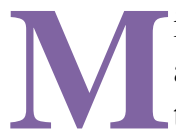

illions of people are fleeing from armed conflicts in Syria, Iraq and Afghanistan as well as other countries. Solely as a result of the Syrian civil war, according to the United Nations High Commissioner for Refugees (hereafter UNHCR) (2016a), 4,843,344 people have become refugees since its start in March 2011. Thus, trying to find shelter, refugees have moved to neighbouring countries such as Lebanon (1,048,275 refugees), Jordan (655,217 refugees), Iraq (246,589 refugees), Egypt (120,491 refugees) and Turkey (2,743,476 refugees), which are bearing the brunt of this humanitarian crisis and taking in the largest number of refugees (UNHCR, 2016a). However, Europe has also been affected by this migration crisis. Many refuges, attracted by the idea of safety in Europe, have started a long journey through land and sea routes with the aim of finding a safe place to rebuild their lives. According to UNHCR (2016b), $1,014,973$ people arrived to Greece, Italy and Spain by sea in 2015. The right to asylum is crucial for their salvation (Mautz, 2015). Thus, according to the Red Cross (2016), from January 2015 to June 2016, 1,221,277 refugees arrived in Europe, primarily through Greece and Italy. The sea route has become more important, especially given that some countries in the European Union started to block the land routes. One of the points of arrival of refugees prior to the EU-Turkish Agreement in March 2016 (Council of the European Union, 2016; European Commission, 2016) was Lesbos Island. This small Greek island is located $9 \mathrm{~km}$ off the coast of Turkey and it mainly dedicated to tourism. Its 85,000 inhabitants have been affected by the arrival of migrants and refugees since the summer of 2015 (Spanish Commission for Refugee Aid, 2016, p. 3). According to the Spanish Commission for Refugee Aid (2016), approximately 500,000 refugees arrived in Lesbos in 2015, particularly from the northeast region of Canakkale.

In this sense, also according to the Spanish Commission for Refugee Aid (2016), the Turkey-Lesbos routes started when refugees arrived at Istanbul and made their first contact with the traffickers in Taksim Square. The traffickers offered wealthy refugees a false passport and flights to reach Europe. However, those who could not afford to pay for a false passport and plane ticket were pushed to reach Europe via the Aegean Sea. As a result, these refugees went to the coastal city of Izmir, where traffickers received 


\section{8 Álvarez-Jiménez \& Padrós-Cuxart-Proactiva Open Arms}

them in Basmane Square. When the refugees arrived at that square, the traffickers took them to hostels in which they were hidden for approximately two weeks until they were transported by van to the point at which the boats sailed to Lesbos. The refugees paid approximately 1,000 to 2,000 euros per person to get onto the boats, according to Proactiva Open Arms. Although the traffickers offered them a false life vest without any safety guarantee, the refugees had to pay anyway. Thus, they began a dangerous journey on which many people lost their life. According to the Missing Migrants Project (IOM, 2016), 2,920 of them disappeared or died. According to the Red Cross (2016), the number is 2,856.

Given this situation, a group of volunteers, most of whom were professional lifeguards, who were members of the NGO Proactiva Open Arms, helped 135,000 people to reach the coast safely, 10,273 of whom had sailed in wandering boats. Of these individuals, 475 of whom had fallen into the water and 9,067 of whom were trapped on cliffs (these data was provided by Proactiva Open Arms showed in Clam Festival, 2016). These volunteers risked their own life to save many parents, children and elderly people who struggled to save the only thing that they had: their life. As a result, the European Parliament gave them the award for European Citizen's 2016 to recognise the impact of their caring work in Lesbos (European Parliament Affairs, 2016).

The social impact achieved by this NGO, mainly in terms of saving people's lives, but also in raising awareness among citizens and authorities (see Proactiva Open Arms website) about the alarming situation in the Mediterranean, and actually engaging them into action, motivated the SOLIDUS "SOLIDUS. Solidarity in European Societies: Empowerment, Social Justice and Citizenship"- funded by the European Commission under the Horizon 2020 Programme- to study this case further in depth. The objective of SOLIDUS is to analyse actions of solidarity in Europe in times of crisis that have achieved social improvements in order to inform public policy. 


\section{Data Collection \& Analysis}

The case study of Proactiva Open Arms was carried out through documental analysis of online information about this NGO, database from other organizations involve with refugees and press. Particularly, press is one of the most relevant communication channels of Proactiva Open Arms and this is the reason because we have collected it. Parallel, we have carried out qualitative interviews (two) with the goal of obtaining deeper information of the NGO Proactiva Open Arms. Given that Proactiva Open Arms team changes regularly, we selected two interviewers who were involved in the rescue of refugees since the beginning of the formation of this NGO until now. The interviews were made in May 2016. All of the names and personal information have been carefully treated and only used for the purpose of this article and SOLIDUS project, according to the agreement that was established by both parties involved. In addition, all real names have been anonymized and fictitious names have been created.

Table 1.

Interviews profiles

\begin{tabular}{lll}
\hline Interview & Gender & Profession
\end{tabular}

\begin{tabular}{lll}
\hline E1. Oriol & Male & $\begin{array}{l}\text { Lifeguard (Proactiva } \\
\text { Open Arms member) }\end{array}$ \\
E2. Jordi & Male & $\begin{array}{l}\text { Lifeguard (Proactiva } \\
\text { Open Arms member) }\end{array}$ \\
\hline
\end{tabular}

\section{Data analysis}

Documentary analysis and the (two) in-depth interviews has been analysed through biography technique. This technique have achieved great scientific relevance in qualitative research (Denzin, 1989, 2006; Denzin \& Lincoln, 2005). Concretely, we have made a biography of the NGO Proactiva Open 
Arms, remarking these situations where their solidarity action have transformed political actions and it has encouraged citizens' solidarity.

\section{Proactiva Open Arms Shows How Solidarity Can Transform Political Actions and Encourage Citizens' Solidarity}

The solidarity action of Proactiva Open Arms started when two lifeguard of Badalona (a municipality of Catalonia), who worked in a marine services company specializing in aquatic safety called Pro-Activa Servicios Acuáticos [Pro-Activa Water Services], decided to go to the island of Lesbos as a volunteers to help refugees. Thus, they arrived to the island of Lesbos in September 2015 with $€ 15,000$ to help refugees for a month (Pérez, 2016). In that time, Greece was overwhelmed by the situation (Kousoulis, IoakeimIoannidou \& Economopoulos, 2016), especially the island of Lesbos. The refugees' arrivals had increased exponentially (see figure 1), only in September 89, 690 refugees reached the island.

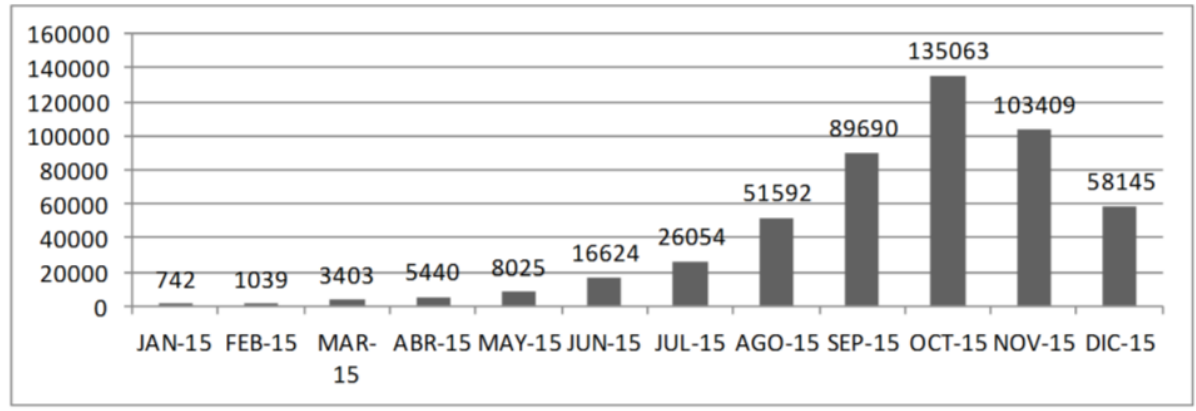

Source: UNHCR, 2015

Figure 1. Lesbos arrivals per month

Despite the hundreds of arrivals, no large NGO was receiving refugees on the coast. Only private citizens went there voluntarily to welcome refugees. Citizens are often the first to mobilise in crisis situations (Ataç, Rygiel \& Stierl, 2016; Hein \& Niazi, 2016). When the two lifeguard volunteers arrived and observed the situation with their own eyes, they were shocked. Many refugees drowned before reaching the shore because their 
boat sank due to overloading. No one, including the volunteers on the coast, swam to rescue the people who were sinking in the sea a few metres from the coast. Given the situation, the two lifeguard volunteers jumped into the seawater clothed to start to save people. However, instead of helping them, other volunteers started to shout at them. This situation was explained by one of the volunteers: "[other] volunteers, people like you and me who were as concerned as us, shouted at us saying that it was dangerous, what we were doing" (Oriol, Proactiva Open Arms member). Thus, instead of being supported and assisted by the rest of the volunteers, the two lifeguard volunteers were viewed as "crazy people"; however, they continued risking their lives to save others'. This is an example of how voluntary initiatives within solidarity movements can create barriers that impede the promotion of greater caring and improve their actions. In this sense, the question regarding why there are barriers within solidarity movements is interesting. One hypothesis could be that within these solidarity movements, the initial objective of their actions sometimes is forgotten, as the urgent focus on everyday tasks exceeds them. Thus, when an external initiative introduces a new method or means to achieve the objective, they not assess the effectiveness of the action in relation to the objective. Whereas this could be the case of the rest of the volunteers in relation to the Pro-Activa volunteers, deeper scientific analysis is necessary. In the case that is analysed in this paper, the Pro-Activa volunteers had a clear objective: to defend the right to life, the first law of human rights. Thus, Pro-Activa volunteers, despite this lack of caring and support from other volunteers who were on the coast, decided to wear lifeguard uniforms and continue to save lives. They decided to wear the uniform to show that they were professionals; therefore, they legitimised their job.

As a result of their work, they became indispensable to the island and obtained the support of the rest of volunteers. Thus, one of the volunteers explained, "Volunteers asked us for our phone and, when something occurred somewhere, they called us" (Jordi, Proactiva Open Arms member). However, a few months later the two volunteers of Pro-Activa run out their $€ 15,000$. In this situation, they decided to create a NGO, namely Proactiva Open Arms, and started a "crowdfunding" initiative. As a consequence of Proactiva Open Arms work on the island, they deserved the international 


\section{2 Álvarez-Jiménez \& Padrós-Cuxart-Proactiva Open Arms}

media's attention. This international media's attention allowed that crowdfunding initiative increased their resources exponentially. Thus, they increased their human resources, growing to 18 volunteers who rotated every 15 days in the period of maximum activity. In the beginning, they only had their personal equipment (neoprene suits, rubber rings, etc.). However, a few months later, they managed to obtain two cars, jet skis and three rescue boats. As it mentioned above, they started with a budget of $€ 15,000$ in 2015 but in 2016, up to September 30th, contributions reached 2.1 million euros (Proactiva Open Arms, 2016).

Additionally, they became indispensable to the Greece Authorities and Frontex as well, which originally did not coordinate with them or any other NGOs that were taking action on the island. As a consequence of the magnitude of the tragedy, political authorities started to contact them. In this sense, the night of October 28, 2015 is remembered as one of the worst nights at Lesbos Island. An old wooden boat that was in poor condition (the ship's hull was rotten) and overloaded, with three hundred people aboard approximately, broke and began to sink near the coast of Lesbos. At that time, NGO Proactiva Open Arms had 4 volunteers and only two jet skis for rescuing purposes. When they arrived at the wreck, they observed many people in the sea who were trying to save their lives. Some of them had died, and their bodies were floating in the sea. There were children, pregnant women, teenagers, men and women on board. Children and pregnant women were the most vulnerable groups on the boat. Typically, women and children cannot swim, so they are normally in a much worse situation than the rest of the group. Their situation were even worse than that of the others' due to the heavy wet clothes they wore that made them drown quickly.

Proactiva Open Arms was trying to save as many people as possible, but they soon realised that they needed help to overcome this situation. They could not cope with everything that was needed in this humanitarian crisis. Then, Frontex authorities went to the location to help refugees. However, Frontex's assistance was not as useful as expected. Frontex brought a large boat that was not equipped for rescuing services; it was designed for monitoring tasks. The boat had very high sides, making it difficult for the rescued refugees to get on board. In addition, the ship did not have medical services, and the Frontex professionals who were on the boat did not have 
enough knowledge to complete resuscitation tasks, according to Proactiva Open Arms. Given this lack of resources and knowledge, some Proactiva volunteers boarded the boat to save refugees and carry out resuscitation tasks (Diez \& Fontseca, 2016). At the same time, the task of the Greek Coastguard was also insufficient to address the tragedy. Thus, a group of Greek fishermen took their boats, that they used to perform their daily fishing activities, to rescue refugees. Finally, according to Proactiva Open Arms, approximately two hundred people were rescued, whereas many died, including mothers who left orphaned children. Proactiva Open Arms received the Honouring Excellence in Rescue Operations (HERO) award from the International Maritime Rescue Federation for their humanitarian work on October 28, 2015 (La Vanguardia, 2016). Parallel, two Greece volunteers, who also helped refugees, were nominated for the Nobel Peace Prize in 2016 (Schoenbauer, 2016).

The night of October 28, 2015 was not the only night in which Frontex or Greece authorities had to intervene. Nevertheless, it represented a turning point in the response by political authorities, especially Frontex and the Greece Coastguard. Since then, these authorities started to collaborate more intensely with the rest of the NGOs that were operating in Lesbos and performed rescue tasks in emergency situations. This change in response was a consequence of the example shown by Proactiva Open Arms. In this sense, one of the volunteers said, "When the Coastguard, Frontex and the UN overstated it, our presence humanised them" and added that "our presence forced them to act [...] even the Greek press reported that Proactiva Open Arms is humanising the Greece Coastguard. Our presence there made them work a little more, because we recorded them with cameras, and they knew it. Being internationally recognised, they could not avoid it" (Oriol, Proactiva Open Arms member). This is an example of how solidarity forces authorities to perform in a refugee crisis. The task of Proactiva Open Arms in Lesbos was widespread through several mass media outlets from Spain, Greece and even in the United States. The following headline was included in The New York Times: "As More Children Drowned, Volunteers on Lesbos Say Rescues Are Left Largely to Them" (Mackey, 2015).

After the EU-Turkey agreement in March 2016, the influx of refugees to the island of Lesbos decreased drastically. Thus, a volunteer said that 


\section{4 Álvarez-Jiménez \& Padrós-Cuxart-Proactiva Open Arms}

"arrivals are testimonial" (Jordi, Proactiva Open Arms member). However, this decrease in the number of refugees on the coast of Lesbos did not mean that refugees stopped trying to reach Europe. New routes through the Mediterranean emerged. Thus, North African routes became prevalent since March 2016, and traffickers continued their filthy commerce with refugees. The volunteers of Proactiva Open Arms, who was aware of this situation, continued to maintain solidarity with refugees. In this sense, in summer of 2016, they started to operate with a large boat called "Astral", which was donated by a multimillionaire for rescuing refugees in the area of Lampedusa. The boat was object of a remodelling. Thus, volunteers of Proactiva Open Arms worked hard to convert a luxury boat to a rescue boat in order to help refugees in the middle of the Mediterranean sea. Thus, volunteers made task of remodeling such as eliminate luxury objects, incorporate a cabin-infirmary, equip technology and improve communication systems to be able to collaborate with other organizations in the sea (Arias \& Palacios, 2016). Finally, in the mid-June 2016, the boat "Astral" sailed to the central Mediterranean in order to save more refugees (E1 Periódico, 2016). Since then, a team of volunteers formed by doctors, ship captain and lifeguards have been rescuing day and night hundreds of refugees. Only three months later, Proactiva Open Arms had already saved 12,500 refugees on the route of the central Mediterranean (Arias \& Palacios, 2016).

However, Proactiva Open Arms was mindful that the sailboat "Astral" was insufficient to save the refugees and brought them to the coast of Italy, thus they achieved a new bigger boat thanks to the solidarity of Grupo Ibaizabal, which is a commercial organisation involved primarily in the ship management. The boat donated by Grupo Ibaizabal was built in 1974 and it is a tugboat of $37 \mathrm{~m}$ length. That boat belonged to the Spanish Maritime Rescue Fleet along years. Now, it has been renamed as "Open Arms" tugboat. Such as the "Astral", this tugboat also has been remodeled in order to incorporate all the necessary tools to make humanitarian surveillance and rescuing tasks. In this new boat, there are 6 crew and more voluntaries. The tugboat has capacity to harbor 400 people and sailed away the summer of 2017. 
Proactiva Open Arms still saving the lives of refugees from the sea. Their labor started in September of 2015 from the solidarity of two lifeguards who decided to go to Lesbos with their own resources in order to help refugees. Thanks to the solidarity of other citizens, they have been able to continue their rescue work and increasing their infrastructures. Therefore, speaking of refugees supposes speaking about solidarity of some citizens.

\section{Conclusions}

The refugee crisis is a challenge that Europe and other political powers have to resolve. The response of Europe to this issue has not been effective. Scholars have analysed this situation (Jeandesboz \& Pallister-Wilkins, 2016; Pallister-Wilkins, 2016; Perkowski, 2016; Van Reekum, 2016) and they have remarked the humanitarian consequences of the refugee crisis and law implications of this phenomenon.

However, the critical reality has allowed for the outbreak of multiple caring initiatives with a high social impact (Flecha, Soler-Gallart \& Sordé, 2015). In this sense, the social impact of the action by Proactiva Open Arms is indisputable. Consequently, Proactiva Open Arms has been awarded different prizes. In addition, the Proactiva Open Arms case is a clear example of how solidarity with a high social impact drives a highly transformative process. Before the two Proactiva volunteers arrived in Lesbos, volunteers were leading different initiatives to help refugees but their social impacts were not so high. Nevertheless, the high number of lives which were saved by Proactiva Open Arms changed the responses of Frontex and the Greek Coastguard. Simultaneously, Proactiva Open Arms became a benchmark for other volunteers and NGOs on the island.

In parallel, the case of Proactiva Open Arms has allowed researchers to consider the concept of solidarity. Given that the term solidarity has been a versatile concept over the years and has been the subject of various reformulations both doctrinally and ideologically, the case of Proactiva Open Arms shows a new dimension of solidarity. When it is based on the defence of human rights, solidarity has a social impact and can transform political actions towards a solution to complex problems. Thus, the action of citizens such as the Proactiva Open Arms volunteers allows for a quicker response to 
social demands, producing empowerment of citizens that leads to community policy and influences institutions to change their actions or even forces them to act. In some situations, political authorities are slow due to bureaucratic procedures, complex partisan negotiations and regulations that hinder a rapid response to social problems. In this sense, citizen solidarity becomes a powerful alternative tool. Additionally, collaboration and dialogue between civil society and institutions is essential to manage this humanitarian crisis.

Finally, we must note that solidarity is usually under-examined when a conflict is analysed. However, the refugee crisis shows that solidarity movements that arise from people's empowerment also appear in critical situations. In future studies, it will be interesting to study the reason why some social movements have a high social impact and transform political actions, whereas others do not and why there are barriers within solidarity movements that could have negative results in the ever-increasing social impact of their intervention.

\section{Acknowledgements}

The research SOLIDUS leading to these results received funding from the H2020 Programme of the European Commission under Grant Agreement n 649489.

\section{References}

Arias, M., \& Palacios, S. (2016, September 26). 'Astral', un velero de lujo al rescate de refugiados. El Mundo. Retrieved from http://www.elmundo.es/internacional/2016/09/26/57e8024e22601d02 748b458f.html

Ataç, I., Rygiel, K., \& Stierl, M. (2016). Introduction: The Contentious Politics of Refugee and Migrant Protest and Solidarity Movements: Remaking Citizenship from the Margins. Citizenship Studies 20(5), 527-544. doi: 10.1080/13621025.2016.1182681

Clam Festival (2016). La oenegé proactiva open arms rep el premi pere casaldàliga a la solidaritat 2016. Retrieved from 
http://clamfestival.org/2016/05/la-oenege-proactiva-open-arms-rep-elpremi-pere-casaldaliga-a-la-solidaritat-2016/

Council of the European Union (2016). EU-Turkey Statement. 18 March, 2016. Press Release 144/16, 18/03/2106. Retrieved from http://www.consilium.europa.eu/press-releasespdf/2016/3/40802210113_en.pdf

Denzin, N.K. (1989). Interpretive biography. Thousand Oaks, CA: SAGE.

Denzin, N.K. (2006). Analytic autoethnography or Déjà Vu all over again. Journal of Contemporary Ethnography, 35, 419- 428. doi: 10.1177/0891241606286985

Denzin, N.K., \& Lincoln, Y.S. (Eds.). (2005). The SAGE handbook of qualitative research (3rd ed.). London, England: SAGE.

Diez, A., \& Fontseca, D. (2016). To Kyma. Rescat al mar Egeu [Video file]. Retrieved from http://www.ccma.cat/tv3/alacarta/sense-ficcio/tokyma-rescat-al-mar-egeu/video/5579941

El Periódico (2016, June 17). Proactiva Open Arms extiende su labor de rescate de refugiados al Mediterráneo central. El Periódico. Retrieved from http://www.elperiodico.com/es/noticias/internacional/proactivaopen-arms-extiende-labor-rescate-refugiados-mediterraneo-central5212805

European Commission (2016). Implementing the EU-Turkey Agreement Questions and Answers. European Commission Fact Sheet, MEMO 16/1221. Retrieved from http://europa.eu/rapid/press-release_MEMO16-1221_en.htm

European Parliament Affairs (2016, June 3). European Citizen's Prize: 50 people and organisations honoured. News European Parliament. Retrieved from http://www.europarl.europa.eu/resources/library/media/20160603RES 30254/20160603RES30254.pdf

Flecha, R., Soler-Gallart, M., \& Sordé, T. (2015) Social impact: Europe must fund social sciences. Nature, 528, 193. doi: 10.1038/528193d

Hein, J., \& Niazi, T. (2016). The primordial refugees: Religious traditions, global forced migration, and state-society relations. International Sociology, 31(6), 726-741. doi: 10.1177/0268580916662388 
International Organization for Migration (IOM) (2016). Missing Migrants Project Database. Retrieved from International Organization for

Migration website: http://missingmigrants.iom.int

Jeandesboz, J., \& Pallister-Wilkins, P. (2016). Crisis, Routine,

Consolidation: The Politics of the Mediterranean Migration Crisis.

Mediterranean Politics, 21(2), 316-320. doi:

10.1080/13629395.2016.1145825

Kousoulis, A.A., Ioakeim-Ioannidou, M., \& Economopoulos, K.P. (2016). Refugee crisis in Greece: not a one-country job. International Journal of Public Health, 67(1), 1-2. doi: 10.1007/s00038-016-0890-0

La Vanguardia (2016, November 16). Proactiva Open Arms recibe un premio por su labor de rescate marítimo. La Vanguardia. Retrieved from

http://www.lavanguardia.com/vida/20161116/411918050157/proactiv a-open-arms-recibe-un-premio-por-su-labor-de-rescate-maritimo.html

Mackey, R. (2015, October 29). As More Children Drown, Volunteers on Lesbos Say Rescues Are Left Largely to Them. New York Times. Retrieved from http://www.nytimes.com/2015/10/30/world/europe/asmore-children-drown-volunteers-on-greek-island-say-rescues-are-leftlargely-to-them.html?_r=0

Mautz, Ch. (2015). The Refugee in Europe. International and Multidisciplinary Journal of Social Sciences, 4(3), 293-311. doi: 10.17583/rimcis.2015.1803

Pallister-Wilkins, P. (2016). Interrogating the Mediterranean 'Migration Crisis'. Mediterranean Politics, 21(2), 311-315. doi: 10.1080/13629395.2016.1145826

Pérez, B. (2016, March 17). El socorrista de Badalona que salva vidas en el Egeo. $\quad$ El Periódico. $\quad$ Retrieved from http://www.elperiodico.com/es/noticias/sociedad/catala-de-any-oscarcamps-proactiva-open-arms-salva-vidas-refugiados-egeo-4954352

Perkowski, N. (2016). Deaths, Interventions, Humanitarianism and Human Rights in the Mediterranean 'Migration Crisis'. Mediterranean Politics, 21(2), 331-335. doi: 10.1080/13629395.2016.1145827

Proactiva Open Arms (2016). Proactiva Open Arms. Retrieved from https://www.proactivaopenarms.org 
Red Cross (2016). Crisis de refugiados en Europa. Informe situación $n^{\circ} 12$. Retrieved from Red Cross website: http://www.cruzroja.es/cre_web/principalci/assets/iscrisisrefugiados_1 2.pdf

Schoenbauer, R. (2016, October 7). Volunteers who saved lives on Lesvos nominated for Nobel Peace Prize. UNHCR News. Retrieved from http://www.unhcr.org/news/latest/2016/10/57f7732d4/volunteerssaved-lives-lesvos-nominated-nobel-peace-prize.html

Spanish Commission for Refugee Aid (2016). Lesbos, 'zona cero' del derecho de asilo. Retrieved from Spanish Commission for Refugee Aid website: http://www.cear.es/wpcontent/uploads/2016/04/Informe-Mision-Lesbos-marzo2016.pdf

UNHCR (2015). Lesvos island snapshot - 30 Dec 2015. Retrieved from UNHCR Data Portal website: http://reliefweb.int/report/greece/greece-lesvos-island-snapshot-30dec-2015

UNHCR (2016a). Syria Regional Refugee Response. Inter-agency Information Sharing Portal. Retrieved from UNHCR Data Portal website http://data.unhcr.org/syrianrefugees/regional.php

UNHCR (2016b). Refugees \& migrants sea arrivals in Europe. Monthly data update: December 2016. Retrieved from UNHCR Data Portal website http://reliefweb.int/sites/reliefweb.int/files/resources/Monthly_Arrival S_to_Greece_Italy_Spain_Jan_Dec_2016.pdf

Van Reekum, R. (2016). The Mediterranean: Migration Corridor, Border Spectacle, Ethical Landscape. Mediterranean Politics, 21(2), 336-441. doi: 10.1080/13629395.2016.1145828

Gemma Álvarez-Jiménez is PhD candidate in Political Science at the Department of Sociology at the University of Barcelona, Spain

Maria Padrós-Cuxart is associate professor in the Department of Didactics and Educational Organization at the University of Barcelona, Spain

Contact address: Diagonal Nord, Facultat d'Economia i Empresa (Edifici 690). Diagonal 690, 08034, Barcelona. Spain. Email: gemma.alvarez@ub.edu 


\section{RIMCIS Social Sciences}

\section{Hipatia Press}

www.hipatiapress.com

Instructions for authors, subscriptions and further details:

http://rimcis.hipatiapress.com

\section{Cuerpos Aliados y Lucha Política: Hacia una Teoría Performativa de la Asamblea}

Teresa Plaja-Viñas ${ }^{1}$

1) Universidad de Barcelona. España

Date of publication: July $30^{\text {th }}, 2017$

Edition period: July 2017 - November 2017

To cite this article: Plaja-Viñas, T. (2017). Cuerpos Aliados y Lucha Política: Hacia una Teoría Performativa de la Asamblea [Review of the book]. International and Multidisciplinary Journal of Social Sciences, 6(2), 230-231. doi: 10.17583/rimcis.2017.2823

To link this article: http://doi.org/10.17583/rimcis.2017.2823

\section{PLEASE SCROLL DOWN FOR ARTICLE}

The terms and conditions of use are related to the Open Journal System and to Creative Commons Attribution License (CC-BY). 


\section{Review}

Butler, J. (2017). Cuerpos aliados y lucha política. Hacia una teoría performativa de la asamblea (1 $1^{\mathrm{a}}$ edición). Barcelona: Paidós Básica. ISBN 978-84-493-3303-3

Judith Butler es una de las autoras más reconocidas dentro del feminismo por su desarrollo de la Teoría Queer. Con el libro El Género en disputa. Feminismo y la subversión de la identidad (1990), Butler presentó su teoría sobre la performatividad del género, ampliando el análisis en obras posteriores. Dicha perspectiva abrió la puerta a una reducción de la presión ejercida por las normas de género, con el fin de posibilitar, lo que la autora define como, una vida más vivible. Durante su carrera académica, Butler no solo ha trabajado temas de género, sino que ha ampliado sus análisis a cuestiones éticas, de filosofía política o de derechos humanos.

En el libro Cuerpos aliados y lucha política: Hacia una teoría performativa de la asamblea, Butler desarrolla su teoría de la performatividad incluyendo lo que Arendt definía como "acciones concertadas". Para ello, la autora realiza un análisis normativo de la realidad a partir de los movimientos sociales que han tenido lugar en todo el mundo en los últimos años, como Occupy Wall Street, la ocupación de la plaza Tahrir o el movimiento Black Lives Matter.

A lo largo de los seis capítulos que constituyen el libro, la autora incluye los actos corporales dentro de la teoría de la performatividad. Para dicho estudio, Butler establece un diálogo con las aportaciones de autores como Hannah Arendt o Theodor Adorno. 
En el primer capítulo, la autora parte de las acciones performativas individuales para presentar la asamblea como un "ejercicio performativo de su derecho a la aparición, es decir, una reivindicación corporeizada de una vida más vivible" (Butler, 2017, p. 31). A continuación, expone como en las movilizaciones en las que se reclama un espacio público existen elementos comunes, en ellas, las personas excluidas y que viven situaciones de precariedad se unen y se movilizan. En el tercer capítulo, centra su análisis en cómo, aunque los acontecimientos son locales, existe una reversibilidad de lo próximo y lo distante en las exigencias éticas. En el siguiente capítulo, Butler realiza un análisis de la vulnerabilidad a partir del estudio y la vinculación de la vulnerabilidad del cuerpo, las alianzas con otras personas y la política de la calle. En el capítulo cinco, la autora debate sobre qué individuos configuran el "pueblo" y cómo se manifiesta. En este debate, Butler entiende estas acciones de reclamo del espacio público como actos performativos. Finalmente, Judith Butler trabaja en torno a la pregunta " ¿se puede llevar una buena vida en medio de una mala vida?” (Butler, 2017, p. 195) vinculándola con la participación política, la vulnerabilidad y la interdependencia entre las personas para llevar una buena vida.

El libro nos permite reflexionar sobre la soberanía popular y los movimientos sociales, desde una nueva perspectiva teórica. Dicha reflexión resulta de interés para ámbitos académicos, como la ciencia política o la sociología, por ejemplo, y a la vez para aquellos actores envueltos en acciones sociales.

\section{Referencias}

Butler, J. (1990). El género en disputa. El feminismo y la subversión de la identidad. Barcelona: Paidós.

Butler, J. (2017). Cuerpos aliados y lucha política. Hacia una teoría performativa de la asamblea ( $1^{\mathrm{a}}$ edición). Barcelona: Paidós Básica

Teresa Plaja Viñas, Universidad de Barcelona teresa.plaja@ub.edu 